\title{
ARITHMETIC ON SINGULAR DEL PEZZO SURFACES
}

\author{
D. F. CORAY and M. A. TSFASMAN
}

[Received 24 April 1986—Revised 8 April 1987]

To Yu. I. Manin on his fiftieth birthday

\section{Introduction}

The study of singular cubic surfaces is quite an old subject, since their classification (over $\mathbb{C}$ ) goes back to Schläfli [39] and Cayley [8]. However, a recent account by Bruce and Wall [6] has shown that modern singularity theory can give much insight into this classification. One of the main themes of the present paper is that this approach is also useful over an arbitrary perfect field $k$ for studying the $k$-birational properties of singular cubic surfaces, and of certain other singular surfaces which are defined below.

Recall that an absolutely irreducible algebraic variety $V$, defined over $k$, is said to be $k$-rational (respectively, $k$-unirational) if its function field $k(V)$ is (respectively, is contained in) a purely transcendental extension of $k$. Throughout this paper $\bar{k}$ denotes an algebraic closure of $k$, and $\bar{V}=V \times{ }_{k} \bar{k}$. We say that $V$ is rational if $\bar{V}$ is $\bar{k}$-rational, and we write $\mathbb{P}^{n}$ for $\mathbb{P}_{\bar{k}}^{n}$. Unless stated otherwise, the notation $V \subset \mathbb{P}_{k}^{n}$ implies that $V$ is a projective subvariety of $\mathbb{P}_{k}^{n}$, defined over $k$. In Part I we prove:

THEOREM A. Let $V \subset \mathbb{P}_{k}^{3}$ be a singular cubic surface. Suppose $\bar{V}$ has precisely $\delta$ isolated double points. Then $\delta \leqslant 4$ and $V$ is birationally equivalent (over $k$ ) to:

$\mathbb{P}_{k}^{2}$ if $\delta=1$ or 4 ;

a smooth Del Pezzo surface of degree 4 with a k-point if $\delta=2$;

a smooth Del Pezzo surface of degree 6 if $\delta=3$.

In particular, $V$ is $k$-unirational if $V(k) \neq \varnothing$.

Recall that a smooth Del Pezzo surface is a smooth projective surface $V$ whose anticanonical class is ample. Many arithmetic properties of these surfaces were investigated by Manin (cf. [32]).

Theorem A is proved in $\S 1$ with the methods of modern singularity theory (but with a restriction on the characteristic of $k$ ). The most interesting feature of this approach is that it provides a unified treatment of the two cases $\delta=2$ and $\delta=3$. Actually, the theorem was already known for $\delta=1$ or 4 (cf. [40]), and for $\delta=3$ (cf. [17]). In all these cases one can produce an explicit birational equivalence, and the proof (which does not depend on a preliminary classification of all possible singularities) is valid in arbitrary characteristic. In $\S 2$ we give a similar argument for the case where $\delta=2$. Although the presentation is completely independent of $\S 1$, one must emphasize that the choice of the Cremona transformation to be applied was suggested by the results of that section. Indeed, one had to look for a transformation whose effect on $V$ was as described in $\S 1$. 
In fact, Theorem 2.2 is a more precise version of Theorem $\mathrm{A}$. The reason is that the treatment of $\S 2$ not only yields explicit equations for the smooth model of $V$, but also gives some further information on the transform (the action of $\operatorname{Gal}(\bar{k} / k)$ over a particular set of lines, for instance). Thus we come to another theme of this paper, which gives various applications and many examples of concrete computations. We cannot describe them all in this introduction; the reader is invited to browse through $\S \S 3$ and 4 . Particularly representative are Example 3.3 (and the subsequent Remark, which is used again in $\$ \S 4$ and 7), Proposition 3.4 (with its application to the Zariski Problem), Proposition 4.2 (showing that the situation is not trivial even over a finite field), Proposition 4.6 (which is related to the computation of the Chow group of a Châtelet surface (Proposition 4.7)), and Corollary 4.9. See also Proposition 7.6.

The reader can also refer to the survey [33], which, for technical reasons, appeared before the present paper, but includes many of its results (without proof) and puts them into perspective (see especially $\$ \S 2$ and 7). Before proceeding, we must recall some results and definitions, which will be used freely in what follows.

Proposition 0.1 (Artin). Let $V^{\prime}$ be a normal, complete algebraic surface over an algebraically closed field $k$. Let $E=\sum E_{i}$ be a connected curve on $V^{\prime}$, with irreducible components $E_{i}$, and assume $V^{\prime}$ is non-singular along $E$. Then the following conditions are equivalent.

(a) The intersection matrix $\left\|\left(E_{i} . E_{j}\right)\right\|$ is negative definite, and every component $E_{i}$ of $E$ is a smooth rational curve with self-intersection $\left(E_{i}^{2}\right)=-2$.

(b) $E$ is contractible, i.e. there exists a morphism $f: V^{\prime} \rightarrow V$ which is an isomorphism at every point of $V^{\prime} \backslash E$ and maps the curve $E$ into a normal point $P$ of the surface $V$. Moreover, any canonical divisor $K_{V}$ of $V$ is locally principal at $P$, and the total transform $K_{V^{\prime}}=f^{*}\left(K_{V}\right)$ is a canonical divisor on $V^{\prime}$.

Furthermore, if (a) or (b) holds, then the morphism $f$ preserves the Euler characteristic: $\chi\left(V^{\prime}\right)=\chi(V)$.

This is Theorem 2.7 of [1]. In this situation, $P$ is called a rational double point. (By definition (see [2]), $\chi\left(V^{\prime}\right)=\chi(V)$ means that $P$ is a rational singularity. Now it follows from (a) that $\left(E_{i} . K_{V^{\prime}}\right)=0$; so the 'fundamental cycle' of $E$ (as defined in [2]), $Z=\sum r_{i} E_{i}$, verifies that $\left(Z . K_{V^{\prime}}\right)=0$. Hence $\left(Z^{2}\right)=-2$ and $P$ is double, by Corollary 6 of [2]. With the results of [2] this argument can be done backwards to show that, conversely, a rational double point verifies Condition (a).)

If $V^{\prime}$ is smooth then $f: V^{\prime} \rightarrow V$ is the minimal desingularization of $V$ (see [30, Corollary 27.3]). Finally, if a normal surface $V$ has only rational double points, there is a Dynkin diagram $\Delta$ associated with its minimal desingularization. The vertices, of weight -2 , correspond to the $E_{i}$. Two vertices are joined by an edge if and only if the corresponding components $E_{i}, E_{j}$ meet, in which case $\left(E_{i}, E_{j}\right)=1$. Of course, the number of connected components of $\Delta$ is equal to the number of singularities of $V$. We shall say that $V$ has singularities of type $\Delta$.

Proposimion 0.2 (Bruce and Wall [6]). Let $V \subset \mathbb{P}_{\mathbb{C}}^{3}$ be an irreducible cubic surface having only isolated singularities. Then either

(a) $V$ is a cone over a smooth plane cubic, the vertex being a simple elliptic 
singularity of type $\tilde{E}_{6}$,

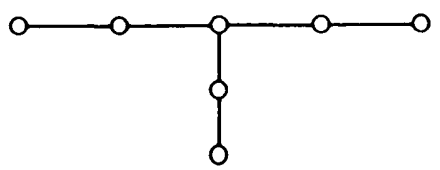

or

(b) V has only rational double points, and their type is a proper subgraph of the extended Dynkin diagram $\tilde{E}_{6}$.

See [6] for the list of all possibilities and the number of distinct lines on $V$ in each case. We have not defined the type of an elliptic singularity, for which we refer to [31, Chapter 1, Example 5, and Chapter 7, no. 23]. Indeed only Case (b) is of interest for our purposes. But Case (a) completes the picture nicely (cf. $[37,34])$.

In Part II we use the analogous result for the intersections of two quadrics:

Proposition 0.3 (Pinkham [37], Knörrer [26], Wall [47]). Let $V \subset \mathbb{P}_{\mathbb{C}}^{4}$ be an irreducible intersection of two quadrics which has only isolated singularities. Then either

(a) $V$ is a cone over a smooth quartic of genus 1 in $\mathbb{P}_{C}^{3}$, the vertex being a simple elliptic singularity of type $\tilde{D}_{5}$,

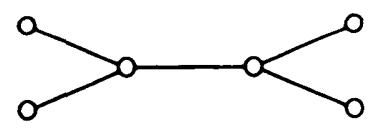

or

(b) V has only rational double points, and their type is a proper subgraph of the extended Dynkin diagram $\bar{D}_{5}$.

Actually, for our arithmetic applications we shall need a slightly more precise version of this proposition. Indeed, that the singularities are rational in Case (b) follows easily from Proposition 0.2. Then one can use the results of Demazure [19] to classify all possible Dynkin diagrams up to the action of the appropriate Weyl group (Proposition 5.6). This approach goes back to Du Val [20] and Coxeter [18], and the classification is valid over a field of arbitrary characteristic. A brief sketch of this argument is included in $\S 5$ to help the unfamiliar reader, and also to fix notation. A remarkable feature of the classification, which was already noticed by $\mathrm{Du} \mathrm{Val}$, is that there are two distinct equivalence classes of subdiagrams of type $2 A_{1}$ (and the same thing happens for type $A_{3}$ ). In one case, the line joining the two double points lies on the surface; in the other case, it does not. This situation does not arise with cubic surfaces, which have only one class for each type. Moreover, the distinction between the two classes has arithmetic significance. In $\S 7$ a surface $V \subset \mathbb{P}_{k}^{4}$ of the second kind is called an Iskovskih surface when the two double points are conjugate. Using the classification, we show that, in some sense, the only arithmetically interesting objects are the Iskovskih surfaces (Theorem 7.2):

THEOREM B. Let $V \subset \mathbb{P}_{k}^{4}$ be a singular irreducible intersection of two quadrics which has only rational double points. Suppose $V$ is not an Iskovskih surface. 
Then the following assertions hold:

(TR) if $V$ contains a smooth $k$-point, then $V$ is $k$-rational;

(HP) if $k$ is a number field and $V$ contains a smooth $k_{v}$-point, for every completion $k_{v}$ of $k$, then every proper model of $V$ contains a $k$-point.

Neither assertion holds for the class of Iskovskih surfaces; but it is true that if $V$ is an Iskouskih surface and $V(k) \neq \varnothing$, then $V$ is $k$-unirational.

Here we may point out an error in a widely quoted paper of Nagata [35]: Proposition 10, on p. 368, simply denies the existence of Iskovskih surfaces! (It asserts, as a special case, that the line joining two double points of an intersection of two quadrics in $\mathbb{P}^{4}$ lies on the variety.)

When we analyse the arguments of Part II, we see that the essential ingredients are certain results of Demazure, which hold for more general surfaces. Since it is well known that the theory of smooth Del Pezzo surfaces becomes simpler as the degree increases, it is natural to adopt this more general point of view in our study.

Definition. A generalized Del Pezzo surface is a smooth projective surface $V$ which is 'of negative type' in the sense of Demazure, that is, $\left(K_{V}^{2}\right)>0$ and $\left(D . K_{V}\right) \leqslant 0$ for all effective divisors $D$ on $V$. Its degree is the number $d=\left(K_{V}^{2}\right)$.

Note that this definition is independent of the field of definition. It is easy to see that it includes the usual Del Pezzo surfaces.

Proposition 0.4 (Demazure). The surface $V$ is a generalized Del Pezzo surface (of degree $d$ ) if and only if $\bar{V}$ is isomorphic to one of the following rational varieties:

$\mathbb{P}^{1} \times \mathbb{P}^{1}$ (of degree 8 );

the ruled surface $F_{2}$ over $\mathbb{P}^{1}$ (of degree 8 );

the surface $\mathbb{P}^{2}(\Sigma)$ obtained from $\mathbb{P}^{2}$ by blowing up a set $\Sigma$ of $r$ points in 'almost general position'; then $0 \leqslant r \leqslant 8$ and $d=9-r$.

The points blown up can be infinitely near. More precisely, starting from $X_{0}=\mathbb{P}^{2}$, one constructs a sequence of surfaces

$$
\mathbb{P}^{2}=X_{0} \leftarrow X_{1} \leftarrow \ldots \leftarrow X_{r}=\mathbb{P}^{2}(\Sigma),
$$

where each $X_{i}$ is obtained by blowing up one point $x_{i} \in X_{i-1}$. The set $\Sigma=$ $\left\{x_{1}, \ldots, x_{r}\right\}$ is in almost general position if and only if, at each stage, $x_{i}$ does not belong to any irreducible curve of $X_{i-1}$ with self-intersection -2 .

Proof. The proof is in [19], except for a few details. One has to show that a generalized Del Pezzo surface $V$ is rational, and this follows from the general theory of surfaces. Namely, all the plurigenera are equal to zero. Indeed, suppose there were an effective divisor $D$ in the class $m K_{V}$; then $\left(D . K_{V}\right)=m\left(K_{V}^{2}\right)>0$, a contradiction. Now, by Proposition 2.4 (p. 378) of [4], $p_{g}=0$ and $\left(K_{V}^{2}\right)>0$ imply $q=0$. Thus $V$ is rational, by Castelnuovo's criterion [4, p. 381, Theorem 5.1], since $P_{2}=q=0$.

This result enables one to apply Proposition III.3 of [19] (where $V$ was assumed to be rational). Actually, the ruled surface $F_{2}$ is not mentioned by Demazure, but 
this is due to an oversight immediately before the statement of Proposition III.3: in (d), replace ' $n>1$ ' by ' $n>2$ ', since $F_{2}$ is also of negative type.

Finally, to show that all the surfaces listed are generalized Del Pezzo surfaces one can refer to Theorem III.1 of [19]. An easy verification shows that a set $\Sigma$ as described in Proposition 0.4 is in almost general position in the sense of the definition of [19, Part III, §2].

ReMARK. Let $D$ be an irreducible curve on $\bar{V}$ with negative self-intersection. Then either $\left(D^{2}\right)=\left(D . K_{V}\right)=-1$, or $\left(D^{2}\right)=-2$ and $\left(D . K_{V}\right)=0$, since $p_{a}(D) \geqslant 0$. In the former case, $D$ is an exceptional divisor of the first kind; for reasons that will appear later, we shall say that $D$ is a line of $\bar{V}$. In the latter case, $D$ will be called an irreducible root of $\bar{V}$. For instance, the condition for 'almost general position' in Proposition 0.4 can be expressed quite simply: 'Never blow up a point lying on an irreducible root!' (... for the obvious reason that the proper transform of the root would be an irreducible curve with self-intersection less than -2).

EXAMPLE 0.5. Let $V \subset \mathbb{P}_{\mathbb{C}}^{3}$ be an irreducible cubic surface having only isolated singularities, and suppose $V$ is not a cone. Let $f: V^{\prime} \rightarrow V$ be its minimal desingularization. Then $V^{\prime}$ is a generalized Del Pezzo surface of degree 3. Its lines are the proper transforms of the lines of $V$. Its irreducible roots are the irreducible components of the curves on $V^{\prime}$ which are contracted by $f$ into the singular points of $V$. Thus they correspond to the vertices of the Dynkin diagram associated with the singularities of $V$.

Proof. By Proposition $0.2, V$ has only rational double points. By Proposition $0.1(\mathrm{~b}), K_{V^{\prime}}=f^{*}\left(K_{V}\right)$. Now we know that $-K_{V}$ is in the class of the hyperplane sections. Hence $\left(K_{V^{\prime}}^{2}\right)=3$ and $V^{\prime}$ is of negative type. Moreover, the lines of $V$ are the only rational curves $L$ such that $\left(L . K_{V}\right)=-1$. Finally, the irreducible roots $D$ verify the condition $\left(D . K_{V^{\prime}}\right)=0$; so they are concentrated above the singularities of $V$ (of type $\Delta$, say). By Proposition 0.1(a), they are precisely all the irreducible components $E_{i}$, that is, they correspond exactly to the vertices of $\Delta$.

This example has the following extension to generalized Del Pezzo surfaces:

Proposition 0.6 (Demazure). Let $V^{\prime}$ be a generalized Del Pezzo surface of degree $d$ over an algebraically closed field $k$. Let $f_{0}: V^{\prime} \rightarrow V_{0}$ be the rational map defined by the complete linear system $\left|-i K_{V^{\prime}}\right|$, where

$$
i= \begin{cases}1 & \text { if } d \geqslant 3, \\ 2 & \text { if } d=2, \\ 3 & \text { if } d=1 .\end{cases}
$$

Then $f_{0}$ is a morphism which contracts all the irreducible roots of $V^{\prime}$ and is an isomorphism everywhere else. Its image $V_{0}$ is normal, with only rational double points. For $d \geqslant 3, V_{0}$ is anticanonically embedded as a surface of degree $d$ in $\mathbb{P}^{d}$.

This is proved in [19, Part V, Theorems 1 and 2, Corollary 3].

Definition. A singular Del Pezzo surface (of degree $d$ ) is a singular normal projective surface $V$ which is obtained from a generalized Del Pezzo surface $V^{\prime}$ 
(of the same degree) by contracting all the irreducible roots (and nothing else; cf. Proposition 0.1(b)). Equivalently, for $d \geqslant 3, V$ is a singular surface which is isomorphic to the anticanonical image of a generalized Del Pezzo surface $V^{\prime}$.

This definition makes sense. Indeed, by Proposition 0.6 there exists such a contraction $f_{0}: V^{\prime} \rightarrow V_{0}$. But if we choose any other contraction (say $f: V^{\prime} \rightarrow V$ ), following the prescriptions of Proposition 0.1(b), then the birational map $f \circ f_{0}^{-1}$ is a morphism, by [19, Corollary 1 to Theorem V.2], and hence an isomorphism, as follows from the Zariski Main Theorem (since $V$ is normal).

Example 0.7. (a) Every singular Del Pezzo surface $V$ of degree 3 (respectively 4) embeds anticanonically as a cubic surface in $\mathbb{P}_{k}^{3}$ (respectively as the intersection of two quadrics in $\mathbb{P}_{k}^{4}$ ).

(b) Conversely, every singular cubic surface $V \subset \mathbb{P}_{k}^{3}$ (respectively every singular intersection of two quadrics $\left.V \subset \mathbb{P}_{k}^{4}\right)$ with only rational double points, is a singular Del Pezzo surface of degree 3 (respectively 4).

Proof. (a) By Proposition 0.6, $V$ embeds anticanonically as a surface of degree $d$ in $\mathbb{P}_{k}^{d}$. For $d=3$, there is nothing more to prove. For $d=4$, it follows from [19, Corollary 3 to Theorem V.2] that $h^{0}\left(V, \omega_{V}^{-2}\right)=13$. Hence $V \subset \mathbb{P}_{k}^{4}$ is contained in a pencil of quadrics. (Indeed the family of all quadrics in $\mathbb{P}_{k}^{4}$ has dimension 14 and, by the above, they only cut out on $V$ a linear system of dimension 12.) Since $V$ has degree 4, it follows that $V \subset \mathbb{P}_{k}^{4}$ is the intersection of two quadrics.

(b) Consider the minimal desingularization $f: V^{\prime} \rightarrow V$, and repeat the argument of Example 0.5.

In Example 0.7(b), it is probably sufficient to assume that $V$ has only isolated singularities and is not a cone. Indeed this is true at least if $k \subset \mathbb{C}$, as follows from Proposition 0.2 (respectively Proposition 0.3 ). But we have no proof in the general case, in particular for very small characteristics.

In Part III we study the singular Del Pezzo surfaces of degree $d \geqslant 5$. Note that $d$ is then at most equal to 8 . The following fact goes back to Du Val [20]:

Proposition 0.8. Let $V$ be a singular Del Pezzo surface of degree $d \geqslant 5$. Then $\bar{V}$ has only rational double points, and their type is a subgraph of the Dynkin diagram:

$$
\begin{array}{ll}
A_{4} & \text { if } d=5 \\
A_{1} \times A_{2} & \text { if } d=6 ; \\
A_{1} & \text { if } d=7 \text { or } 8
\end{array}
$$

In $\S 8$ we make a list of all possibilities, including diagrams of irreducible roots and lines. The diagrams we obtain are equivalent to the dual pictures of Timms [44], but they are slightly more precise. We use them to show that the singular Del Pezzo surfaces of degree $d \geqslant 5$ have arithmetic properties quite similar to those of the smooth Del Pezzo surfaces of the same degree (Theorem 9.1):

Theorem C. Let $V$ be a singular Del Pezzo surface of degree $d \geqslant 5$. Then: for $d=5$ or $7, V$ is $k$-rational; for $d=6$ or 8 , both assertions (TR) and (HP) of Theorem B hold for V. 
Not surprisingly, the classification of these surfaces gets simpler and simpler as the degree increases. So these varieties can be used as tools for proving theorems about the surfaces of smaller degree. Nevertheless we deal with them only in Part III because, unlike the surfaces of degree 3 or 4 , they do not have an obvious interpretation in terms of equations. Thus Parts II and III are almost independent of Part I, if we except some occasional reference to provide an alternative argument. This enables us to illustrate several different techniques.

Last but not least, it is our great pleasure to thank J.-L. Colliot-Thélène, B. È. Kunjavskiî, Yu. I. Manin, A. N. Skorobogatov, and Sir Peter Swinnerton-Dyer for their constant encouragement and their many valuable comments and suggestions. In addition, the proof of Proposition 4.2 is due to Swinnerton-Dyer, and Proposition 4.7 was communicated by Colliot-Thélène. The referee's remarks have helped to clarify the exposition.

\section{Part I. Cubic surfaces}

\section{Birational classification of singular cubics}

Throughout this paper, we shall suppose that the field $k$ is perfect. One reason for this assumption will be seen in Remark (a) after Lemma 1.1. Finite fields are allowed, but a constant assumption will be that $k$ has more than five elements. In fact, the proof of Lemma 1.2 is not applicable to the Châtelet surface $y^{2}+z^{2}=x^{3}-x$ over $k=\mathbb{F}_{3}$, though Colliot-Thélène has shown by another method that this surface is $k$-unirational.

(Sketch of proof. Let $T$ be defined by $\left\{u_{i}^{2}+v_{i}^{2}=x-i\right\}_{i=0}^{2}$. On setting $y+(\sqrt{ }-1) z=\prod\left(u_{i}+(\sqrt{ }-1) v_{i}\right)$, we obtain a dominant map from $T$ to the Châtelet surface. Now $T$, being birationally equivalent to an intersection of two quadrics which contains a line, is $k$-rational (cf. [15, Chapter II, Proposition 2]).)

To begin with, we recall the statement of Nishimura's lemma, which is used at several places in this paper:

Lemma 1.0 ('Nishimura's lemma'). Let $f: X \ldots \rightarrow Y$ be a $k$-rational map from an integral $k$-variety $X$ to a proper $k$-variety $Y$. Suppose $X(k)$ contains a regular $k$-point. Then $Y(k) \neq \varnothing$.

For a proof and further reference, see [12, Lemma 3.1.1].

LEMMA 1.1 (cf. [40]). Let $V \subset \mathbb{P}_{k}^{3}$ be a singular cubic surface with only isolated singularities. Suppose $V$ is not a cone, and let $\delta$ be the number of double points of $\bar{V}$. Then $\delta \leqslant 4$; and $V$ is $k$-rational in each of the following cases:

(i) if $V$ has a double point defined over $k$;

(ii) if $\delta=1$ or 4 ;

(iii) if $\delta=3$ and $V(k) \neq \varnothing$.

Proof. Since $\bar{V}$ is not a cone, it has only double points for singularities. If one of them is defined over $k$, then projecting from this double point maps $V$ birationally into a plane. This deals with (i), and also with the case where $\delta=1$, since $k$ is perfect. 
Now suppose $\delta \geqslant 3$. We observe that no three double points are collinear. Otherwise a general plane through them would meet the surface $\bar{V}$ in the cycle $2 L+L^{\prime}$, where $L$ is the line joining the points, and $L^{\prime}$ is another line. So the whole line $L$ would be double on $\bar{V}$, but by assumption $V$ has only isolated singularities. Then one sees easily that no four double points of $\bar{V}$ lie in a plane.

Now recall (cf. [17, p. 57]) that four non-coplanar points $Q_{1}, \ldots, Q_{4}$ of $\mathbb{P}^{3}$ define a Cremona transformation $\Theta=T_{33}^{\text {tet }}$, whose associated linear system consists of all cubic surfaces on which each $Q_{i}$ is at least double. (With a suitable choice of coordinates, this is simply the map $(x, y, z, t) \mapsto(1 / x, 1 / y, 1 / z, 1 / t)$.) This has the following consequences.

(1) If $\delta=4$, the Cremona transformation $\Theta$ defined by the four double points maps $V$ into a plane. This completes the proof in Case (ii).

(2) If $\delta=3$ and $V(k) \neq \varnothing$, let $Q_{1}, Q_{2}, Q_{3}$ be the three double points and $Q_{4} \in V(k)$. Since we have already dealt with Case (i), we can assume that none of the double points is defined over $k$. Then $Q_{4}$ does not lie in the plane spanned by $Q_{1}, Q_{2}$, and $Q_{3}$. (Indeed the intersection of this plane with $\bar{V}$ consists of three lines, on which the group $\operatorname{Gal}(\bar{k} / k)$ acts transitively.) Hence $Q_{1}, \ldots, Q_{4}$ define a Cremona transformation $\Theta$, which maps $V$ into a quadric $W$. To prove (iii), it suffices to see that $W$ contains a smooth $k$-point.

Now $Q_{4} \in V(k)$ is smooth; hence it follows from Nishimura's lemma (Lemma $1.0)$ that $W(k) \neq \varnothing$. If $W$ is smooth, we have finished. Hence we shall assume that $W$ is a quadratic cone. If $k$ is finite, we can find a smooth $k$-point on a plane section of $W$, since $k$ is a $C_{1}$ field! Thus we may assume that $k$ is infinite. Let $U \subset V$ be an open subset on which $\Theta$ restricts to an isomorphism. Then we can find a line $L \subset \mathbb{P}_{k}^{3}$ whose intersection with $V$ is contained in $U$. The image $\Theta(L \cap V)$ is a 0 -cycle of degree 3 on $W$ and consists of smooth points. Thus the result follows from an elementary argument of descent on the degree (also used in [17, proof of Lemma 3]).

(3) Finally, $\delta$ cannot exceed 4 . Indeed suppose $Q_{1}, \ldots, Q_{5}$ are double points of $\bar{V}$, and let $Q_{6}$ be a general point of $\mathbb{P}^{3}$ (in particular, not lying on $\bar{V}$ ). Then the Cremona transformation $\Theta$ defined by $Q_{1}, \ldots, Q_{4}$ has the property that the line through $\Theta\left(Q_{5}\right)$ and $\Theta\left(Q_{6}\right)$ is the image of a twisted cubic $\Gamma$ through $Q_{1}, \ldots, Q_{6}$. This is absurd: indeed $\Gamma \notin \bar{V}$, since $\Gamma \ni Q_{6}$; whence we get a contradiction to Bézout's theorem.

Remarks. (a) The lemma does not hold over an imperfect field, even in the case where $\delta=1$. As an example we may take the surface $V$ defined over the power-series field $k=\mathbb{F}_{3}((\lambda))$ by the equation

$$
x^{3}+\lambda\left(y^{3}-y z^{2}+z^{3}\right)+\lambda^{2}\left(y^{2} t-t^{3}\right)=0 .
$$

A $\lambda$-adic argument shows that $V(k)=\varnothing$. Now, one checks easily that $\bar{V}$ has only one double point, with coordinates $\left(\lambda^{\frac{2}{3}}, 0,0,1\right)$.

(b) For $\delta=3$ the lemma does not hold without the assumption that $V(k) \neq \varnothing$. For instance, let $k=\mathbb{Q}_{p}$ and $\theta$ be a root of a polynomial of degree 3 which is irreducible modulo $p$. Then the equation $p t^{3}=N_{k(\theta) / k}\left(x+\theta y+\theta^{2} z\right)$ defines a surface $V$ such that $\delta=3$ and $V(k)=\varnothing$.

(c) Another argument has been given in [45] for the case where $\delta=4$ : suppose it is known that $\bar{V}$ has only rational double points (cf. Proposition 0.2); then, on the minimal desingularization of $V$, one can blow down a set of six exceptional 
divisors of the first kind (corresponding to the edges of the tetrahedron through the nodes) and obtain a Del Pezzo surface $W$ of degree 9 (cf. the proof of Theorem 7.2). Since $W$ is a Severi-Brauer variety and contains a 0 -cycle of degree $1, W$ is isomorphic to $\mathbb{P}_{k}^{2}$. ( $W$ contains an effective 0 -cycle of degree 3 because $V$ is a cubic surface, and an effective 0 -cycle of degree prime to 3 , for instance because we can apply Case (i) over a field of definition of any one of the double points.)

For $\delta=2$, one has only the following weaker result. Indeed, the surface $V_{3}$ of Example 3.3 is not $\mathbb{Q}$-rational.

Lemma 1.2. Let $V \subset \mathbb{P}_{k}^{3}$ be a cubic surface having two conjugate double points $Q_{1}, Q_{2}$, and no other singularities. Then $V$ is $k$-unirational.

Proof. Of course $V(k) \neq \varnothing$, since the line $L_{0}$ through $Q_{1}$ and $Q_{2}$ is contained in $V$. By the argument given in [32, Chapter IV, Theorem 7.8] (cf. also [32, Chapter II, Theorem 2.9]), the lemma will be proved if we show that $V$ contains a $k$-point that does not lie on any line of $\bar{V}$.

First we deal with the case of a finite field. As $\operatorname{card}(k) \geqslant 5$, we can find a plane $\pi \subset \mathbb{P}_{k}^{3}$ through $L_{0}$, such that $\pi \cap V=L_{0} \cup C_{0}$, where $C_{0}$ is a smooth conic. Now $k$ is a $C_{1}$ field, and hence $C_{0}(k) \neq \varnothing$. Then $C_{0}$, being isomorphic to $\mathbb{P}_{k}^{1}$, contains a $k$-point which does not lie on any line of $\bar{V}$. Indeed, by assumption, $\operatorname{card}(k)>5$, and one can show (cf. Fig. 3 in $\S 3$ ) that no more than six lines can possibly meet $C_{0}$ in a $k$-point.

Thus we may assume that $k$ is infinite. It is tempting to try and prove the lemma by considering the tangent plane $T_{0}$ to $V$ at a general $k$-point $P_{0}$ of $L_{0}$, and hoping that $T_{0} \cap V$ is of the form $L_{0} \cup C_{0}$ with $C_{0}$ a smooth conic through $P_{0}$. However this idea does not work, because $T_{0} \cap V$ is singular at $Q_{1}, Q_{2}$, and $P_{0}$. Hence the intersection cycle $T_{0} . V$ is of the form $2 L_{0}+L$, where the line $L$ may or may not coincide with $L_{0}$. It follows readily that all smooth points on $L_{0}$ have one and the same tangent plane $T_{0}$. Moreover we see that, in the case where $L=L_{0}$, we do not get any new $k$-point from this construction. The argument that follows is inspired by a technique used by Skolem in [41, pp. 308-309]; cf. [17, footnote ${ }^{2}$.

First we consider the case in which $L \neq L_{0}$. (This happens if and only if $V$ has singularities of type $2 A_{1}$.) Pick a general $k$-point $P$ on $L$, and let $\pi$ be the tangent plane to $V$ at $P$. Then $\pi \neq T_{0}$, because $L$ is simple in the intersection $T_{0} . V$. Further, the points on $L$ do not all have the same tangent plane $\pi$, for at the $k$-point $L_{0} \cap L$ (which is non-singular, because the $Q_{i}$ are conjugate), we know that the tangent plane is $T_{0}$. Therefore $\pi \cap V$ is of the form $L \cup C$, where $C$ is a smooth conic through $P$. As $k$ is infinite, it follows that $C$ contains a $k$-point that does not lie on any line of $\bar{V}$.

Next we suppose that $L=L_{0}$, that is, $T_{0} . V=3 L_{0}$. (This happens whenever $V$ has singularities of type $2 A_{2}$.). Then we proceed in two steps:

(i) Let $K$ be the quadratic extension of $k$ over which $Q_{1}$ is defined. Let $\pi_{1}$ be a plane through $L_{0}$ defined over $K$, but not over $k$, and such that $\pi_{1} \cap V_{K}$ is of the form $L_{0} \cup C_{1}$, where $C_{1}$ is a smooth conic, defined over $K$. We denote by $C_{2}$ its conjugate. As $Q_{1} \in C_{1}(K)$, the curve $C_{1}$ contains infinitely many $K$-points. If $P_{1}$ is such a point, other than $Q_{1}$ or $Q_{2}$, then its conjugate $P_{2}$ lies on $C_{2} \backslash C_{1}$, and the line $L$ joining $P_{1}$ and $P_{2}$ meets $V$ in a $k$-point $P \notin L_{0}$. 
(ii) Now we remark that the plane $\pi$ spanned by $P$ and $L_{0}$ meets $V$ along a curve of the form $L_{0} \cup C_{0}$, with $C_{0}$ of degree 2 and $P \in C_{0}(k)$. If $C_{0}$ is a smooth conic, we have finished. Otherwise, $C_{0}$ is the union of the two lines $\left\langle P, Q_{1}\right\rangle$ and $\left\langle P, Q_{2}\right\rangle$. (Indeed it cannot contain $L_{0}$; or else, $\pi$ would be the plane $T_{0}$, which does not contain $P$, since we are in the case where $T_{0} . V=3 L_{0}$.) But only finitely many points $P \notin L_{0}$ have the property that the lines $\left\langle P, Q_{1}\right\rangle$ and $\left\langle P, Q_{2}\right\rangle$ lie on $V_{K}$. So the proof will be complete once we show that we can get infinitely many $k$-points $P \notin L_{0}$ through the above construction.

To this effect, let us consider a general plane section $Y_{1}$ of $V_{K}$ through $Q_{1}$, defined over $K$. Then $Y_{1}$ is an irreducible cubic curve with a double point at $Q_{1}$. We can assume that $P_{1}$ was chosen as the intersection of $Y_{1}$ with $\pi_{1}$ (residual to $Q_{1}$ ). Thus $P_{2}$ lies on the conjugate $Y_{2}$ of $Y_{1}$. Let $K_{P}$ be the cone over $Y_{1}$ with vertex $P$. Then it is clear that $Y_{2}$ is not contained in $K_{P}$ : otherwise, the double points of $Y_{1}$ and $Y_{2}$ would be collinear with the vertex $P$, but $P \notin L_{0}$. Therefore $Y_{2}$ meets $K_{P}$ in finitely many points. Now, clearly, $P_{2}$ is contained in the intersection $Y_{2} \cap K_{P}$. This means that, if we fix $Y_{1}$ and let $\pi_{1}$ vary, only finitely many pairs $\left(P_{1}, P_{2}\right)$ will give rise to the same point $P$. This completes the proof of the lemma.

THEOREM 1.3. Let $V \subset \mathbb{P}_{k}^{3}$ be a singular cubic surface, defined over $k$. Suppose we are in one of the following situations:

(a) $\bar{V}$ has 3 conjugate double points, and no other singularities; or

(b) $\bar{V}$ has 2 conjugate double points, and no other singularities.

Then, in Case (a), $V$ is birationally equivalent (over $k$ ) to a smooth Del Pezzo surface of degree 6. In Case (b), $V$ is birationally equivalent to a smooth Del Pezzo surface of degree 4 with a $k$-point.

Proof. As mentioned in the Introduction, we shall give two different proofs of this theorem. In this section we assume that $k \subset \mathbb{C}$ (or at least that $k$ has characteristic 0), so that we can use the classification of Proposition 0.2. (However, the result holds in general, as will be seen from the second proof, in $\S 2$.) So $\bar{V}$ has singularities of type $3 A_{1}$ or $3 A_{2}$ in Case (a), of type $2 A_{1}$ or $2 A_{2}$ in Case (b). According to Proposition 0.2 , these are the only possibilities, since the action of the Galois group $\operatorname{Gal}(\bar{k} / k)$ preserves the type of a singularity. As we shall see, the cases $3 A_{1}, 3 A_{2}$, and $2 A_{1}$ can be dealt with in a uniform way, while the case $2 A_{2}$ requires some extra work.

(a) The three double points $Q_{1}, Q_{2}, Q_{3}$ are not collinear, as we saw in the proof of Lemma 1.1. We denote by $L_{i}(i=1,2,3)$ the line joining $Q_{j}$ and $Q_{k}$ $(i \neq j, k)$. It lies on $\bar{V}$.

Now consider the minimal desingularization $f: V^{\prime} \rightarrow V$. By Proposition 0.1, we know that the canonical class of $V^{\prime}$ is represented by the total transform

$$
K_{V^{\prime}}=f^{*}\left(K_{V}\right) \text {. }
$$

Moreover, since $V$ is a cubic surface in $\mathbb{P}_{k}^{3}$, we can choose the divisor $K_{V}$ so that $-K_{V}$ is a hyperplane section which does not contain any singularity of $V$. Therefore the proper transform $L_{i}^{\prime}=f^{-1}\left[L_{i}\right]$ of each line $L_{i}$ is such that

$$
\left(f^{*}\left(-K_{V}\right) \cdot L_{i}^{\prime}\right)=\left(-K_{V} \cdot L_{i}\right)=1 .
$$

Hence $L_{i}^{\prime}$ is an exceptional divisor of the first kind. Indeed, $p_{a}\left(L_{i}^{\prime}\right)=0$ and it 
follows from (1) and (2) that

$$
\left(K_{V^{\prime}} \cdot L_{i}^{\prime}\right)=-1 .
$$

If we blow down these exceptional divisors, we get a map $g: V^{\prime} \rightarrow W$ onto a smooth surface $W$ with a canonical divisor $K_{W}$ satisfying

$$
K_{V^{\prime}}=g^{*}\left(K_{W}\right)+\sum_{i=1}^{3} L_{i}^{\prime}
$$

Then it is clear from (4), (1), and (3) that

$$
\left(K_{W}^{2}\right)=\left(g^{*}\left(K_{W}\right)^{2}\right)=\left(K_{V^{\prime}}^{2}\right)-3-2 \sum_{i=1}^{3}\left(K_{V^{\prime}} . L_{i}^{\prime}\right)=6 .
$$

We claim that $W$ is the Del Pezzo surface of degree 6 we are looking for. It suffices to show that $-K_{W}$ is ample, and this follows easily from Nakai's criterion for ampleness (see, for example, [21, Theorem 5.1, p. 30, and Remark, p. 33]). Indeed, let $D$ be any integral curve on $\bar{W}$. We have to show that $\left(-K_{W} \cdot D\right)>0$. But

$$
\left(K_{W} \cdot D\right)=\left(g^{*}\left(K_{W}\right) \cdot g^{*}(D)\right)=\left(\left(K_{V^{\prime}}-\sum L_{i}^{\prime}\right) \cdot\left(D^{\prime}+\sum v_{i} L_{i}^{\prime}\right)\right)
$$

where $D^{\prime}=g^{-1}[D]$ is the proper transform of $D$, and the $v_{i}$ are some (non-negative) integers. Then

$$
\left(-K_{W} \cdot D\right)=\left(-K_{V^{\prime}} \cdot D^{\prime}\right)+\sum\left(L_{i}^{\prime} \cdot D^{\prime}\right)+0,
$$

the closing zero being a consequence of $(3)$. Thus $\left(-K_{W} \cdot D\right) \geqslant 0$. Indeed, it follows from (1) and the choice of $-K_{V}$ that

$$
\left(-K_{V^{\prime}} \cdot D^{\prime}\right)=\left(f^{*}\left(-K_{V}\right) \cdot D^{\prime}\right)=\left(-K_{V} \cdot f\left(D^{\prime}\right)\right) \geqslant 0 .
$$

And in fact, $\left(-K_{V^{\prime}} \cdot D^{\prime}\right)>0$, unless $f\left(D^{\prime}\right)$ is reduced to a point, in which case $D^{\prime}$ is a component of the exceptional divisor $E$ above one of the singular points of $\bar{V}$, say $Q_{1}$. Thus, in order to prove that $\left(-K_{W} . D\right)>0$, it is necessary and sufficient to show that, for such a component $D^{\prime}$,

$$
\sum_{i=1}^{3}\left(L_{i}^{\prime} \cdot D^{\prime}\right)>0 .
$$

In the case $3 A_{1}$, this is clear: $E$ being irreducible, we have $D^{\prime}=E$ and $\left(L_{2}^{\prime} . E\right)=\left(L_{3}^{\prime} . E\right)=1$. In the case $3 A_{2}, D^{\prime}$ can be either of the two components of $E=E^{\prime}+E^{\prime \prime}$. Now, as follows from Lemma 3 of $[6], \bar{V}$ is equivalent to the surface with equation $x_{1} x_{2} x_{3}-x_{0}^{3}=0$, from which one can see that $\left(L_{2}^{\prime} \cdot E^{\prime}\right)=\left(L_{3}^{\prime} \cdot E^{\prime \prime}\right)=1$ (up to a permutation of $E^{\prime}$ and $E^{\prime \prime}$ ). Thus (5) holds also in this case. (At the end of the section we indicate a variant of this argument which avoids the recourse to an explicit equation.)

(b) Let $f: V^{\prime} \rightarrow V$ be the minimal desingularization of $V$. We denote by $L_{0}$ the line of $V$ that joins the two double points $Q_{1}$ and $Q_{2}$, and by $L_{0}^{\prime}$ its proper transform $f^{-1}\left[L_{0}\right]$. Then all three formulae (1), (2), and (3), are valid. So $L_{0}^{\prime}$ is an exceptional divisor of the first kind. If we blow it down, we get a map $g: V^{\prime} \rightarrow W$, 
and the analogue of (4) is

$$
K_{V^{\prime}}=g^{*}\left(K_{W}\right)+L_{0}^{\prime}
$$

It follows that $\left(K_{W}^{2}\right)=\left(K_{V^{\prime}}^{2}\right)-1-2\left(K_{V^{\prime}} \cdot L_{0}^{\prime}\right)=4$. Now, the same argument as was used under (a) shows that $-K_{W}$ is ample if and only if

$$
\left(L_{0}^{\prime} . D^{\prime}\right)>0
$$

for any component $D^{\prime}$ of the exceptional divisor $E$ above either $Q_{1}$ or $Q_{2}$.

So, in the case $2 A_{1}, W$ is a Del Pezzo surface, since $D^{\prime}=E$ is irreducible and $\left(L_{0}^{\prime} . E\right)>0$. But this is not true in the case $2 A_{2}$ ! Indeed, it follows from Lemma 3 of [6] that, in this case, $\bar{V}$ is equivalent to a surface with equation

$$
x_{1} x_{2} x_{3}-\varphi\left(x_{0}, x_{1}\right)=0,
$$

from which one can see that $E=E^{\prime}+E^{\prime \prime}$ with $\left(L_{0}^{\prime} \cdot E^{\prime}\right)=1$, but $\left(L_{0}^{\prime} \cdot E^{\prime \prime}\right)=0$. Thus $\left(5^{\prime}\right)$ does not hold for $D^{\prime}=E^{\prime \prime}$, and $-K_{W}$ is not ample.

But, by Lemma 1.2 , we can choose a point $P \in V(k)$ such that the plane $\left\langle P, Q_{1}, Q_{2}\right\rangle$ meets $V$ in the union $L_{0} \cup C_{0}$, where $C_{0}$ is a smooth conic. Let $\pi: V^{\prime \prime} \rightarrow V^{\prime}$ be the blowing up of $V^{\prime}$ at the point $f^{-1}(P)$. Then an easy verification shows that the proper transform $C_{0}^{\prime \prime}=(f \circ \pi)^{-1}\left[C_{0}\right]$ is an exceptional divisor of the first kind, as well as $L_{0}^{\prime \prime}=(f \circ \pi)^{-1}\left[L_{0}\right]=\pi^{-1}\left[L_{0}^{\prime}\right]$. Moreover, $C_{0}^{\prime \prime} \cap L_{0}^{\prime \prime}=\varnothing$, since $C_{0}$ and $L_{0}$ are not tangent. (Remember $f$ is simply a blowing up.) We can therefore blow down both $C_{0}^{\prime \prime}$ and $L_{0}^{\prime \prime}$, thus obtaining a morphism $h: V^{\prime \prime} \rightarrow Z$ onto a smooth surface $Z$, which is the required Del Pezzo surface of degree 4:

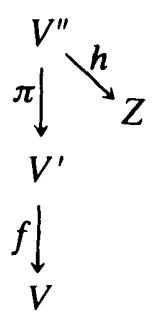

Indeed, the anticanonical divisor $-K_{Z}$ verifies Nakai's criterion for ampleness, as can be seen from the fact that $f^{-1}\left[C_{0}\right]$ meets the exceptional component $E^{\prime \prime}$ above $Q_{1}$, and from the relation

$$
K_{V^{\prime \prime}}=h^{*}\left(K_{Z}\right)+C_{0}^{\prime \prime}+L_{0}^{\prime \prime}
$$

For let $D$ be any integral curve on $\bar{Z}$. We have to show that $\left(-K_{Z} \cdot D\right)>0$. But

$$
\left(K_{Z} \cdot D\right)=\left(h^{*}\left(K_{Z}\right) \cdot h^{*}(D)\right)=\left(K_{V^{\prime \prime}}-C_{0}^{\prime \prime}-L_{0}^{\prime \prime}\right) \cdot\left(D^{\prime \prime}+\mu C_{0}^{\prime \prime}+v L_{0}^{\prime \prime}\right),
$$

where $D^{\prime \prime}=h^{-1}[D]$. Hence, as before,

$$
\left(-K_{Z} \cdot D\right)=\left(-K_{V^{\prime \prime}} \cdot D^{\prime \prime}\right)+\left(C_{0}^{\prime \prime} \cdot D^{\prime \prime}\right)+\left(L_{0}^{\prime \prime} \cdot D^{\prime \prime}\right)
$$

It suffices to show that $\left(-K_{V^{\prime}} \cdot D^{\prime \prime}\right)>0$. We may assume that $D^{\prime \prime} \neq E_{0}=$ $(f \circ \pi)^{-1}(P)$, since $\left(K_{V^{\prime}} \cdot E_{0}\right)=-1$. Let $D^{\prime}=\pi\left(D^{\prime \prime}\right)$, so that

$$
\pi^{*}\left(D^{\prime}\right)=D^{\prime \prime}+\lambda E_{0}
$$


where $\lambda$ is the multiplicity of $D^{\prime}$ at $f^{-1}(P)$. Thus

$$
\begin{aligned}
\left(-K_{V^{\prime \prime}} \cdot D^{\prime \prime}\right) & =\left(-K_{V^{\prime \prime} \cdot}\left(\pi^{*}\left(D^{\prime}\right)-\lambda E_{0}\right)\right) \\
& =\left(\left(-\pi^{*}\left(K_{V^{\prime}}\right)-E_{0}\right) \cdot \pi^{*}\left(D^{\prime}\right)\right)+\lambda\left(K_{V^{\prime \prime}} \cdot E_{0}\right) \\
& =\left(-K_{V^{\prime}} \cdot D^{\prime}\right)-\left(E_{0} \cdot \pi^{*}\left(D^{\prime}\right)\right)-\lambda \\
& =\operatorname{deg} f\left(D^{\prime}\right)-0-\lambda \\
& =\operatorname{deg} f\left(D^{\prime}\right)-\operatorname{mult}_{P} f\left(D^{\prime}\right)>0,
\end{aligned}
$$

since the multiplicity of an irreducible curve at a point is always less than its degree.

$A$ variant. This proof can be described in a more conceptual way, provided we refer to Part II for some notation. In essence we shall use the same ingredients, but the argument looks very much simpler than the above. This is because we do not prove the assertions we make about the diagrams of irreducible roots and lines. Justifying these assertions as is done in $\S 5$ for degree 4 , i.e. without using [6], would require a certain amount of work!

Consider the minimal desingularization $f: V^{\prime} \rightarrow V$. We know from Example 0.5 that $V^{\prime}$ is a generalized Del Pezzo surface of degree 3. Its exceptional curves of the first kind are the proper transforms of the lines of $\bar{V}$. Its irreducible roots correspond to the vertices of the Dynkin diagram $3 A_{1}, 3 A_{2}, 2 A_{1}$, or $2 A_{2}$, as the case may be.

(a) Blow down the three lines $L_{i}^{\prime}$. This yields a surface $W$, which is still a generalized Del Pezzo surface, with degree 6 (cf. § 7). Moreover $W$ does not contain any irreducible root. By Criterion 7.3 this is clear in the case $3 A_{1}$ and, in the case $3 A_{2}$, it amounts to proving (5). But it can be shown that the diagram of irreducible roots and lines looks as follows (the notation is explained in §6):

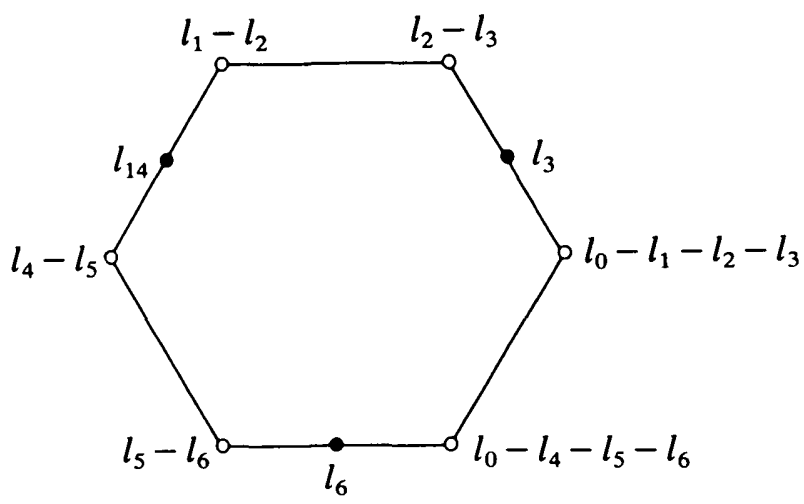

Blowing down the three lines, we obtain a diagram of the form

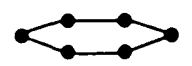

with no roots. By Proposition 0.6, this corresponds to a smooth Del Pezzo surface of degree 6.

(b) Blow down the line $L_{0}^{\prime}$. This yields a generalized Del Pezzo surface $W$ with degree 4 . In the case $2 A_{1}, W$ does not contain any irreducible root and we 
conclude as above. In the case $2 A_{2}$, it can be shown that the diagram of irreducible roots and lines (cf. $\S 6$; by definition, $q_{i}=2 l_{0}-\sum_{h \neq i} l_{h}$ ) looks as follows:

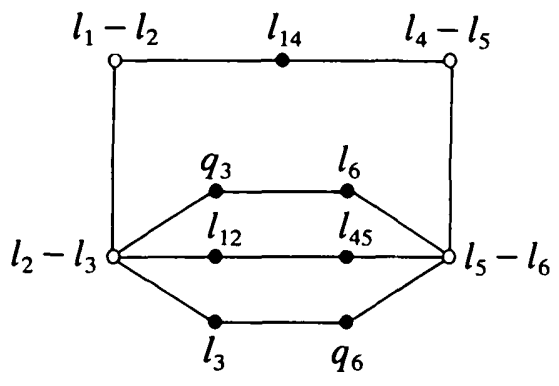

Now $W$ has been obtained by blowing down $l_{14}$. Therefore, by Criterion 7.3 , it is of the following type:

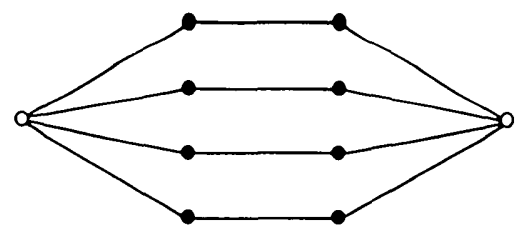

In other words, $W$ belongs to Type 3 of Proposition 6.1. (It is an Iskovskih surface.) As in the above proof, we can blow up a suitable $k$-point of $W$, not lying on any irreducible root. It follows from Proposition 0.4 that the surface we obtain in this way is a generalized Del Pezzo surface of degree 3 and of type $2 A_{1}$. Now this situation has already been considered. (In Example 0.7(a) we saw that this surface is the minimal desingularization of a cubic surface of type $2 A_{1}$.)

Note. The diagram for the case $2 A_{1}$ is almost impossible to draw! As a consolation prize we can offer the diagram for the case $3 A_{1}$ (see facing page). (For lack of space, the following shorter symbols have been used: $a=l_{13}, b=l_{15}$, $c=l_{35}$.)

\section{Explicit resolution by means of a Cremona transformation}

The proof of Theorem 1.3 given in $\S 1$ has the advantage that it is very natural once we know a good local description of the singularities. But this description, provided by Proposition 0.2, is not available in positive characteristic. On the other hand, Part (a) of the theorem was already proved in [17], by means of an explicit Cremona transformation. This section is devoted to a proof of Part (b) of the theorem which is in a similar vein, and is even simpler than that of Part (a) in [17]. This is due essentially to the fact that the transformation we use here is an involution. Of course this tremendously simplifies the description of the inverse Cremona transformation! We begin by describing this rational map $\Phi: \mathbb{P}^{3} \ldots \rightarrow \mathbb{P}^{3}$, which turns out to be one of the most classical Cremona transformations of $\mathbb{P}^{3}$.

Fix a plane $\pi \subset \mathbb{P}^{3}$, and let $C \subset \pi$ be a conic (not necessarily irreducible). 


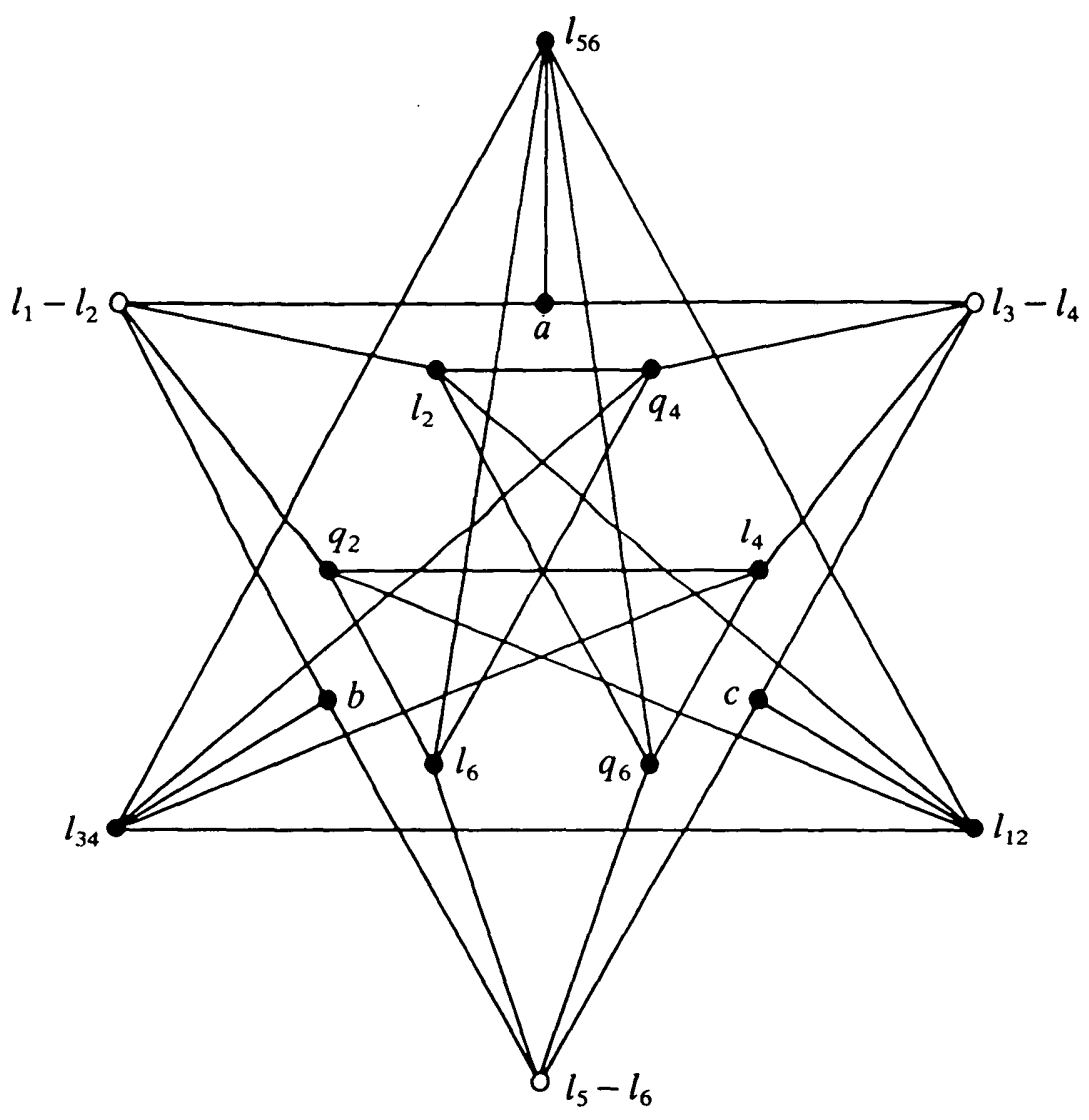

Choose a point $P \notin \pi$, and consider the linear system $M$ of all quadrics containing $C \cup\{P\}$. This defines a rational map $\Phi: \mathbb{P}^{3} \ldots \rightarrow \mathbb{P}^{3}$, which is easy to describe explicitly: if we denote by $(x, y, z, t)$ the coordinates in $\mathbb{P}^{3}$, we can assume that $P=(0,0,0,1)$ and that $\pi$ is given by the equation $t=0$. Then $\mathcal{M}$ is generated by the following divisors: $\{x t, y t, z t, \varphi(x, y, z)\}$, where $\varphi(x, y, z)=0$ is the equation of $C$ in $\pi$. Thus we can write

$$
\Phi(x, y, z, t)=(x t, y t, z t, \varphi(x, y, z)),
$$

and a trivial verification shows that $\Phi^{-1}$ is given by the same formula, that is, $\Phi^{-1}=\Phi$.

Remark. Note that $C$ may even be non-reduced. In fact, the classical quadratic transformation ( $\sigma$-process) is the situation in which $C$ is a double line: written projectively, the usual coordinate change $X=x / z, Y=y / z, Z=z$ becomes

$$
(x, y, z, t) \mapsto\left(x t, y t, z^{2}, z t\right) ;
$$

in this case, $C$ is the double line $z^{2}=0$ in the plane $t=0$. Written like this, the transformation is not an involution; so it is an advantage to interchange the last two coordinates $Z$ and $T$.

The effect of $\Phi$ on $\mathbb{P}^{3}$ is easily obtained from (1): 
Lemma 2.1. Let $K$ be the cone over $C$ with vertex $P$.

(a) $\Phi$ is undefined only on $C \cup\{P\}$.

(b) $\Phi$ maps $\pi \backslash C$ into $P$.

(c) $\Phi$ blows down each (open) generating line of the cone $K$, say

$$
\left\{\left(\lambda x_{0}, \lambda y_{0}, \lambda z_{0}, 1\right) \mid \lambda \neq 0, \varphi\left(x_{0}, y_{0}, z_{0}\right)=0\right\} \text {, }
$$

into the corresponding point $\left(x_{0}, y_{0}, z_{0}, 0\right) \in C$.

(d) Conversely, $\Phi$ blows up $P$ into $\pi$, and each point $Q_{0}=\left(x_{0}, y_{0}, z_{0}, 0\right) \in C$ into the corresponding generating line $\left\langle P, Q_{0}\right\rangle$ of $K$.

(e) Let $\Sigma=\mathbb{P}^{3} \backslash(\pi \cup K)$. Then $\left.\Phi\right|_{\Sigma}$ is an isomorphism of $\Sigma$ onto itself.

(f) $A$ general line $L$ of $\mathbb{P}^{3}$ is in correspondence with an intersection of two quadrics in $M$, that is, with the union of $C$ (the fuxed part) and a variable conic $\Phi^{-1}[L]$ passing through $P$ and meeting $C$ in two points. (Only one if $C$ is a double line, but then $\Phi^{-1}[L]$ is tangent to $\pi$.)

Proof. All these assertions are easy to verify. We prove only (e), since it is crucial for what follows. In view of (a), we know that $\Phi$ is certainly defined everywhere off $\pi \cup K$. Moreover $\Phi(\Sigma) \subset \Sigma$. Indeed, since $\Phi(x, y, z, t) \in \pi$, we have $\varphi(x, y, z)=0$; whence $(x, y, z, t) \in K$. And if $\Phi(x, y, z, t) \in K \backslash \pi$, then $\varphi(x t, y t, z t)=0$ and $\varphi(x, y, z) \neq 0$; hence $t=0$; therefore $(x, y, z, t) \in \pi$. Now, since $\Phi^{2}=\mathrm{id}$, it follows that $\left.\Phi\right|_{\Sigma}: \Sigma \rightarrow \Sigma$ is an isomorphism.

We can now restate Theorem $1.3(\mathrm{~b})$, in a more precise formulation:

THEOREM 2.2. Let $V \subset \mathbb{P}_{k}^{3}$ be a cubic surface having two conjugate singular points $Q_{1}, Q_{2}$, and no other singularities. Let $\pi$ be a $k$-rational plane through $L_{0}=\left\langle Q_{1}, Q_{2}\right\rangle$, and write $\pi . V=L_{0}+C$, where $C$ is a conic. $C$ may be reducible, but we assume that $\pi$ has been chosen so that $C \neq L_{0}$. Let $P \in V(k)$ be such that $P \notin \pi$ and $P$ does not lie on any line of $\vec{V}$. (The existence of such a point is guaranteed by Lemma 1.2!) Then the following assertions hold.

(a) The image $W=\Phi[V]$ of $V$ under the quadratic transformation $\Phi$ defined by $C$ and $P$ is a cubic surface. If $V$ is defined by equation (2) below, then $W$ is defined by (3).

(b) If the $Q_{i}$ are of type $A_{1}$, that is, if the tangent cone at each point $Q_{i}$ is non-degenerate, then $W$ is smooth. Otherwise, $\bar{W}$ contains exactly two conjugate double points, which are of type $A_{1}$.

(c) If the $Q_{i}$ are of type $A_{1}$, then $W$ contains three $k$-rational lines in a plane ( $\pi^{\prime}$, say). Moreover, $\bar{W}$ contains the conjugate lines $L_{1}=\left\langle P, Q_{1}\right\rangle$ and $L_{2}=$ $\left\langle P, Q_{2}\right\rangle$, so that $W$ has at least two $k$-rational tritangent planes, viz. $\pi^{\prime}$ and $\pi_{P}=\left\langle P, Q_{1}, Q_{2}\right\rangle$.

REMARK. The classification of singularities does not play any role in the proof of this theorem. However, in order to allow comparison with Theorem 1.3(b), we refer to 'type $A_{1}$ ' for the case in which the tangent cone is non-degenerate.

Proof. (a) The degree of $W$ is the intersection number of $W$ with a generic line of $\mathbb{P}^{3}$. By Lemma 2.1(f), this is also the number of variable intersections of $V$ with a conic passing through $P$ and meeting $C$ in two points. Now, it is easy to see that there are three movable intersection points off the base locus of $\Phi$. Hence $\operatorname{deg} W=3$. 
This can also be seen explicitly. Indeed we can make the following assumptions:

$P=(0,0,0,1)$

$\pi$ is the plane with equation $t=0$;

$L_{0}$ is the line with equations $x=t=0$.

Then the equation of $V$ is as follows:

$$
t^{2} \alpha(x, y, z)+t \beta(x, y, z)+x \varphi(x, y, z)=0 .
$$

The Cremona transformation $\Phi: \mathbb{P}^{3} \ldots \mathbb{P}^{3}$ can be viewed either as a birational map from $\mathbb{P}^{3}$ into itself, or as a birational map from a first space $\mathbb{P}=\mathbb{P}^{3}$ into another space $\mathbb{P}^{\prime} \simeq \mathbb{P}^{3}$. The former point of view is useful in particular when we wish to emphasize the fact that $\Phi$ is an involution. However, when writing down explicit equations, we shall adopt the latter point of view, using coordinates $(x, y, z, t)$ for $\mathbb{P}$, and $(X, Y, Z, T)$ for $\mathbb{P}^{\prime}$. This will prevent some confusion. (The two ways of thinking can be reconciled by thinking of $\mathbb{P}$ and $\mathbb{P}^{\prime}$ as being superposed like two leaves of a book. Thus, for instance, the plane $\pi$ is defined indifferently by the equation $t=0$ or by $T=0$.) In particular, the equation of $V$ will be in the coordinates of $\mathbb{P}$, while the equation of $W$ will be in those of $\mathbb{P}^{\prime}$. This equation is easily obtained from (2), since we know $\Phi^{-1}$. It suffices to substitute

$$
(x, y, z, t)=(X T, Y T, Z T, \varphi(X, Y, Z))
$$

in (2), and we get

$$
\varphi(X, Y, Z) \alpha(X, Y, Z)+T \beta(X, Y, Z)+T^{2} X=0
$$

(b) In studying the singularities of $W$, we can forget about the ground field and work over the algebraic closure of $k$, remembering only that $Q_{1}$ and $Q_{2}$ must have tangent cones of the same type, both degenerate or both non-degenerate. Thus we are free to suppose that

$$
Q_{1}=(0,0,1,0) \text { and } Q_{2}=(0,1,0,0) .
$$

(This assumption serves to simplify the end of the proof.) Now we observe that

$$
\varphi=a x^{2}+b x y+c x z+d y z
$$

with $d \neq 0$, since $C$ contains $Q_{1}$ and $Q_{2}$, but does not contain $L_{0}$. Up to a linear change of coordinates of the form $y=y^{\prime}-\lambda x, z=z^{\prime}-\mu x$ (which induces the identity on $L_{0}$ and leaves $P$ fixed), we may assume that $\varphi=\varepsilon x^{2}+y z$. The conic $C$ is smooth if and only if $\varepsilon \neq 0$; in this case we may assume, up to a transformation of the form $y=\varepsilon y^{\prime}$ (which leaves the $Q_{i}$ fixed), that $\varepsilon=1$. Therefore we may assume that

$$
\varphi(x, y, z)=\varepsilon x^{2}+y z, \quad \text { with } \varepsilon=0 \text { or } 1 .
$$

Furthermore, the coefficients of $y^{2}$ and $z^{2}$ in $\beta(x, y, z)$ are equal to zero, since $Q_{1}$ and $Q_{2}$ are double. Thus we can write

$$
\begin{aligned}
& \alpha(x, y, z)=a x+b y+c z, \\
& \beta(x, y, z)=d x^{2}+e x y+f x z+g y z .
\end{aligned}
$$

It is interesting to remark that $\alpha(x, y, z)=0$ is the equation of the tangent plane at $P$. Finally, we note that the following inequalities hold:

$$
b \neq 0, \quad c \neq 0, \quad g \neq 0
$$


Indeed, suppose $c=0$; then the line $x=y=0$ is entirely contained in $V$, contrary to the assumption that $P$ does not belong to any line of $V$. Similarly, the last inequality holds because the line $x=b y+c z=0$ does not lie on $V$.

With these preliminaries, it is an easy matter to study the singularities of $W$. Indeed, it follows from Lemma 2.1(e) that $W$ is smooth everywhere, except possibly on $\pi \cup K \subset \mathbb{P}^{\prime}$, where $\pi$ is defined by the equation $T=0$, and $K$ by $\varphi(X, Y, Z)=0$. From (3) we see that a singular point of $W$ is a solution of the following system:

$$
\left\{\begin{array}{l}
\varphi_{X}^{\prime} \alpha+\varphi \alpha_{X}^{\prime}+T \beta_{X}^{\prime}+T^{2}=0 \\
\varphi_{Y}^{\prime} \alpha+\varphi \alpha_{Y}^{\prime}+T \beta_{Y}^{\prime}=0 \\
\varphi_{Z}^{\prime} \alpha+\varphi \alpha_{Z}^{\prime}+T \beta_{Z}^{\prime}=0 \\
\beta+2 T X=0 .
\end{array}\right.
$$

If $T=0$, we find that $\beta=0$ and $\operatorname{Jac}(\varphi \alpha)=0$. Moreover, (3) implies that $\varphi \alpha=0$. Therefore (even in characteristic 3 ) a singular point of $W$ corresponds to a singularity of the curve with equation $\varphi \alpha=0$. Now we distinguish two cases.

(i) Suppose the system $\varphi=\alpha=\beta=0$ has a non-trivial solution $\left(X_{0}, Y_{0}, Z_{0}\right)$. Then it follows from (2) that $V$ contains a line passing through $P$, namely $\left\{\left(\lambda X_{0}, \lambda Y_{0}, \lambda Z_{0}, 1\right) \mid \lambda \in \bar{k}\right\}$, and this contradicts one of our assumptions.

(ii) A singularity of the curve with equation $\varphi \alpha=0$ must therefore correspond to a singularity of $C$, which is possible only if $\varepsilon=0$ in (4). Then $\left(X_{0}, Y_{0}, Z_{0}\right)=$ $(1,0,0)$; so, by (6), $\beta$ can vanish at this point only if $d=0$. But, in this case, it follows from (2) that $(1,0,0,0)$ is a singular point of $V$, contrary to the assumption that there are no singularities other than the $Q_{i}$.

Now suppose $T \neq 0$, but $\varphi(X, Y, Z)=0$. Then it follows from (3) that $\beta+T X=0$. By (8), a singularity can occur only if $\beta+2 T X=0$. Hence $X=0$. Therefore $\beta=0$ and, using (6) and (7), we see that $Y Z=0$. Note that, as a consequence of (3), (4), and (6), $W$ contains the two lines $L_{1}=\{X=Y=0\}$ and $L_{2}=\{X=Z=0\}$, which are the blow-ups of $Q_{1}$, respectively $Q_{2}$ (cf. Lemma 2.1(d)). We have to look for singularities of $W$ on one of these lines, say $L_{1}$ (by symmetry, this is enough). The last two derivatives in (8) give no information. The first two imply that

$$
\left\{\begin{array}{l}
f Z+T=0 \\
c Z+g T=0
\end{array}\right.
$$

This is possible only if

$$
f g=c .
$$

And this is precisely the condition for the tangent cone at $Q_{1}$ to be degenerate (its equation is $c t^{2}+f t x+g t y+x y=0$ ). This proves that $W$ is smooth in the case $2 A_{1}$. In the remaining case, the tangent cone at $Q_{2}$ must also be degenerate, that is,

$$
e g=b \text {, }
$$

since $Q_{1}$ and $Q_{2}$ were conjugate. Then $W$ is indeed singular, with the two singular points

$$
Q_{1}^{\prime}=(0,0,1,-f) \text { and } Q_{2}^{\prime}=(0,1,0,-e) .
$$

In order to complete the proof, it suffices to check that the tangent cone at $Q_{1}^{\prime}$ 
is non-degenerate. To this effect, we change coordinates, as follows: $T=U-f Z$, and compute the initial form after setting $Z=1$. We obtain

$$
(\varepsilon c-d f) X^{2}+(a-e f) X Y+b Y^{2}-f X U+g Y U .
$$

Substituting $f g$ for $c$, and $e g$ for $b$, we find that this quadratic form is degenerate only if

$$
d g=a+g^{2} \varepsilon
$$

But this is impossible, since the conditions (10), (11) and (12) imply that $V$ was reducible; a contradiction. Indeed, the equation (2) can then be written in the form

$$
(g t+x)\{t \alpha(x, y, z)+g \varphi(x, y, z)\}=0 .
$$

(c) Finally we have to prove that, in the case $2 A_{1}, W$ contains three $k$-rational lines in a plane. This can be seen as follows.

As $P$ is an isolated base point for the linear system $\mu$, it must be blown up into a $k$-rational line $L_{P}$ on $W$. Since $\Phi$ blows up $P$ into $\pi$ (Lemma 2.1(d)), $L_{P}$ lies in this plane. One sees easily that $L_{P}$ is defined by the equations $T=\alpha(X, Y, Z)=0$.

We noticed in the course of the proof that $L_{1}=\left\langle P, Q_{1}\right\rangle$ and $L_{2}=\left\langle P, Q_{2}\right\rangle$ lie on $\bar{W}$. Thus the plane $\pi_{P}=\left\langle P, Q_{1}, Q_{2}\right\rangle$, with equation $X=0$, contains a third line, which is $k$-rational. Call it $L^{\prime}$. According to (3), (5), and (6), it is defined by the equations $X=b Y+c Z+g T=0$. Thus $L^{\prime} \neq L_{P}$; in fact, $L^{\prime}$ is the transform of the conic residual to $L_{0}$ in the plane $\pi_{P}$.

The lines $L_{P}$ and $L^{\prime}$ meet in the point $(0, c,-b, 0)$. Hence they determine a plane $\pi^{\prime}$, with equation $a X+b Y+c Z+g T=0$, which contains a third $k$-rational line $L^{\prime \prime}$ of $W$. This completes the proof of the theorem.

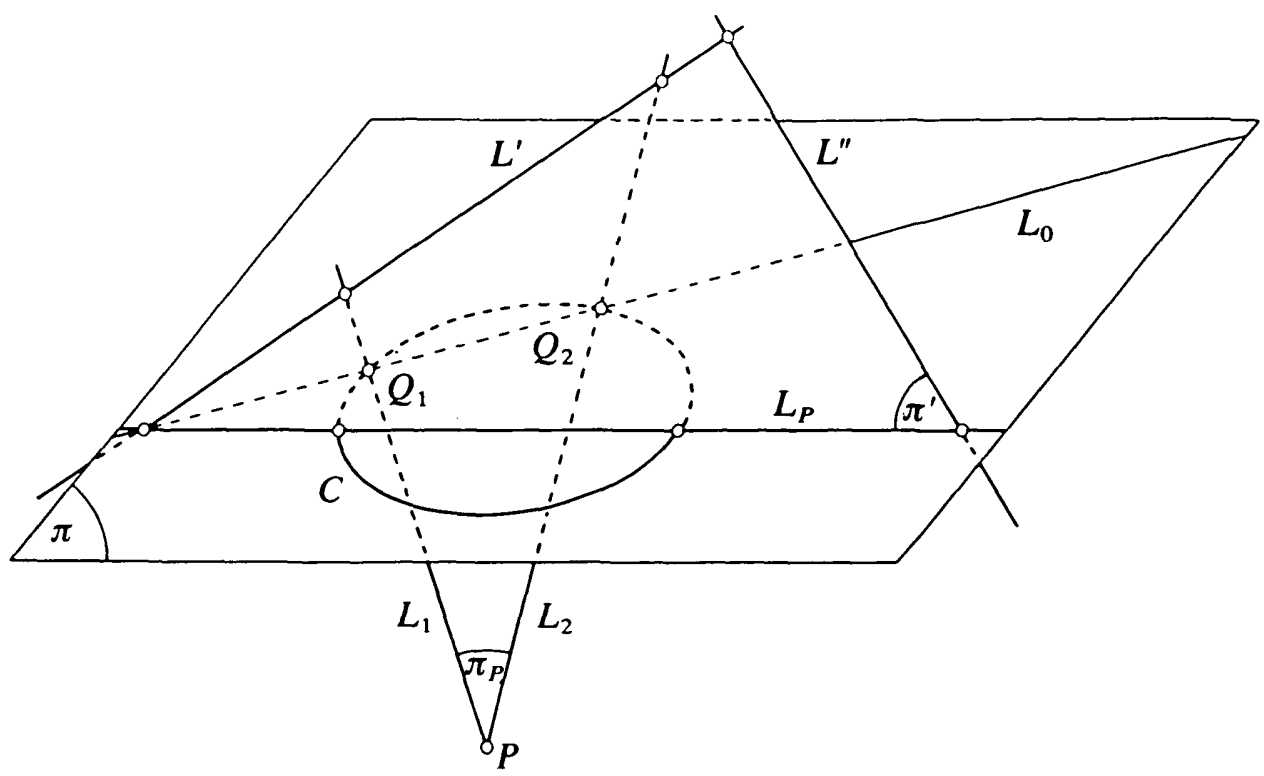

FIG. 1 
Remarks. (a) The existence of three $k$-rational lines on $W$ is in agreement with a theorem of Iskovskih [25], to the effect that a conic bundle surface with invariant 4 is a Del Pezzo surface of degree 4 only if it can be fibred in two different ways. After blowing down one of the lines in $\pi^{\prime}$, there remain two different fibrations (around either of the other two lines).

(b) Of course, the transformation $\Phi$ can also be used if $V$ contains some further singular points. By Lemma 2.1(e), these are simply preserved.

(c) It is clear that Theorem 1.3(b) is a consequence of Theorem 2.2, since we can apply the transformation $\Phi$ twice if necessary and blow down one of the $k$-rational lines, so as to obtain a smooth intersection of two quadrics in $\mathbb{P}_{k}^{4}$.

(d) As a matter of fact, this too can be done quite explicitly. Indeed, the line $L_{P}$ lies on $W$ because $P$ is a base point of the linear system $M$. Thus we can blow down $L_{P}$ simply by forgetting $P$ in the description of $\Phi$, which amounts to considering a bigger linear system, say $\mathcal{N}$, generated by $\left\{x t, y t, z t, \varphi(x, y, z), t^{2}\right\}$. This is the content of the next corollary:

CoRollaRy 2.3. Let $V \subset \mathbb{P}_{k}^{3}$ be a cubic surface having two conjugate singular points of type $A_{1}$, and no other singularities.

(a) $V$ is birationally equivalent to a smooth intersection of two quadrics $U$ in $\mathbb{P}_{k}^{4}$. If $t=\varphi(x, y, z)=0$ is the equation of the conic $C$, which is chosen as in Theorem 2.2 , the birational equivalence $\Psi: V \rightarrow U$ is given by

$$
\Psi:(x, y, z, t) \mapsto\left(x t, y t, z t, \varphi(x, y, z), t^{2}\right) .
$$

(b) $\bar{U}$ contains two conjugate lines, $\tilde{L}_{1}$ and $\tilde{L}_{2}$, which meet in a point $\widetilde{P} \in U(k)$.

(c) If $V$ is defined by (2), then $U$ is defined in $\mathbb{P}_{k}^{4}$, with coordinates $(\xi, \eta, \zeta, \tau, v)$, by the following system of equations:

$$
\left\{\begin{array}{l}
\varphi(\xi, \eta, \zeta)-\tau v=0 \\
\alpha(\xi, \eta, \zeta) v+\beta(\xi, \eta, \zeta)+\xi \tau=0
\end{array}\right.
$$

Proof. (a) For the purpose of the proof, we choose a $k$-point $P \in V(k)$ satisfying the conditions imposed in Theorem 2.2. (This point plays no role in the statement, for it is clear that the linear system $\mathcal{N}$, which defines $\Psi$, consists of all quadrics that contain the conic $C$.) Of course we can assume that $P=(0,0,0,1)$, and that $V$ is given by equation (2). Further, since $\Psi$ is obviously defined over $k$, we can forget about the ground field and work over $\bar{k}$. Thus we shall adopt the same notation as in the proof of Theorem 2.2.

Let $U$ be the intersection of two quadrics determined by (13). It is not hard to check that $\Psi[V] \subset U$. We shall prove that $U$ is obtained from $W=\Phi[V]$ by blowing down the line $L_{P}$. Since, by Theorem $2.2, W$ is smooth, this will imply that $U$ is smooth and irreducible, whence the result follows.

Now, we can define a morphism $s: W \rightarrow U$ as follows: the image of $(X, Y, Z, T) \in W$ is

$$
(\xi, \eta, \zeta, \tau, v)= \begin{cases}\left(X T, Y T, Z T, T^{2}, \varphi(X, Y, Z)\right) & \text { on } W_{1} \\ (X \alpha, Y \alpha, Z \alpha, T \alpha,-T X-\beta) & \text { on } W_{2}\end{cases}
$$

where

$$
W_{1}=W \backslash\{T=\varphi(X, Y, Z)=0\},
$$


and

$$
W_{2}=W \backslash\{\alpha(X, Y, Z)=T X+\beta(X, Y, Z)=0\} .
$$

This map is well defined. Indeed, since $W$ is smooth, it follows from (3) that $W_{1} \cup W_{2}=W$. Moreover, on $W_{1} \cap W_{2}$, it follows from (3) that $T=0$ if and only if $\alpha(X, Y, Z)=0$. Hence it is immediate that the two descriptions of $s$ coincide on $W_{1} \cap W_{2}$. Furthermore, the image of $s$ is in $U$.

An easy verification shows that $\Psi=s \circ \Phi$. Let $p: U \ldots W$ be the projection $(X, Y, Z, T)=(\xi, \eta, \zeta, \tau)$, which is defined everywhere, except at the point $P^{\prime}=(0,0,0,0,1)$ :

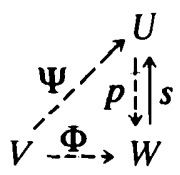

Let $W^{\prime}=W \backslash\{T=\alpha(X, Y, Z)=0\}$ and $U^{\prime}=U \backslash\left\{P^{\prime}\right\}$. Then $s$ maps $W^{\prime}$ into $U^{\prime}, p$ maps $U^{\prime}$ into $W^{\prime}$. Moreover, $s^{\prime}=\left.s\right|_{w^{\prime}}$ and $p^{\prime}=\left.p\right|_{U^{\prime}}$ satisfy the relations

$$
s^{\prime} \circ p^{\prime}=\mathrm{id}_{U^{\prime}} \text { and } p^{\prime} \circ s^{\prime}=\mathrm{id}_{W^{\prime}} \text {. }
$$

Finally, the line $L_{P}$ with equations $T=\alpha(X, Y, Z)=0$ is mapped into the point $P^{\prime}$, which is smooth on $U$. This can be verified directly on the system (13): one needs to know only that $\alpha$ is not identically zero, but this is clear since $\alpha(x, y, z)=0$ is the equation of the tangent plane to $V$ at $P$.

This shows that $s$ is the map that blows down $L_{P}$ on $W$. Since $U$ is the image, and $W$ is smooth, we see that $U$ is smooth and irreducible, and $\Psi=s \circ \Phi$ has the required properties.

(b) By Theorem 2.2(c), $\bar{W}$ contains two conjugate lines, $L_{1}$ and $L_{2}$, through $P \in W(k)$. Since the morphism $s: W \rightarrow U$, which blows down $L_{P}$, is defined over $k$, we see that $\tilde{P}=s(P) \in U(k)$, and $\bar{U}$ contains the two conjugate curves $\tilde{L}_{1}=s\left(L_{1}\right)$ and $\tilde{L}_{2}=s\left(L_{2}\right)$ through $\tilde{P}$. It remains to show that $\tilde{L}_{1}$ and $\tilde{L}_{2}$ are straight lines, and for this we can again work over $\bar{k}$. Then $\tilde{P}=(0,0,0,1,0)$, and $L_{1}=\{X=Y=0\}$ is mapped into $\tilde{L}_{1}=\{\xi=\eta=v=0\}$, whence the result follows.

(c) If $V$ is given by equation (2), then the argument of Part (a) shows also that $\Psi[V]$ is defined by the system (13). At first sight the result seems to depend on the fact that $P=(0,0,0,1) \in V(k)$ satisfies certain particular conditions. But since the linear system $\mathcal{N}$ is independent of the choice of $P$, we have certainly shown that $\Psi[V]$ is a smooth intersection of two quadrics. Now, trivially, it is contained in the variety $U$ defined by (13). Hence $\Psi[V]=U$.

Remarks. (a) As we saw at the beginning of the proof, and again in Part (c), the statement of Corollary 2.3 makes no reference to the $k$-point $P$ of Theorem 2.2. If no suitable point is explicitly known (remember that it must not lie on any line of $\bar{V}$ ), we may be unable to write the equation of $V$ in the form (2). But it is not difficult to compute the expression that replaces the second equation in (13). Even if $V$ is defined by an equation of the form (2), the inequalities (7) may fail to hold. But in all cases $\Psi[V]$ is smooth! This remark will be used in Example 3.3 below. 
(b) It is not difficult to convince oneself from Lemma 2.1 that the effect of the transformation $\Psi$ on $W$ is as described in the proof of Theorem 1.3(b). In the case $2 A_{1}, \Phi$ blows up $P$ into $L_{P}$, and the $Q_{i}$ into the corresponding irreducible roots. Then it blows down the proper transform of $L_{0}$ into $P$, and carries the irreducible roots into $L_{1}$ and $L_{2}$. Now, the morphism $s$ blows down $L_{P}$ into $P^{\prime}$ and carries $L_{1}, L_{2}$, and $P$ into $\tilde{L}_{1}, \tilde{L}_{2}$, and $\tilde{P}$ respectively. Therefore $\Psi$ blows up the $Q_{i}$, and then blows down the proper transform of $L_{0}$ into $\tilde{P}$. In addition, it carries $P$ into $P^{\prime}$.

\section{Miscellaneous applications}

Theorem 1.3 has a number of applications to arithmetical questions. We may recall the following result, which was also discussed in [17, pp. 55-56]:

Proposition 3.1 (Skolem). The class of singular cubic surfaces defined over a number field $k$ satisfies the Hasse principle.

Proof. This can be viewed as a consequence of Theorem 1.3(a). Indeed, by Lemma 1.1 and an elementary discussion, we are reduced to the case of three conjugate double points, and hence to a smooth Del Pezzo surface of degree 6 . Now, for such a surface, the Hasse principle is well known to hold, for instance because it is the compactification of a principal homogeneous space over a two-dimensional $k$-torus [32, Chapter IV, $\S 8.6$ and 8.7].

Proposition 3.2. Let $V \subset \mathbb{P}_{k}^{3}$ be a singular, absolutely irreducible, cubic surface. Suppose $V$ is neither a cone, nor a surface with precisely two conjugate double points. Let $Z$ be any smooth projective model of $V$. Then the following assertions hold:

(a) if $Z(k) \neq \varnothing$ then $Z$ is $k$-rational;

(b) the $\mathrm{Gal}(\bar{k} / k)$-module $\mathrm{Pic} \bar{Z}$ is stably a permutation module;

(c) the Néron-Severi torus of $Z$ (dual to Pic $\bar{Z}$ ) is stably rational;

(d) if $k$ is a local or global field of characteristic 0 , then the Chow group $A_{0}(Z)=\operatorname{ker}\left(C_{0}(Z) \rightarrow \mathbb{Z}\right)$ is trivial.

Proof. (a) By Nishimura's lemma (Lemma 1.0), $V(k) \neq \varnothing$. Hence the assertion follows from Lemma 1.1. (If the singularities of $V$ are not isolated then $V$ has a double line, and it suffices to project from any $k$-point on it.)

(b) This is a consequence of Property (a) for the class of surfaces we consider, at least if $\operatorname{char}(k)=0$ (see [15, Chapter III, Proposition 5]). This was pointed out by Colliot-Thélène, who also supplied the following direct argument, which is valid in arbitrary characteristic.

The assertion being birationally invariant (cf. [29, Proposition 1.1r]), we are reduced, as in Proposition 3.1, to the case where $Z$ is a smooth Del Pezzo surface of degree 6. Notation being as explained in $\S 5$, Pic $\bar{Z} \simeq \mathbb{Z}^{4}$ has a standard orthogonal basis $\left\{l_{0}, l_{1}, l_{2}, l_{3}\right\}$, in which the canonical class writes

$$
\omega=-3 l_{0}+l_{1}+l_{2}+l_{3} \text {. }
$$

AsSERTION 1. The only classes $\xi \in$ Pic $\bar{Z}$ for which $(\xi . \omega)=-2$ and $\left(\xi^{2}\right)=0$ are those of the form $\xi=l_{0}-l_{i}(i=1,2,3)$. 
Indeed, write $\xi=\sum_{i=0}^{3} \varepsilon_{i} n_{i} l_{i}$, with $n_{i} \geqslant 0$ and $\varepsilon_{i}= \pm 1$ for each $i \geqslant 0$. Then the conditions imposed on $\xi$ correspond to the following relations:

$$
\left\{\begin{array}{l}
-3 \varepsilon_{0} n_{0}-\sum_{i=1}^{3} \varepsilon_{i} n_{i}=-2, \\
n_{0}^{2}-\sum_{i=1}^{3} n_{i}^{2}=0
\end{array}\right.
$$

Applying the Schwarz inequality to the vectors $\varepsilon=\left(\varepsilon_{1}, \varepsilon_{2}, \varepsilon_{3}\right)$ and $\mathbf{n}=$ $\left(n_{1}, n_{2}, n_{3}\right)$, we obtain

$$
\left|3 \varepsilon_{0} n_{0}-2\right|=\left|\sum_{i=1}^{3} \varepsilon_{i} n_{i}\right| \leqslant(\sqrt{ } 3)\left(\sum_{i=1}^{3} n_{i}^{2}\right)^{\frac{1}{2}}=(\sqrt{ } 3) n_{0}
$$

The only solution of this inequality is $\varepsilon_{0}=+1, n_{0}=1$. Whence we see that $\xi$ is of the form indicated. A similar argument yields:

ASSERTION 2. The only classes $\xi \in \operatorname{Pic} \bar{Z}$ for which $(\xi . \omega)=-3$ and $\left(\xi^{2}\right)=1$ are $\xi=l_{0}$ and $\xi=2 l_{0}-l_{1}-l_{2}-l_{3}$.

Now, as $\mathscr{G}=\mathrm{Gal}(\bar{k} / k)$ preserves the intersection form and the canonical class, we see that the $\mathscr{G}$-module

$$
M=\operatorname{Pic} \bar{Z} \oplus \mathbb{Z} \xi,
$$

with trivial action on $\xi$, has the following $\mathcal{G}$-invariant basis:

$$
B=\left\{l_{0}-l_{1}-\xi, l_{0}-l_{2}-\xi, l_{0}-l_{3}-\xi, l_{0}-\xi, 2 l_{0}-l_{1}-l_{2}-l_{3}-\xi\right\} .
$$

To check that $B$ is indeed a basis, it is useful to observe that $l_{0}$ is the sum of the linear combination of the five vectors with coefficients $-1,-1,-1,2,1$.

(c) This is an immediate consequence of (b); see [46, Theorem 4.61].

(d) It follows from (b) that $H^{1}(k, S)=0$, where $S$ denotes the Néron-Severi torus of $Z$. Now, by a result of Colliot-Thélène [10, Proposition 7], the 'characteristic homomorphism' $\Phi: A_{0}(Z) \rightarrow H^{1}(k, S)$ is injective. Hence $A_{0}(Z)=0$.

Remark. None of the assertions of Proposition 3.2 holds for the class of cubic surfaces with two conjugate double points, as can be seen from Example 3.3 below. Indeed, by [46, Corollary 6.40], it would follow from (c) that $W^{1}(\mathbb{Q}, S)=0$, which is not the case (cf. Property (c) in the subsequent Remark). Neither is $A_{0}(Z)$ equal to zero. Hence none of the assertions of Proposition 3.2 holds for the singular cubic surface defined by (3).

EXAMPLE 3.3. The following varieties are birationally equivalent over $\mathbb{Q}$ : the conic bundle surface $V_{1} \subset \mathbb{A}_{Q}^{3}$ defined by

$$
Y^{2}-221 Z^{2}=\left(X^{2}-13\right)\left(X^{2}-17\right) \text {; }
$$

the singular intersection of two quadrics $V_{2} \subset \mathbb{P}_{Q}^{4}$ defined by

$$
\left\{\begin{array}{l}
x_{1}^{2}-221 x_{2}^{2}=x_{3}^{2}-4 x_{0} x_{3}, \\
x_{0} x_{3}=x_{4}^{2}-13 x_{0}^{2}
\end{array}\right.
$$


the singular cubic surface $V_{3} \subset \mathbb{P}_{Q}^{3}$ defined by

$$
t^{2}(30 x+15 y-2 z)+x\left(-221 x^{2}+y^{2}+z^{2}-15 y z\right)=0 ;
$$

the smooth intersection of two quadrics $U \subset \mathbb{P}_{Q}^{4}$ defined by

$$
\left\{\begin{array}{l}
\tau v=-221 \xi^{2}+\eta^{2}+\zeta^{2}-15 \eta \zeta \\
(30 \xi+15 \eta-2 \zeta) v+\xi \tau=0
\end{array}\right.
$$

the smooth cubic surface $W \subset \mathbb{P}_{\mathbb{Q}}^{3}$ defined by

(5) $1105 X\left(30 Y^{2}+2 Z^{2}-19 Y Z\right)+T\left(371 X^{2}+5 Y^{2}+5 Z^{2}-75 Y Z\right)+T^{2} X=0$.

Proof. The equivalence of (1) and (2) is obtained by putting

$$
\left(x_{1}, x_{2}, x_{3}, x_{4}\right)=\left(Y, Z, X^{2}-13, X\right) \text {, }
$$

and making the system (2) homogeneous.

The surface $V_{3}$ is obtained by projecting $V_{2}$ into $\mathbb{P}_{\mathbb{Q}}^{3}$ from the $\mathbb{Q}$-point $M=(0,15,1,2,0)$. (As $M$ does not lie on any line of $\bar{V}_{2}$, this is the same as blowing up $M$ on $V_{2}$.) So the transformation is determined by the choice of four generators of the linear system $\mathscr{L}$ of all hyperplanes passing through $M$. The particular choice made here is one for which the equation of $V_{3}$ becomes especially simple:

$$
(x, y, z, t)=\left(x_{0}, x_{1}-15 x_{2}, x_{3}-2 x_{2}+13 x_{0}, x_{4}\right) .
$$

One goes from (2) to (3) by writing $x_{1}=y+15 x_{2}$, etc., and eliminating $x_{2}$. Thus $x_{2}=\left(t^{2}-x z\right) / 2 x$. Note that $V_{3}$ contains the line $L_{0}$ defined by $t=x=0$, on which are the two double points $(0,15 \pm \sqrt{ } 221,2,0)$.

Then the equations (4) of an equivalent smooth Del Pezzo surface of degree 4 are given directly by Corollary 2.3 (cf. Remark (a) at the end of $\S 2$ ).

Finally, $V_{3}$ is certainly birationally equivalent to a smooth cubic surface $W$. But the point $P=(0,0,0,1)$ cannot be used for defining the transformation $\Phi$ of Theorem 2.2, since it lies on three lines of $\bar{V}_{3}$. In order to obtain the equation of $W$, it is therefore necessary to select some other $\mathbb{Q}$-point on $V_{3}$. A possible choice is $(5,11,-28,0)$. After the coordinate change $x=5 t^{\prime}, y=y^{\prime}+11 t^{\prime}, z=z^{\prime}-28 t^{\prime}$, $t=x^{\prime}$, the method of Theorem 2.2 yields equation (5) and shows that $W$ is smooth. (This does not follow immediately from Theorem 2.2 as stated, since the coordinate change we have used is not sufficiently general: with the notations of the theorem, this is a case in which $C \supset L_{0}$. However, it is not difficult to adapt the proof so as to include this special situation.)

Remark. This example, which appeared for the first time in [45], has numerous interesting properties. We list some of them without proof. For details, see $[38, \S \mathrm{V}]$ and $[29, \S 2]$. In what follows, $Z$ denotes any smooth projective surface which is birationally equivalent over $\mathbb{Q}$ to the surfaces of the example.

(a) $Z$ is $\mathbb{Q}$-unirational.

(b) For every completion $\mathbb{Q}_{v}$ of $\mathbb{Q}$, the surface $Z_{v}$ is $\mathbb{Q}_{v}$-rational. However, $Z$ is not $\mathbb{Q}$-rational.

(c) The Chow group $A_{0}(Z)$ does not embed in $\bigoplus_{v} A_{0}\left(Z_{v}\right)$. In fact $A_{0}(Z)=\mathbb{Z} / 2$, while $A_{0}\left(Z_{v}\right)=0$ for all $v$. Thus the kernel $\amalg A_{0}(Z)=$ $\operatorname{ker}\left(A_{0}(Z) \rightarrow \bigoplus_{v} A_{0}\left(Z_{v}\right)\right)$ is equal to $\mathbb{Z} / 2$ and coincides with the group $\Psi^{1}(\mathbb{Q}, S)=\operatorname{ker}\left(H^{1}(\mathbb{Q}, S) \rightarrow \bigoplus_{v} H^{1}\left(\mathbb{Q}_{v}, S\right)\right)$ associated with the Néron-Severi torus of $Z$. 
(d) $R$-equivalence is trivial everywhere locally, but not globally. In fact, $Z(\mathbb{Q}) / R=A_{0}(Z)=\mathbb{Z} / 2$, as follows from [15, Chapter VIII, Theorem 8].

(e) The Brauer group of $Z$ is non-trivial: $\operatorname{Br} Z / B r \mathbb{Q}=\mathbb{Z} / 2$. Nevertheless, Brauer equivalence is trivial on $Z$, locally and also globally since Brauer equivalence satisfies the Hasse principle. This is a case where Brauer equivalence is strictly coarser than $R$-equivalence.

(f) The weak approximation property holds for the Néron-Severi torus of $Z$ (cf. [29, Proposition 1.3]).

The principal interest of Example 3.3 is that it provides explicit equations of rational surfaces in various classes for which these phenomena occur.

Another interesting application of Theorem 2.2 is to the following proposition, which is used in [3] as a step towards proving that the Zariski problem has a negative solution for surfaces over a non-algebraically closed field.

Proposition 3.4. Let $f \in k[x]$ be an irreducible polynomial of degree 3. Suppose $\operatorname{char}(k) \neq 2$ and $d \in k$ is not a square. Then the 'generalized Châtelet surface' $V \subset \mathbb{A}_{k}^{3}$ with equation

$$
y^{2}-d z^{2}=f(x)
$$

is not $k$-rational.

Proof. We can replace $V$ by its compactification $V_{1} \subset \mathbb{P}_{k}^{3}$, with equation

$$
\left(y^{2}-d z^{2}\right) t=F(x, t),
$$

where $F$ is the cubic form defined by $F(x, 1)=f(x)$. This is a singular cubic surface, with two double points (of type $A_{2}$ ) on the line $t=x=0$. As we know, $V_{1}$ is birationally equivalent to a smooth cubic surface $W \subset \mathbb{P}_{k}^{3}$, which contains three $k$-rational lines (in the plane $\pi^{\prime}$ ) and a pair of conjugate lines (in the plane $\pi_{P}$ ). Before going into details, we give a brief sketch of the argument. It is possible to compute all the lines on $\bar{W}$ and to describe how the Galois group $\mathscr{G}=\mathrm{Gal}(\bar{k} / k)$ acts on them. Thus, using Theorem 2.2, we shall see that no set of 2, 3, or 6 mutually skew lines of $\bar{W}$ is invariant under the action of $\mathscr{G}$. By a result of Swinnerton-Dyer [43], this suffices to establish that $W$ is not $k$-rational. (Note that we need the full strength of Swinnerton-Dyer's result: the criterion discovered earlier by B. Segre is insufficient, since $W$ contains a $k$-rational line.) In what follows, we use the standard notation for the orbits of $\mathscr{G}$, as in Table 1 of [32, Chapter IV, § 9]. Thus, for example, the symbol $1^{2} 2^{1} 6^{2}$ means: two orbits of order 1 , one of order 2 , and two of order 6 . Since we need to apply the transformation $\Phi$ twice, we begin by describing all the lines of $V_{1}$, then those of its transform $V_{2}$ (which is of type $2 A_{1}$ ), and finally those of the smooth transform $W$ of $V_{2}$.

(a) $\bar{V}_{1}$ contains seven lines, forming two orbits for the action of $\mathscr{G}$ (symbol $\left.\mathbf{1}^{1} \mathbf{6}^{1}\right)$, namely:

$\left(\mathbf{1}^{1}\right)$ the line $L_{0}^{*}$, with equations $t=x=0$;

$\left(6^{1}\right)$ a pair of lines $D_{i, \lambda}^{*}(i=1,2)$ in each plane with equation $x=\lambda t$, where $\lambda$ is any root of $f$.

As $d$ is not a square, and $f$ is irreducible, we see that $\mathscr{G}$ acts transitively on the last 
six lines. Moreover, the intersection of $V_{1}$ with the plane $t=0$ consists only of the line $L_{0}^{*}$ (with multiplicity 3). From this it is evident that there are no other lines on $\bar{V}_{1}$. The situation is represented on Fig. 2.

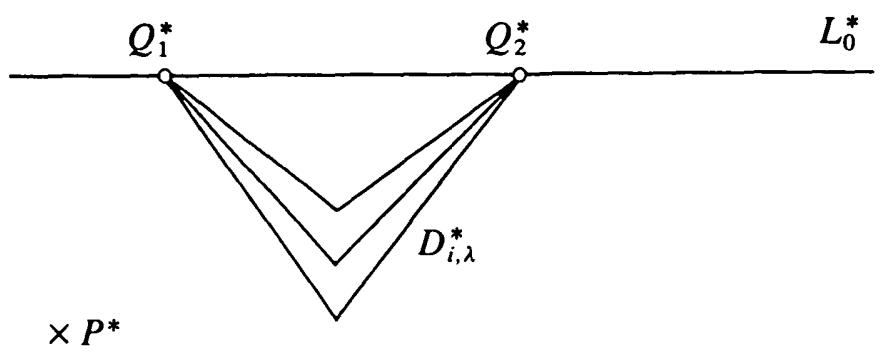

FIG. 2

(b) By Lemma 1.2, we can choose a suitable $k$-point $P^{*}$ on $V_{1}$, and a conic $C^{*}$ which define a transformation $\Phi^{*}$, as in Theorem 2.2. Let $V_{2}=\Phi^{*}\left[V_{1}\right]$, which is of type $2 A_{1}$. The lines on $\bar{V}_{2}$ form five orbits, which are described by the symbol $\mathbf{1}^{2} \mathbf{2}^{1} \mathbf{6}^{2}$. Indeed, $\bar{V}_{2}$ contains the following 16 lines:

$\left(\mathbf{1}^{2}\right)$ the blow-up $L_{P *}$ of $P^{*}$;

the line $L_{0}$ joining the two new double points, $Q_{1}$ and $Q_{2}$, which is also the image of the conic in the plane $\left\langle P^{*}, L_{0}^{*}\right\rangle$;

$\left(2^{1}\right)$ the 'blow-ups' (in the sense of Lemma 2.1(d)), $L_{1}^{*}$ and $L_{2}^{*}$, of $Q_{1}^{*}$ and $Q_{2}^{*}$;

$\left(6^{2}\right)$ the images $D_{i, \lambda}$ of the lines $D_{i, \lambda}^{*}$;

the image $E_{i, \lambda}$ of the conic $C_{i, \lambda}$ residual to $D_{i, \lambda}^{*}$ in the intersection of $\bar{V}_{1}$ with each plane $\left\langle P^{*}, D_{i, \lambda}^{*}\right\rangle$.

To check, for instance, that each $E_{i, \lambda}$ is a straight line, one computes its degree, i.e. its intersection number with a general plane. This is also the number of movable intersections of the corresponding conic $C_{i, \lambda}$ with a general quadric of the linear system associated with $\Phi^{*}$. Now, apart from $P^{*}$ and $Q_{i}^{*}$, there is one intersection on the conic $C^{*}$; so there is only one point left off the base locus of $\Phi^{*}$. Hence the degree is equal to 1 . The situation is depicted on Fig. 3, but not all incidence relations are shown (cf. the note at the end of $\S 1$ ).

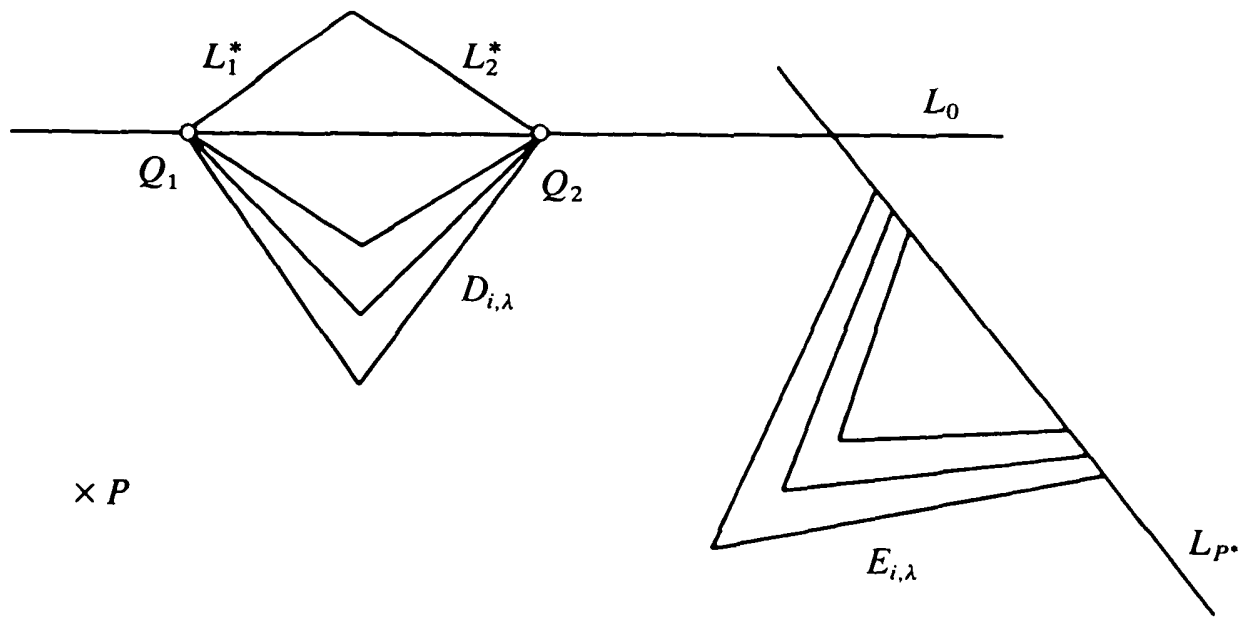

Fig. 3 
It can be shown that there are no other lines on $\bar{V}_{2}$ (cf. [6]). However, this is not needed for what follows, so we omit the proof.

(c) Now we choose a suitable $k$-point $P$ on $V_{2}$, and a conic $C$, which define a transformation $\Phi$, as in Theorem 2.2. Then $W=\Phi\left[V_{2}\right]$ is smooth. Therefore $\bar{W}$ contains 27 lines, which we proceed to display:

$\left(1^{3}\right)$ the blow-up $L_{P}$ of $P$;

the line $L^{\prime}$, which is the image of the conic in the plane $\left\langle P, L_{0}\right\rangle$;

the line $L^{\prime \prime}$, which is the image of the conic in the plane $\left\langle P, L_{P^{*}}\right\rangle$;

$\left(2^{3}\right)$ the 'blow-ups', $L_{1}$ and $L_{2}$, of $Q_{1}$ and $Q_{2}$;

the images of the lines $L_{1}^{*}$ and $L_{2}^{*}$;

the images of the conics in the planes $\left\langle P, L_{i}^{*}\right\rangle$;

$\left(6^{3}\right)$ the images of the lines $E_{i, \lambda}$;

the images of the lines $D_{i, \lambda}$;

the images of the conics in the planes $\left\langle P, D_{i, \lambda}\right\rangle$.

Hence there are altogether nine orbits, which are described by the symbol $1^{3} 2^{3} 6^{3}$. They have been enumerated in such a way that, for each order $(1,2$, or 6$)$, the lines in the first orbit meet $L^{\prime}$; those in the second orbit meet $L^{\prime \prime}$; finally those in the third orbit meet $L_{P}$. The above description makes it clear that no set of 2, 3, or 6 mutually skew lines on $\bar{W}$ can be invariant under the action of $\mathscr{G}$, which is all we had to prove.

\section{A discussion of the converse}

It is an exercise to show that the converse of Theorem 2.2 holds, in the following sense:

PRoposition 4.1. Every smooth cubic surface $W \subset \mathbb{P}_{k}^{3}$ with three coplanar $k$-rational lines and a further $k$-rational tritangent plane through one of them (like $\pi^{\prime}$ and $\pi_{P}$ in Fig. 1) is $k$-birationally equivalent to a cubic surface with two double points.

Proof. We label the various data as in Fig. 1: the given three $k$-rational lines are therefore denoted $L^{\prime}, L^{\prime \prime}, L_{P}$; the other known $k$-rational tritangent plane (through $L^{\prime}$ ) contains a pair of lines, $L_{1}$ and $L_{2}$, lying on $\bar{W}$, which meet in a $k$-point; call it $P$. We choose a $k$-rational plane $\pi \neq \pi^{\prime}$ through $L_{P}$, such that $P \notin \pi$ and $\pi \cap W=L_{P} \cup C$, where $C$ is a conic. (Clearly, we could assume that $C$ is smooth, but this assumption is unnecessary. In fact, allowing $C$ to be degenerate sometimes leads to easier computations.)

Now consider the quadratic transformation $\Phi$ defined by $C$ and $P$. It is easy to verify that the proper transform $V=\Phi[W]$ is a cubic surface. (The argument is identical with that used in Part (a) of the proof of Theorem 2.2.) By Lemma 2.1(c), the lines $L_{1}$ and $L_{2}$ are blown down into two points $Q_{1}$ and $Q_{2}$ on $\bar{V}$. To check that $Q_{1}$ is a double point of $\bar{V}$, we make the following observation: a general line $\widetilde{L}$ through $Q_{1}$ is the image of the intersection of two general quadrics (in the linear system $\mathcal{M}$ ) containing $L_{1}$. This intersection is of the form $C \cup L_{1} \cup L$, where $L$ is a general line meeting both $C$ and $L_{1}$. Thus $L$ meets $\bar{W}$ in one movable point, off $C \cup L_{1}$. This means that $\tilde{L}=\Phi[L]$ meets $\bar{V}$ in one point other than $Q_{1}$, whence $Q_{1}$ is double. It can be seen, by a direct argument or by 
applying the Zariski Main Theorem, that $\bar{V}$ has no singularities other than $Q_{1}$ and $Q_{2}$.

Scholium. The above argument is completely general. But it includes a particularly noteworthy situation, which does not appear clearly on Fig. 1: this is the case in which $P$ is an Eckardt point, that is, $P \in L^{\prime}$. When this happens, the image $\Phi\left[L^{\prime}\right]$ of the line $L^{\prime}$-instead of being a smooth conic (in the plane $\left.\left\langle P, L_{0}\right\rangle\right)$ as in the general case (see Part (c) of the proof of Proposition 3.4)-is the line denoted $L_{P^{*}}$ in Fig. 3. (This is characterized by the fact that the tangent plane to $V$ at any smooth point on $L_{0}$ meets $V$ in the cycle $2 L_{0}+L_{P^{*}}$.) Moreover, it is easily seen that $P$ lies on $L_{P *} \backslash L_{0}$.

With the notation used in the proof of Theorem 2.2 (the equations of $L^{\prime}$ were given in Part (c)), this special situation is one in which $g=0$. In $\S 2$, the inequality $g \neq 0$ occurred in (7) and was used later to prove the smoothness of $W$. As a matter of fact, the reader can check that $W$ is also smooth when $g=0$, though the proof is slightly more complicated. (Of course one must still assume that $P$ does not lie on any line other than $L_{P^{*}}$. Otherwise, a counter-example is provided by the surface $V_{3}$ of Example 3.3, where $P$ is an Eckardt point!) A consequence of this discussion is that, by choosing $P$ on $L_{P^{*}}$, we can arrange for the smooth cubic surface $W$ of Theorem 2.2 to contain a $k$-rational Eckardt point on one of the three $k$-rational lines!

Remark. Swinnerton-Dyer, in a letter to Colliot-Thélène, furnished an elementary argument by which a cubic surface $V$ of type $2 A_{1}$ can be transformed into one of type $2 A_{2}$, provided some arithmetic condition is satisfied. For simplicity we assume that char $k \neq 2$. Then the equation of $V$ can be brought to the form

$$
\left(Y^{2}-d Z^{2}\right) T+2 Y \beta_{1}(X, T)+2 Z \beta_{2}(X, T)+\gamma(X, T)=0,
$$

where the $\beta_{i}$ are quadratic, and $\gamma$ is cubic. Now we set $T=1$ and define

$$
x=X, \quad y=Y+\beta_{1}(X, 1), \quad z=Z-\frac{1}{d} \beta_{2}(X, 1) .
$$

This transforms $V$ into the conic bundle surface $V^{\prime} \subset \mathbb{A}_{k}^{3}$ with equation

$$
y^{2}-d z^{2}=g(x),
$$

where $g \in k[x]$ is a polynomial of degree 4. If $g$ has a root in the field $k$, we can send it to infinity by an automorphism of $\mathbb{P}_{k}^{1}$. Then $g$ is replaced by a polynomial $f$ of degree 3, and we obtain a cubic, which is a generalized Châtelet surface (as in Proposition 3.4). We may add that the quadratic transformation defined by (2) is of a very degenerate type (cf. the Remark before Lemma 2.1): $C$ is the double line $X^{2}=0$ in the plane $T=0$, and $P=(0,1,0,0)$ is a point of osculation on the line $X=T=0$. (See [22, p. 198, end of $\S 17]$.)

In view of these results, it is natural to ask whether some weaker assumption might suffice to prove the converse. The next proposition shows that having one, or even three, $k$-rational lines is not a sufficient condition. We denote by $\mathscr{D}_{h}$ the family of all cubic surfaces in $\mathbb{P}_{k}^{3}$ which are birationally equivalent to a singular 
cubic surface with a $k$-rational set of $h$ double points. Further we denote by $\mathscr{L}_{j}$ the family of all cubic surfaces in $\mathbb{P}_{k}^{3}$ which are birationally equivalent to a smooth cubic surface with $j$ lines individually defined over $k$. Finally, let $\mathscr{R}$ be the family of all $k$-rational cubic surfaces in $\mathbb{P}_{k}^{3}$. Then, by Theorem $2.2, \mathscr{D}_{2} \subset \mathscr{L}_{3}$, so that the following inclusions hold:

$$
\mathscr{R} \subset \mathscr{D}_{2} \subset \mathscr{L}_{3} \subset \mathscr{L}_{1} .
$$

Note that $\mathscr{L}_{3}$ is the same as $\mathscr{L}_{2}$. Indeed, if two $k$-rational lines on a smooth cubic surface are skew then the surface is in $\mathscr{R}$. If they meet, then there is a third line in their plane; so the surface is in $\mathscr{L}_{3}$. On the other hand, by Proposition 3.4 we know that, for most fields, $\mathscr{R} \neq \mathscr{D}_{2}$.

Remark. If $k=\mathbb{R}$ then $\mathscr{R} \neq \mathscr{D}_{2}=\mathscr{L}_{3}=\mathscr{L}_{1}$. Indeed, it is well known that every smooth cubic surface $W$ over $\mathbb{R}$ contains at least three coplanar real lines. (Hint. There is at least one real line $l$. Among all the lines which are skew to $l$, we can find a pair $\left\{d_{1}, d_{2}\right\}$ defined over $\mathbb{R}$. If $d_{1} \cap d_{2} \neq \varnothing$, then the third line in the plane $\left\langle d_{1}, d_{2}\right\rangle$ is a real line that meets $l$. If $d_{1} \cap d_{2}=\varnothing$ then $T=\left\{l, d_{1}, d_{2}\right\}$ is a skew triplet defined over $\mathbb{R}$. Consider the complementary triplet (in the intersection of $W$ with the unique quadric through $T$; cf. [17, Remark 1])!) That $\mathscr{L}_{3}=\mathscr{D}_{2}$ follows from Proposition 4.1. (Hint. If there is no further real tritangent plane through $l$, then $W$ contains a pair of skew lines, and hence it is already in $\mathscr{R}$.) Finally $\mathscr{D}_{2} \neq \mathscr{R}$. Otherwise, by what we have seen so far, all smooth cubic surfaces would be birationally trivial over $\mathbb{R}$, and we know this is not the case: those having two real components are not birationally trivial.

Proposition 4.2. If $k$ is a finite field, then all three inclusions in (4) are strict: $\mathscr{R} \neq \mathscr{D}_{2} \neq \mathscr{L}_{3} \neq \mathscr{L}_{1}$.

Proof (communicated by Swinnerton-Dyer). We start from the following idea: over any field $k$, if $V \subset \mathbb{P}_{k}^{3}$ is a cubic surface with two double points, each of them is defined over a quadratic extension $K / k$; hence $V_{K}$ is $K$-rational. Thus, in order to show that a surface $W \in \mathscr{L}_{1}$ is not in $\mathscr{D}_{2}$, it suffices to prove that no quadratic extension $K / k$ has the property that $W_{K}$ is $K$-rational. Now this is particularly easy to do over a finite field, since there is a unique quadratic extension. Moreover, we possess an extensive classification of cubic surfaces over finite fields ([42]; see also [32, Chapter IV, §9]). Thus the argument will be based on Table 1 of [42], and its subdivision into 25 classes $c_{i}$.

To begin with, we make the following remark, which derives from that table: if a cubic surface $W$, defined over a finite field $k=\mathbb{F}_{q}$, contains a $k$-rational line, but is not $k$-rational, then $W$ belongs to one of the classes $c_{3}, c_{4}, c_{7}, c_{19}, c_{20}$, or $c_{24}$. (In all other cases where $W$ contains a $k$-rational line, one can see from the third column (and Table 2 in the case $c_{21}$ ) that $W$ contains a $k$-rational pair of skew lines; hence it is $k$-rational.) Now we use the last column of Table 1 , which contains the orders of the roots of unity $\eta_{i}=\omega_{i} / q$, where the $\omega_{i}$ are the eigenvalues of the Frobenius endomorphism acting on Pic $\bar{W}$. Since going over to the quadratic extension $K=\mathbb{F}_{q^{2}}$ of $\mathbb{F}_{q}$ replaces $\omega_{i}$ by $\omega_{i}^{2}$, we see, for instance, that class $c_{24}$ (where the orders are $1,2,4,4,6,6$ ) goes over to a class whose orders 
are $1,1,2,2,3,3$, namely $c_{8}$. In this way we get the following table:

\begin{tabular}{l|lllrrr}
\hline Class over $k=\mathbb{F}_{q}$ & 3 & 4 & 7 & 19 & 20 & 24 \\
Class over $K=\mathbb{F}_{q^{2}}$ & 1 & 3 & 6 & 2 & 4 & 8 \\
\hline
\end{tabular}

In particular, classes $c_{3}, c_{7}, c_{19}$, and $c_{24}$ become rational over $K$, whereas $c_{4}$ and $c_{20}$ do not. Indeed it follows from [43], or from column 7 of Table 1 in [32, Chapter IV, $\S 9$ ], that a surface in class $c_{3}$ is not $k$-rational. $A$ fortiori a surface in class $c_{4}$ is not $k$-rational.

Now suppose we take a surface $W \subset \mathbb{P}_{k}^{3}$ in class $c_{3}$. From Table 1 of [42] we see that $W$ contains three coplanar $k$-rational lines and a further $k$-rational tritangent

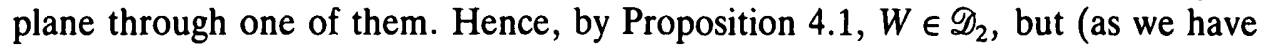
just remarked) $W \notin R$. (Alternatively, take a surface $V$ given by equation (6) of $\S 3$. The proof of Proposition 3.4 shows that $V \in \mathscr{D}_{2} \backslash \mathscr{R}$, and also that $V$ is equivalent to a smooth surface $W$ in class $c_{7}$.)

If we start from a surface $W$ in class $c_{4}$, we see from Table 1 of [42] that $W \in \mathscr{L}_{3}$. But $W \notin \mathscr{D}_{2}$; indeed $W_{K}$ is not $K$-rational, since it belongs to class $c_{3}$.

Finally, let us take a surface $W$ in class $c_{20}$. Then $W \in \mathscr{L}_{1}$, but $W \notin \mathscr{L}_{3}$. Indeed, suppose $W$ is birationally equivalent to a smooth surface $V$ with three $k$-rational lines. Then (since we know already that $W \notin \mathscr{R}) V$ is in one of the classes $c_{3}, c_{4}$, or $c_{7}$. Therefore, if we denote by $L$ the biquadratic extension $L=\mathbb{F}_{q}{ }^{4}$, we see from the above table that $V_{L}$ is birationally trivial. However, this is impossible, since $W_{L}$ is only in class $c_{3}$.

What we have proved so far is that the proposition holds over a finite field $k$, provided there exists a surface in each class as required (viz. $c_{3}, c_{4}$, or $c_{20}$, as the case may be). In a letter to the authors, Swinnerton-Dyer asserts that all 25 classes do exist over any finite field. What is more, one can use the methods of [42] to work out the exact number of surfaces of each type over $\mathbb{F}_{q}$, 'though the calculation is extremely tedious'! We shall content ourselves with a weaker result: we show that, for any given class $c_{v}$, there exists a cubic surface in this class over some finite field. The argument that follows was also suggested by SwinnertonDyer.

If one considers the generic cubic surface over a number field $F$ (which is therefore defined over a transcendental extension $\Omega$ of $F$ ), then the degree $\left[\Omega_{1}: \Omega\right]$ of the least field $\Omega_{1}$ over which the 27 lines are defined, is equal to the order of the group $G$ of those permutations of the 27 lines which preserve (pairwise) incidence relations (cf. [32, Chapter IV, §1.12]). It follows from Hilbert's irreducibility theorem that, for most cubic surfaces over $F$, the Galois group of the field of definition of the 27 lines is the full group $G$. Take one surface $W$ for which this is true. By Cebotarev's density theorem, every conjugacy class $c_{v}$ in $G$ is the class of the Frobenius automorphism for an infinity of primes. Thus, when we reduce modulo one of those primes, we get a cubic surface over a finite field $\mathbb{F}_{q}$ which is in the required class $c_{v}$.

In the course of the proof, we have obtained the following result, which provides an answer to a question raised in [11, Remark 6.7(i)]. 
Proposition 4.3. There exists a cubic surface $W$, defined over a finite field $k=\mathbb{F}_{q}$, which has a $k$-rational line and becomes birationally trivial over $\mathbb{F}_{q^{8}}$, but over no intermediate extension.

Proof. Indeed we have seen that a surface in class $c_{20}$ is in class $c_{4}$ over $\mathbb{F}_{q^{2}}$, in class $c_{3}$ over $\mathbb{F}_{q^{4}}$, and becomes rational only over $\mathbb{F}_{q}$.

REMARK. The question in [11] was motivated by a study of torsion in the Chow group $A_{0}(W)=\operatorname{ker}\left(C_{0}(W) \rightarrow \mathbb{Z}\right)$. It is therefore worth noting that here $A_{0}(W)=0$. Indeed, it is a result of Bloch that, over a finite field $k$, this group is trivial for any smooth projective variety which becomes rational over $\bar{k}$.

The reader may want to see some more explicit example of a variety in $\mathscr{L}_{1} \backslash \mathscr{D}_{2}$, over a number field, say. It is difficult to use Proposition 4.2 for that purpose, for if we lift a surface in class $c_{20}$, it may be birationally equivalent over $\mathbb{Q}$ to one in class $\mathscr{L}_{3}$ with a bad reduction! Furthermore, even in the case of good reduction, birational equivalence does not seem to behave well under reduction. Fortunately, Colliot-Thélène has shown us a particularly interesting collection of examples, of a type which does not exist over a finite field.

Example 4.4.1 (Colliot-Thélène). Let $W \subset \mathbb{P}_{k}^{3}$ be the smooth cubic surface defined by

$$
\left(x-e_{1} t\right)\left(x-e_{2} t\right)\left(x-e_{3} t\right)+\left(x-e_{4} t\right) y^{2}+\left(x-e_{5} t\right) z^{2}=0,
$$

with $e_{i} \in k(i=1, \ldots, 5)$ and $6 \prod_{i \neq j}\left(e_{i}-e_{j}\right) \neq 0$. Then $W \in \mathscr{L}_{1} \backslash \mathscr{L}_{3}$, providing none of the following numbers is a square in $k$ :

$$
\frac{e_{i}-e_{4}}{e_{5}-e_{i}}(i=1,2,3) ; \frac{\prod_{i=1}^{3}\left(e_{4}-e_{i}\right)}{e_{5}-e_{4}} ; \frac{\prod_{i=1}^{3}\left(e_{5}-e_{i}\right)}{e_{4}-e_{5}}
$$

(For instance, if $k=\mathbb{Q}$, the conditions are fulfilled if we take $e_{i}=i$.)

Proof. The surface $W$ is a conic bundle surface with invariant 5 , the degenerate fibres corresponding to $x / t=e_{i}(i=1, \ldots, 5)$. Moreover, $W$ contains the line $E$ with equations $x=t=0$, and the conditions guarantee that every line $L$ on $\bar{W}$ which meets $\bar{E}$ is conjugate to the residual line in the plane spanned by $L$ and $\bar{E}$. This ensures that $W \rightarrow \mathbb{P}_{k}^{1}$ is a relatively minimal conic bundle. Now suppose $W$ were birationally equivalent to a smooth cubic surface with three $k$-rational lines say $D, D^{\prime}, D^{\prime \prime}$. On considering the pencil of planes through $D$, we could view this surface as a conic bundle with invariant 5 . Then we could blow down one of the lines $D^{\prime}$ or $D^{\prime \prime}$ (and perhaps some further exceptional divisors of the first kind in the fibres), so as to obtain a relatively minimal conic bundle surface $V \rightarrow \mathbb{P}_{k}^{1}$ with invariant $r \leqslant 4$. It follows from a result of Iskovskih [23, Theorem 2.5, plus the argument of Corollary 1.7] that there would exist a fibre-preserving birational equivalence between $V$ and $W$. But this is impossible, since the invariants differ (see, for example, [7, Theorem 4.11]).

An example in $\mathscr{L}_{3} \mid \mathscr{D}_{2}$ has been found by Kunjavskĩ. He starts from the 
equation

$$
y^{2}-x z^{2}=\left(x^{2}-a\right)\left(x^{2}-a^{3}\right),
$$

which defines a (non-minimal) conic bundle surface $Y$ over the affine line Spec $\mathbb{Q}[x]$. We assume that $a \neq-1$ and is not a square in $\mathbb{Q}$. The degenerate fibres are those above $x= \pm \sqrt{ } a, \pm \sqrt{ } a^{3}, 0$, and $\infty$. Whence we see that the splitting field of $Y$ is the extension $\mathbb{Q}(i, \sqrt[4]{ } a) / \mathbb{Q}$, whose Galois group $G$ is the dihedral group with eight elements. As in [29], we can view $G$ as a subgroup of the Weyl group associated with the Dynkin diagram $D_{5}$ (cf. [28, beginning of $\S 6])$. Then the three index-2 subgroups of $G$ are:

$\left\langle c_{2} c_{3}(12)(34)\right\rangle$, cyclic, corresponding to $\mathbb{Q}(i)$;

$\left\langle c_{2} c_{3}, c_{1} c_{4}\right\rangle$ and $\left\langle c_{1} c_{2} c_{3} c_{4},(12)(34)\right\rangle$, isomorphic to $\mathbb{Z} / 2 \times \mathbb{Z} / 2$, corresponding to $\mathbb{Q}(\sqrt{ } a)$ and $\mathbb{Q}(\sqrt{ }-a)$, respectively.

All of them contain the group $H=\left\langle c_{1} c_{2} c_{3} c_{4}\right\rangle$. Since the orbit decomposition for this group action is of Type IV (see [32, Chapter IV, $\S 9$, Table 2]), it follows from [32, loc. cit., Table 3], that

$$
H^{1}(H, \operatorname{Pic} \bar{Y})=\mathbb{Z} / 2 \times \mathbb{Z} / 2 \text {. }
$$

As a result, $Y$ does not become rational over any quadratic extension of $\mathbb{Q}$. Otherwise it would a fortiori become rational over the fixed field of $H$, and $H^{1}(H$, Pic $\bar{Y})$ would vanish.

Of course $Y$ is not a cubic surface, but it can easily be carried into one. Indeed (6) can also be written as follows:

$$
y^{2}-a^{4}=x\left(x^{3}-\left(a+a^{3}\right) x+z^{2}\right) .
$$

Hence it suffices to set $y-a^{2}=u x$ to obtain the birationally equivalent cubic surface with equation:

$$
u\left(u x+2 a^{2} t^{2}\right)=x^{3}-\left(a+a^{3}\right) x t^{2}+z^{2} t .
$$

EXAMPLe 4.4.2 (Kunjavskĩ). The cubic surface $W \subset \mathbb{P}_{Q}^{3}$ defined by (7) is smooth, and $W \in \mathscr{L}_{3} \backslash \mathscr{D}_{2}$.

Proof. One checks that $W$ is smooth and contains the union of three lines $\{t=0 ; x(x-u)(x+u)=0\}$. But $W \notin \mathscr{D}_{2}$ since, as we have seen for $Y$, it does not become rational over any quadratic extension of $\mathbb{Q}$.

Finally, we discuss the converse of Corollary 2.3 , which is extremely simple:

PRoposition 4.5. Every smooth intersection of two quadrics $U \subset \mathbb{P}_{k}^{4}$ with a $k$-rational pair of lines, $\tilde{L}_{1}$ and $\tilde{L}_{2}$, meeting in a point $\tilde{P}$, is $k$-birationally equivalent to a cubic surface with two double points.

Proof. It suffices to project $U$ from $\tilde{P}$ into $\mathbb{P}_{k}^{3}$. The general fibre of this projection $r: U \ldots \mathbb{P}_{k}^{3}$ is finite and consists of just one point (with multiplicity 1): if it contained more than one, say $P_{1}$ and $P_{2}$, then the line through $\widetilde{P}, P_{1}$, and $P_{2}$ would be entirely contained in each of the two quadrics (call them $Y_{1}$ and $Y_{2}$ ), and hence also in $U=Y_{1} \cap Y_{2}$. This is impossible, since $\widetilde{P}$ is smooth.

Therefore the image $V=r[U]$ is a cubic surface, which contains the two points 
$Q_{i}=r\left[\tilde{L}_{i}\right](i=1,2)$. It is an easy matter to check that the $Q_{i}$ are double on $\bar{V}$ : take a generic line $L$ through $Q_{1}$; lift it to a 2-plane in $\mathbb{P}^{4}$, which meets each of the two quadrics $Y_{i}$ in the union of $\tilde{L}_{1}$ and one other line $D_{i}$. Now $D_{1} \neq D_{2}$; or else $U$ would contain infinitely many lines. Hence $D_{1}$ and $D_{2}$ meet in only one point $R$, which lies off $\tilde{L}_{1}$. (Otherwise, a generic 2-plane through $\tilde{L}_{1}$ would meet $U$ only in $\tilde{L}_{1}$ !) Thus, apart from $Q_{1}$, the only intersection of $L$ with $\bar{V}$ is $r(R)$, whence $Q_{1}$ is double.

In the case where $\tilde{P}=(0,0,0,1,0)$, we may add that the projection $r$ maps $(\xi, \eta, \zeta, \tau, v)$ into $(\xi, \eta, \zeta, v)$. This is obviously a retraction (and hence the inverse) of the map $\Psi$ of Corollary 2.3 .

As an application of these results, we consider the surface $U \subset \mathbb{P}_{k}^{4}$ studied by Coombes and Muder in [16]. This is the intersection of quadrics defined by the system

$$
\left\{\begin{array}{l}
a\left(d \xi^{2}-\zeta^{2}\right)=(\tau+\eta)(\eta+v) \\
c\left(d \xi^{2}-\eta^{2}\right)=(\tau+\zeta)(\zeta-v)
\end{array}\right.
$$

where $a, c, d \in k^{*}$ (and $k$ is any field of characteristic not equal to 2). The point $\tilde{P}$ with coordinates $(\xi, \eta, \zeta, \tau, v)=(0,0,0,1,0)$ lies on the two lines defined by $v=\zeta=-\eta= \pm(\sqrt{ } d) \xi$. Therefore, by Proposition $4.5, U$ is birationally equivalent to a cubic surface $V$ with two double points. Hence, by the Remark following Proposition 4.1, $U$ is also birationally equivalent to a generalized Châtelet surface $V^{\prime}$, whose equation is of the form (3). As a matter of fact, the explicit computation reveals that $V^{\prime}$ is a true Châtelet surface. The necessary coordinate changes will appear quite natural if, instead of applying SwinnertonDyer's method, we use the geometric argument already employed in the proof of Proposition 4.1. For this reason we go into the details of the computation.

Proposition 4.6. The surface $U \subset \mathbb{P}_{k}^{4}$ defined by the system (8) is $k$-birationally equivalent to the Châtelet surface $V^{\prime} \subset \mathbb{A}_{k}^{3}$ with equation

$$
y^{2}-d z^{2}=x(x-a c)(x-(a+c-1)) .
$$

Proof. Projecting $U$ from $\tilde{P}$, which amounts to eliminating $\tau$ from the system (8), we obtain the following equation for $V$ :

$$
a\left(d \xi^{2}-\zeta^{2}\right)(\zeta-v)=\left\{c\left(d \xi^{2}-\eta^{2}\right)+(\eta-\zeta)(\zeta-v)\right\}(\eta+v) .
$$

This surface contains two double points on the line

$$
L_{0}=\{\eta+v=\zeta-v=0\} .
$$

The tangent plane $\pi^{\prime}$ to $V$ at a general point of $L_{0}$ is given by

$$
a(\zeta-v)-c(\eta+v)=0 .
$$

Hence the line denoted $L_{P^{*}}$ in Fig. 3 is given by

$$
\eta-\zeta=(a-c) \eta-(a+c) v=0 .
$$

With the notation used in the proof of Proposition 4.1, we can consider that we are in the degenerate case where $L^{\prime}=L^{\prime \prime}=L_{0}$, and set $L_{P}=L_{P^{*}}$.

One of the five tritangent planes through $L^{\prime}$ is the plane $\pi_{P}=\{\zeta-v=0\}$, 
whose intersection with $V$ contains the pair of lines $L_{1} \cup L_{2}=\left\{\zeta-v=d \xi^{2}-\eta^{2}=0\right\}$. These two lines meet in $P=(0,0,1,1)$. Finally, we must choose a $k$-rational plane $\pi \neq \pi^{\prime}$ through $L_{P}$. As the residual conic $C$ is not required to be irreducible, a convenient choice is $\pi=\{\eta-\zeta=0\}$. Therefore we define $T=\eta-\zeta, X=\zeta-v$, $Y=\xi, Z=\eta$. The point $P$ then becomes $(X, Y, Z, T)=(0,0,0,1)$, and the equation of $V$ reads

$$
\begin{aligned}
& \left(d Y^{2}-Z^{2}\right)\{(a+c) X-2 c Z\} \\
& +T\left\{2(a-1) X Z+X^{2}+c\left(d Y^{2}-Z^{2}\right)\right\}+(1-a) T^{2} X=0
\end{aligned}
$$

This is of the form (3) of $\S 2$. Thus $\Phi$ carries $V$ into the surface $V^{\prime}$ with equation (corresponding to equation (2) of $\S 2$ ):

$$
\begin{aligned}
t^{2}\{(a+c) x-2 c z\}+t\left\{2(a-1) x z+x^{2}+c\left(d y^{2}-z^{2}\right)\right\} & \\
& +(1-a) x\left(d y^{2}-z^{2}\right)=0 .
\end{aligned}
$$

If this is a Châtelet surface, one of the planes through $L_{0}^{*}=\{t=x=0\}$ meets $V^{\prime}$ along this line with multiplicity 3 . And indeed the plane with equation $x+(c /(1-a)) t=0$ has this property. We therefore define

$$
u=x+\frac{c}{1-a} t .
$$

The equation of $V^{\prime}$ now becomes

$$
(1-a) u\left(d y^{2}-z^{2}-2 t z\right)+t^{3} \frac{a c(a+c-1)}{(a-1)^{2}}+t^{2} u \frac{a(a+c-1)+c}{a-1}+t u^{2}=0 .
$$

We get rid of the term in $u t z$ by setting $v=z+t$. In addition we write $w=t /(1-a)$. This yields the equation

$$
\left(v^{2}-d y^{2}\right) u=w((a+c-1) w-u)(a c w-u) .
$$

Let $h=a c(a+c-1)$; it suffices to put $x=h w, y=h v, z=h y$, and $u=1$ to obtain the equation of $V^{\prime}$ in the form (9).

Proposition 4.6 can be used to determine the Chow group $A_{0}(U)$. Indeed, we know (cf. [11, Proposition 6.3]) that the functor $A_{0}$ is a birational invariant for smooth projective surfaces. So we can replace $U$ by any other smooth projective model of the Châtelet surface $V^{\prime}$, for instance, the one used in [13, § IV]. This yields in particular a very simple proof of the main result of [16, Theorems 4.4 , 4.5], which can be stated also more simply in terms of Châtelet surfaces. In this form, the result (communicated by Colliot-Thélène) is as follows:

Proposition 4.7 (Colliot-Thélène). Let $k$ be a local field of characteristic 0 and residual characteristic not equal to 2 , with discrete valuation $v$. Suppose $d$ is a non-square unit in $k, a_{1} a_{2}\left(a_{1}-a_{2}\right) \neq 0$, and $v\left(a_{1}\right)=0$ or $1, v\left(a_{2}\right) \geqslant 0$. Then the Chow group $A_{0}(X)$ of any smooth projective model $X$ of the Chatelet surface $Y$ with equation

$$
y^{2}-d z^{2}=x\left(x-a_{1}\right)\left(x-a_{2}\right)
$$


is equal to:

0

$\mathbb{Z} / 2$

if $Y$ has good reduction (that is, $v\left(a_{1}\right)=v\left(a_{2}\right)=v\left(a_{1}-a_{2}\right)=0$ );

if $Y$ has very bad reduction (that is, $v\left(a_{1}\right)=1, v\left(a_{2}\right) \geqslant 1$ );

in the remaining cases of 'not so bad' reduction.

Proof. As mentioned before, we are free to assume that $X$ is the standard model used in [13]. Let $O$ be the singular point of the fibre at infinity. We consider the commutative diagram

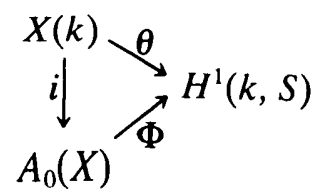

where $i$ is the map sending a $k$-point $P$ into the class of the 0 -cycle $P-O$, and $\Phi$ is the characteristic homomorphism defined in [14]. It is known that $i$ is surjective [11, Theorem C], and $\Phi$ injective [14, Theorem 2]. Therefore computing $A_{0}(X)$ is the same as computing the image of $\Phi$, which coincides with the image of $\theta$. Now, as explained in $[\mathbf{1 3}, \S \mathrm{IV}]$ and in [38, $\S$ III] there is an explicit formula for $\theta$ on some non-empty open subset $U$ of $X$, namely:

$$
\left.\theta\right|_{U}: P \mapsto\left(x(P), x(P)-a_{1}\right) \in\left(k^{*} / N K^{*}\right)^{2},
$$

where $K=k(\sqrt{ } d)$. In fact, $\theta$ is completely determined by its restriction to $U(k)$, but it will also be convenient to know how to compute directly its value at a $k$-point of $X \backslash U$. This is done as follows: suppose, for instance, we wish to compute $\theta$ at a point $P$ where $x(P)=0$. Then we can replace the first coordinate in (15) by $\left(x(P)-a_{1}\right)\left(x(P)-a_{2}\right)$, since $\left(x-a_{1}\right)\left(x-a_{2}\right)$ differs from $x$ by an element of $N_{K / k}\left(K(X)^{*}\right)$. This is a consequence of the 'general lemma' which appears on page 18 of [38]. In addition, the set $X(k) \backslash U(k)$ contains only the three points $P_{0}, P_{1}$, and $P_{\infty}$, which lie above $x=0, a_{1}$, and $\infty$, respectively. This enables us to compute the group $\operatorname{Im} \theta$ quite easily.

To begin with we list the images of the three exceptional points in $\left(k^{*} / N K^{*}\right)^{2}$ :

$$
\begin{aligned}
& \theta\left(P_{0}\right)=\left(a_{1} a_{2},-a_{1}\right), \\
& \theta\left(P_{1}\right)=\left(a_{1}, a_{1}\left(a_{1}-a_{2}\right)\right), \\
& \theta\left(P_{\infty}\right)=(1,1) .
\end{aligned}
$$

In what follows we use the additive notation and denote by 1 the non-trivial element of $\mathbb{Z} / 2$. Thus, in particular, $\theta\left(P_{\infty}\right)=(0,0)$. Note, further, that $x \in N K^{*}$ if and only if $v(x)$ is even.

First case: $v\left(a_{1}\right)=0$. In this case the group $\operatorname{Im} \theta$ cannot be $\mathbb{Z} / 2 \times \mathbb{Z} / 2$, since it does not contain the element $(1,1)$. Indeed, $(1,1)$ is not the image of any of the three exceptional points and, for $P \in U(k)$, we have

$$
v(x(P)) \text { odd } \Rightarrow v(x(P)) \geqslant 1 \text { and } v\left(x(P)-a_{1}\right)=0 .
$$

Hence it suffices to determine whether $\operatorname{Im} \theta$ is trivial or isomorphic to $\mathbb{Z} / 2$. We distinguish three subcases. 
(1) If $v\left(a_{2}\right) \geqslant 2$, we denote by $\pi$ an element of $k$ with valuation 1 . Then, by (14), there is a point $P_{\pi} \in U(k)$ such that $x\left(P_{\pi}\right)=\pi$. Now $\theta\left(P_{\pi}\right)=(1,0)$, whence $\operatorname{Im} \theta \simeq \mathbb{Z} / 2$.

(2) If $v\left(a_{2}\right)=1$, then $\theta\left(P_{0}\right)=(1,0)$, whence $\operatorname{Im} \theta \simeq \mathbb{Z} / 2$.

(3) If $v\left(a_{2}\right)=0$, we consider three subcases.

(3a) If $v\left(a_{1}-a_{2}\right) \geqslant 2$, there is a point $P^{\prime} \in U(k)$ such that $x\left(P^{\prime}\right)=\pi+a_{1}$. Now $\theta\left(P^{\prime}\right)=(0,1)$, whence $\operatorname{Im} \theta \simeq \mathbb{Z} / 2$.

(3b) If $v\left(a_{1}-a_{2}\right)=1$, then $\theta\left(P_{1}\right)=(0,1)$, whence $\operatorname{Im} \theta \simeq \mathbb{Z} / 2$.

(3c) Finally, if $v\left(a_{1}-a_{2}\right)=0$ then $\operatorname{Im} \theta$ is trivial. Indeed the three exceptional points do not contribute and, for $P \in U(k)$, we have

$$
v(x(P)) \text { odd } \Rightarrow v\left(x(P)-a_{1}\right)=v\left(x(P)-a_{2}\right)=0,
$$

which contradicts (14). Further,

$$
v\left(x(P)-a_{1}\right) \text { odd } \Rightarrow v(x(P))=v\left(x(P)-a_{2}\right)=0,
$$

which also contradicts (14).

Second case: $v\left(a_{1}\right)=1$. In this case the group $\operatorname{Im} \theta$ cannot be trivial, since it contains $\theta\left(P_{1}\right)=\left(1,{ }^{*}\right)$. We distinguish two cases.

(1) If $v\left(a_{2}\right) \geqslant 1$, then $\operatorname{Im} \theta=\mathbb{Z} / 2 \times \mathbb{Z} / 2$. Indeed, either $v\left(a_{2}\right)$ is even, in which case $\theta\left(P_{0}\right)=(1,1)$ and $\theta\left(P_{1}\right)=(1,0)$, or $v\left(a_{2}\right)$ is odd, in which case $\theta\left(P_{0}\right)=(0,1)$ and $\theta\left(P_{1}\right)=(1, *)$.

(2) If $v\left(a_{2}\right)=0$, then $\operatorname{Im} \theta$ is isomorphic to $\mathbb{Z} / 2$, with generator $(1,1)$. Indeed, this is the image of $P_{0}$ and $P_{1}$. Besides, for $P \in U(k)$, we have

$$
v(x(P)) \text { odd } \Rightarrow v\left(x(P)-a_{2}\right)=0 ;
$$

hence $v\left(x(P)-a_{1}\right)$ cannot be even, since this is incompatible with (14). Similarly,

$$
\begin{aligned}
v(x(P)) \text { even and } v\left(x(P)-a_{1}\right) \text { odd } & \Rightarrow v(x(P)) \geqslant 2 \\
& \Rightarrow v\left(x(P)-a_{2}\right)=0,
\end{aligned}
$$

which is incompatible with (14).

Proposition 4.8. Up to birational equivalence over $k$, the following classes of projective varieties coincide:

(i) cubic surfaces $V_{1} \subset \mathbb{P}_{k}^{3}$ with two conjugate double points, and no other singularities;

(ii) smooth cubic surfaces $V_{2} \subset \mathbb{P}_{k}^{3}$ with three coplanar $k$-rational lines and a further pair of coplanar conjugate lines;

(iii) smooth intersections of two quadrics $V_{3} \subset \mathbb{P}_{k}^{4}$ with a pair of conjugate lines meeting in a point;

(iv) intersections of two quadrics $V_{4} \subset \mathbb{P}_{k}^{4}$ with two conjugate double points and no other singularities, and such that $V_{4}(k) \neq \varnothing$;

(v) generalized Châtelet surfaces $V_{5} \subset \mathbb{P}_{k}^{1} \times \mathbb{P}_{k}^{2}$ with a $k$-point; in affine space $\operatorname{Spec} k[x] \otimes k[y, z], V_{5}$ is defined by an equation of the form

$$
N_{k(\delta) / k}(y+\delta z)=g(x) \text {, }
$$

where $g \in k[x]$ is a separable polynomial of degree 4 , and $[k(\delta): k]=2$. 
Proof. Let $V_{1}$ be a surface in Class (i). If $V_{1}$ is not of type $2 A_{1}$, it follows from Theorem 2.2 that $V_{1}$ is $k$-birationally equivalent to a cubic surface of type $2 A_{1}$. Hence, up to birational equivalence, (i) is the same as

(i) cubic surfaces $V_{1}^{\prime} \subset \mathbb{P}_{k}^{3}$ with two conjugate double points of type $2 A_{1}$, and no other singularities.

We show that Class (i), or (i)' ${ }^{\prime}$, coincides with all the others.

A surface in Class (i)' is $k$-birationally equivalent to one in Class (ii), by virtue of Theorem 2.2; conversely, one goes from (ii) to (i) by means of Proposition 4.1.

One goes from (i)' to (iii) by means of Corollary 2.3; and conversely, from (iii) to (i) by means of Proposition 4.5.

Let $V_{1}^{\prime}$ be a surface in Class (i)'. We consider the line denoted $L_{P^{*}}$ in Fig. 3: as explained in the proof of Lemma 1.2, it is the line residual to $L_{0}$ in the intersection of $V_{1}^{\prime}$ with its tangent plane at an arbitrary smooth point on the line $L_{0}$ joining the two double points. Its existence and basic properties can also be ascertained by a local calculation (starting from equation (2) of $\S 2$ ), which is only moderately tedious. As $L_{P^{*}}$ is an exceptional divisor of the first kind, defined over $k$, we can blow it down. In this way we get a singular Del Pezzo surface $V_{4}$ of degree 4 (cf. § 7) with the same singularities as $V_{1}^{\prime}$. By Example 0.7(a), $V_{4}$ can be realized as an intersection of two quadrics in $\mathbb{P}_{k}^{4}$. Clearly, $V_{4}(k) \neq \varnothing$; for instance, $V_{4}$ contains the image of $L_{P^{*}}$.

Conversely, let $V_{4}$ be a surface in Class (iv). We shall see in $\$ 7$ (Lemma 7.1) that $V_{4}$ is $k$-unirational. Thus we can find a smooth point $S \in V_{4}(k)$ which does not lie on any of the finitely many lines of $\bar{V}_{4}$ (cf. Lemma 5.3). (This is clear if $k$ is infinite. It is also true for $k$ finite with more than five elements, as one checks by going through the arguments of Lemmas 7.1 and 1.2.) By the argument of Lemma 5.3, one sees that the variety obtained by blowing up $S$ on $V_{4}$ embeds as a cubic surface in $\mathbb{P}_{k}^{3}$, which is in Class (i).

Finally, a surface in Class (i)' is $k$-birationally equivalent to one in Class (v), as we have already seen in the Remark following Proposition 4.1. (The case of characteristic 2 can be done with only minor changes.) Of course, $V_{5}(k) \neq \varnothing$, since $V_{1}^{\prime}(k) \neq \varnothing$. Conversely, on setting $u=x^{2}$ one sees that the generalized Châtelet surface with equation

$$
N_{k(\delta) / k}(y+\delta z)=g(x)=a_{0} x^{4}+\ldots+a_{4}
$$

is $k$-birationally equivalent to the intersection of two quadrics $V_{4}$ defined by

$$
\left\{\begin{array}{l}
x^{2}=u v, \\
N_{k(\delta) / k}(y+\delta z)=a_{0} u^{2}+a_{1} u x+a_{2} u v+a_{3} x v+a_{4} v^{2} .
\end{array}\right.
$$

An easy calculation, using the separability of $g$, shows that $V_{4}$ has precisely two conjugate double points, that is, $V_{4}$ is in Class (iv).

COROLlARY 4.9. Let $V$ be a smooth proper surface which is $k$-birationally equivalent to a surface belonging to any one of the Classes (i) to (v) of Proposition 4.8. In particular, $V(k) \neq \varnothing$. Then all the birational properties of generalized Châtelet surfaces hold for the surface $V$. We quote but a few, under the assumption that $\operatorname{char}(k)=0$.

(a) Let $\mathscr{T}$ be a universal torseur over $V$. If $\mathscr{T}$ has a $k$-point then $\mathscr{T}$ is stably $k$-rational. 
(b) If $k$ is a number field then the Hasse principle and weak approximation hold for $\mathscr{T}$.

(c) If $k$ is finitely generated over $\mathbb{Q}$ then $V(k) / R$ is finite, and each class for $R$-equivalence is parametrized by the $k$-points of a $k$-rational variety.

(d) Given $O \in V(k)$, the natural map $V(k) / R \rightarrow A_{0}(V)$, defined by $P \mapsto \operatorname{cl}(P-O)$, is a bijection.

(e) For $k$ a number field, the arithmetic conjecture discussed in [38], which enables one to compute $A_{0}(V)$, is verified.

(f) If $k$ is a number field then Brauer's obstruction is the only obstruction to weak approximation on $V$.

Proof. See [15, Chapter VIII, Theorems 1, 2, 6, 8, 10, and 11]. Some further properties can also be found there.

Remarks. (a) The proof of Proposition 4.8 shows that a surface $V_{5}$ in Class (v) is birationally equivalent to one in Class (iv) even if $V_{5}(k)=\varnothing$. Conversely, one can show that a surface $V_{4}$ in Class (iv), even without a $k$-point, is birationally equivalent to a generalized Châtelet surface, provided that some condition is fulfilled. See, for instance, Proposition 7.5.

(b) Consider the class

(i)" cubic surfaces $V_{1}^{\prime \prime} \subset \mathbb{P}_{k}^{3}$ with two conjugate double points of type $2 A_{2}$, and no other singularities.

Contrary to (i)', this class is strictly included in Class (i). Indeed,

Proposition 4.10. The surface $V_{3}$ of Example 3.3, which is in Class (i)', is not Q-birationally equivalent to a surface in Class (i)".

Proof. The conic bundle surface $V_{1}$ of Example 3.3 is of Type VIII in Manin's classification [32, Chapter IV, §9]. This is proved in [29, Propositions 1.12 and 1.10]. Now it follows from [32, loc. cit., Table 3], that $H^{1}(\mathbb{Q}$, Pic $\bar{U})=\mathbb{Z} / 2$, where $U$ is any smooth proper model of either $V_{1}$ or $V_{3}$, for instance, the Del Pezzo surface defined by (4) in Example 3.3.

But it can be shown, with the methods of Part II, that any surface in (i)" is $k$-birationally equivalent to a smooth Del Pezzo surface $Z$ of degree 4 , whose diagram of lines contains a connected pair of orbits of order 2:

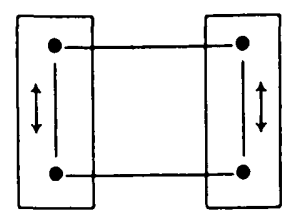

Indeed, starting from the diagram of irreducible roots and lines given at the end of $\S 1$ for the case $2 A_{2}$, we blow down the line $l_{14}$. This gives rise to a generalized Del Pezzo surface of degree 4, of Type 3 in the classification of $\S 6$. There is an 
orbit of order 2 coming from the pair of roots $\left\{l_{1}-l_{2}, l_{4}-l_{5}\right\}$ :

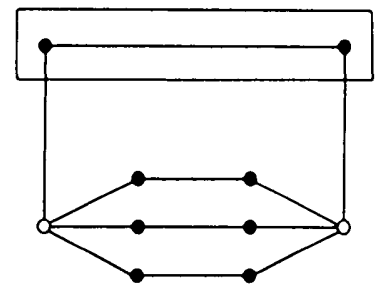

Now if we blow up a general $\mathbb{Q}$-point on this surface, we obtain a generalized Del Pezzo surface $V^{\prime}$ of degree 3 and of type $2 A_{1}$. By Proposition 0.6, this is a model of a singular cubic surface of type $2 A_{1}$. The line $l$ joining the two double points corresponds, on $V^{\prime}$, to a subdiagram of the form

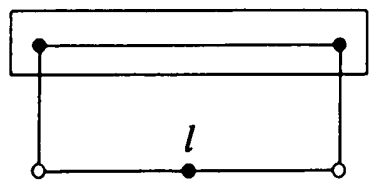

Blowing down the line $l$ on $V^{\prime}$, we obtain a subdiagram of the form

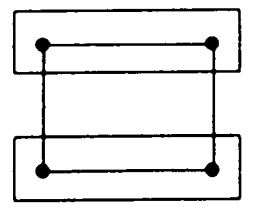

as required.

With this information on the orbits, a glance at Table 2 of [32, Chapter IV, $\S 9]$, shows that the smooth Del Pezzo surface $Z$ must belong to one of the following types: II, IV, or VII (it is now known (cf. [27]) that Type V does not exist, but has to be replaced by another type, which does not contain any orbit of order 2). But if we suppose that $Z$ is a model of either $V_{1}$ or $V_{3}$, we must have

$$
H^{1}(\mathbb{Q}, \operatorname{Pic} \bar{Z})=H^{1}(\mathbb{Q}, \operatorname{Pic} \bar{U})=\mathbb{Z} / 2 .
$$

Then $Z$ must be of Type VII, as follows from Table 3 of [32, loc. cit.].

This yields a contradiction. Indeed, by Theorem 1 of [27], we know that the full group of automorphisms of a surface of Type VII is $(\mathbb{Z} / 2)^{2}$. This must coincide with the splitting group of the surface, since there is also an orbit of order 4. Hence, by [29, Proposition 1.10 and Theorem 1.9], the Néron-Severi torus $S$ of $Z$ is rational. But we know that this is not the case for the surfaces of Example 3.3, for which $H^{1}(\mathbb{Q}, S) \neq 0$. (Cf. Property (c) in the Remark following the example.) 


\section{PART II. INTERSECTIONS OF TWO QUADRICS}

\section{Classification of singularities}

The singular Del Pezzo surfaces of degree at least 4 have been studied classically. Timms [44], in particular, gives an impressive list of possibilities, which includes in each case a description of the lines on the surface. As mentioned in the Introduction, a nearly modern presentation was given by $\mathrm{Du}$ Val [20]. However, a proof that the singularities of the non-conical intersection of two quadrics in $\mathbb{P}_{\mathbb{C}}^{4}$ are always rational was obtained only recently $[37,26,47]$. The present section is meant to provide number theorists with the minimum background for understanding the forthcoming sections.

To begin with, we show how the rationality statement of Proposition 0.3(b) follows from Proposition 0.2. The reason for giving this argument is that it depends on two lemmas which are referred to elsewhere in the paper.

Proposition 5.1. Let $V \subset \mathbb{P}_{\mathbb{C}}^{4}$ be an irreducible intersection of two quadrics which has only isolated singularities. Then either $V$ is a cone over a smooth quartic of genus 1 in $\mathbb{P}_{\mathbb{C}}^{3}$, or $V$ has only rational double points.

Proof. If $V$ is a cone, then a general hyperplane section of $V$ is the intersection of two quadrics in $\mathbb{P}_{\mathbb{C}}^{3}$. This intersection is necessarily smooth, since $V$ has only isolated singularities. Thus we may assume that $V$ is not a cone. Take a general point $S$ on $V$, and project the surface $V$ from $S$ into $\mathbb{P}_{\mathbb{C}}^{3}$. Call this projection $\varphi$, and let $W=\varphi[V]$ be its image.

Lemma 5.2. The surface $W \subset \mathbb{P}_{\mathbb{C}}^{3}$ is a cubic surface with only isolated rational double points.

Proof. By the argument of Proposition 4.5, we see that the projection $\varphi: V \cdots \rightarrow W$ is generically one-to-one. Hence the degree of $W$ is equal to 3 (the intersection of $W$ with a general line in $\mathbb{P}_{C}^{3}$ corresponds to the intersection of $V$ with a general 2-plane through $S$ ). Next we consider a general hyperplane section $\Gamma$ of $V$ through $S$. Since $S$ is a general point of $V$, which has only isolated singularities, we see that $\Gamma$ has genus 1 . Therefore its image, which is a general plane section of $W$, is also a curve of genus 1 . This implies that $W$ has only isolated singularities.

Moreover, $W$ is not a cone over an elliptic curve, since it is a rational variety. Indeed $W$ is birationally equivalent to $V$, which is not a cone. Hence either $V$ is smooth, in which case it is well known to be rational, or it has a multiple point $Q$, which is not a vertex. Projecting from $Q$, we get a surface of degree at most 2 , which is birationally equivalent to $V$. It follows that $V$ (and hence $W$ ) is rational. Thus we are in Case (b) of Proposition 0.2, which proves the lemma.

Let $\tilde{V}$ be the variety obtained by blowing up $S$ on $V$. Consider the following commutative diagram:

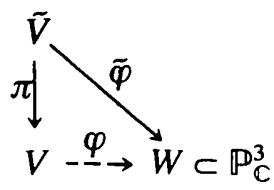


LEMMA 5.3. The intersection $V$ contains only finitely many lines. Hence $\tilde{\varphi}$ is an isomorphism.

Proof. If $V$ contained infinitely many lines, then so would $W$. But, by Lemma $5.2, W$ is neither a cone, nor a cubic surface with a double line. Hence $W$ contains only finitely many lines, and this proves the first assertion.

To show that $\tilde{\varphi}$ is an isomorphism, one can proceed as in Lemma 2 of [17]. Using the fact that $V$ is the intersection of two quadrics, one shows that the linear system $\mathscr{L}$ of all hyperplanes through $S$ separates points and infinitely near points on $V \backslash\{S\}$. Indeed $S$, being a general point of $V$, does not lie on any line of $V$, since there are only finitely many of them. Then one shows that $\mathscr{L}$ separates the points of $L=\pi^{-1}(S)$, that is, the points infinitely close to $S$. It follows that $\tilde{\varphi}$ is a morphism with no infinite fibres. Now, by Lemma 5.2, $W$ is normal. Hence, by the Zariski Main Theorem, $\tilde{\varphi}$ is an isomorphism.

On combining Lemmas 5.2 and 5.3, one sees that $V$ has only rational double points, which completes the proof of Proposition 5.1.

This proposition enables us to use the results of Demazure [19] on generalized Del Pezzo surfaces. In the rest of this section, $k$ denotes an arbitrary algebraically closed field.

Lemma 5.4. Suppose $V \subset \mathbb{P}_{k}^{4}$ has only rational double points. Let $f: V^{\prime} \rightarrow V$ be the minimal desingularization of $V$. Then Pic $V^{\prime}$ is a free abelian group of rank 6 , and the triple $\left(\mathrm{Pic} V^{\prime} ; \omega_{V^{\prime}} ;{ }^{\prime}\right)$, where $\omega_{V^{\prime}}$ denotes the canonical class and ${ }^{\prime} V^{\prime}$ the intersection product, is isomorphic to the corresponding triple for a smooth Del Pezzo surface of degree 4.

Proof. By Example 0.7(b), $V^{\prime}$ is a generalized Del Pezzo surface of degree 4. Moreover, Pic $V^{\prime}$ is free of rank $10-\left(\omega_{V^{\prime}}^{2}\right)=6$ (cf. [32, Chapter IV, Lemma 2.4]). The final assertion follows from Proposition 0.4 and the construction of the surfaces $\mathbb{P}^{2}(\Sigma)$ in $[19$, Part II, $\S 2]$.

The following facts about the triple (Pic $V^{\prime} ; \omega_{V^{\prime}} ; \cdot v^{\prime}$ ) are taken from [19]: Pic $V^{\prime} \simeq \mathbb{Z}^{6}$ has a basis $l_{0}, l_{1}, \ldots, l_{5}$ such that

$$
\left(l_{0}^{2}\right)=1, \quad\left(l_{i}^{2}\right)=-1 \text { for all } i>0, \quad\left(l_{i} \cdot l_{j}\right)=0 \quad \text { for all } i \neq j .
$$

Let $Q=\left\{\alpha \in \operatorname{Pic} V^{\prime} \mid\left(\alpha . \omega_{V^{\prime}}\right)=0\right\}$ be the orthogonal space to

$$
\omega_{V^{\prime}}=-3 l_{0}+\sum_{h=1}^{5} l_{h}
$$

Let $R=\left\{\alpha \in Q \mid\left(\alpha^{2}\right)=-2\right\}$ be the set of roots. Then $R$ consists of all elements of the form $l_{i}-l_{j}$ or $\pm\left(l_{0}-l_{i}-l_{j}-l_{k}\right)$, with $i, j, k$ all distinct and non-zero. We call simple roots the elements $l_{0}-l_{1}-l_{2}-l_{3}, l_{1}-l_{2}, l_{2}-l_{3}, l_{3}-l_{4}$, and $l_{4}-l_{5}$. They form a basis of the $\mathbb{Z}$-module $Q$, with associated Dynkin diagram $D_{5}$. The extended Dynkin diagram $\tilde{D}_{5}$ is obtained by considering the root $-l_{0}+l_{3}+l_{4}+l_{5}$ 
in addition to the simple roots:

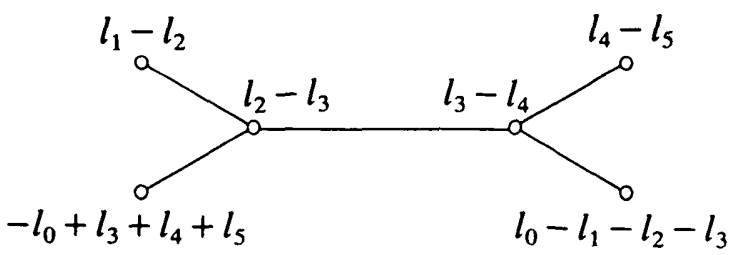

FIG. 4

Remark. It is clear from Proposition 0.1 that the set of irreducible curves $E_{i} \subset V^{\prime}$ lying above the singular points of $V$ can be identified with a certain subset $R_{i}$ of $R$. This subset consists precisely of all the roots $\alpha$ which are represented by an irreducible curve $\Gamma$ on $V^{\prime}$. Indeed the relations $\left(\alpha^{2}\right)=-2$ and $\left(\alpha . \omega_{V^{\prime}}\right)=0$ imply that $p_{a}(\Gamma)=0$, and hence $\Gamma$ is a smooth rational curve. Moreover, $\Gamma$ is uniquely determined, since $\left(\alpha^{2}\right)<0$. The elements of $R_{i}$ will be called irreducible roots, as in the Introduction.

Proposition 5.5 (Demazure). The subset $R_{i}$ is a free subset of Pic $V^{\prime}$. A necessary and sufficient condition for an exceptional class $\lambda \in$ Pic $V^{\prime}$ (that is, one satisfying $\left.\left(\lambda^{2}\right)=\left(\lambda . \omega_{V^{\prime}}\right)=-1\right)$ to be the class of an irreducible effective divisor $L$, is that $(\lambda . \alpha) \geqslant 0$ for all $\alpha \in R_{i}$.

This is proved in [19, Theorem III.2 and Corollary]. Clearly, $L$ is uniquely determined. Since $\omega_{V^{\prime}}=f^{*}\left(\omega_{V}\right)$, we see that $L$ is nothing but the proper transform of a straight line on $V$.

We end this section by enunciating the stronger form of Proposition 0.3(b), which classifies all intersections of two quadrics in $\mathbb{P}_{k}^{4}$ having only isolated rational double points. This classification is somewhat more precise than the classification into types, since there are two different kinds of varieties with singularities of type $2 A_{1}$, and two of type $A_{3}$. In every case of Proposition 5.6, the representative set $R_{i}$ appears as a proper subgraph of the Dynkin diagram $\tilde{D}_{5}$ of Fig. 4. (Of course, two vertices which are joined by an edge in $\tilde{D}_{5}$, must also be joined by an edge in any subgraph.) But the signs have been selected so that the vertex $\alpha=$ $-l_{0}+l_{3}+l_{4}+l_{5}$ is replaced by its opposite. Indeed $-\alpha$ has a standard realization: take a line $L$ in $\mathbb{P}_{k}^{2}$, and blow up three distinct points $x_{3}, x_{4}, x_{5}$ lying on it; then $-\alpha$ is the class of the proper transform of $L$.

It is worth remarking that there does not seem to be any obvious fundamental reason why the type of singularity should be a proper subgraph of $\widetilde{D}_{5}$. Indeed, Naruki and Urabe [36] have given examples of singular Del Pezzo surfaces of degree 2 whose type is not a proper subgraph of the relevant Dynkin diagram $\widetilde{E}_{7}$. (For instance, one can produce $6 A_{1}$ by blowing up the seven vertices of the plane figure shown in Fig. 5 and then contracting the six irreducible roots thus obtained.)

Proposition 5.6 (Du Val). Let $V \subset \mathbb{P}_{k}^{4}$ be a singular intersection of two quadrics which has only isolated rational double points. Let $R_{i} \subset R$ be the set of irreducible roots of its minimal desingularization $V^{\prime}$. Then, up to the action of the Weyl group $W(R)$, the set $R_{i}$ belongs to one of the following types. 


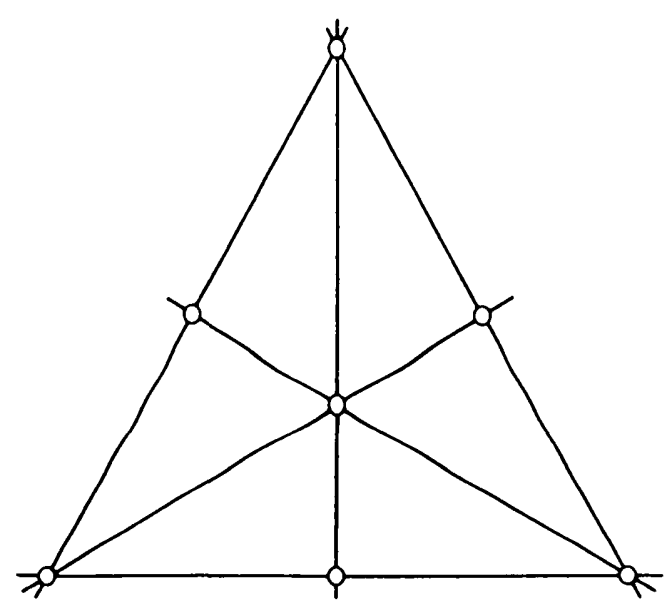

FIG. 5

1. $l_{4}-l_{5}$.

2. $l_{2}-l_{3}, l_{4}-l_{5}$.

(The line joining the two double points lies on $V$. )

3. $l_{0}-l_{1}-l_{2}-l_{3}, l_{4}-l_{5}$.

(The line joining the two double points does not lie on $V$.)

4. $l_{3}-l_{4}, l_{4}-l_{5}$.

5. $l_{2}-l_{3}, l_{0}-l_{1}-l_{2}-l_{3}, l_{4}-l_{5}$.

6. $l_{1}-l_{2}, l_{3}-l_{4}, l_{4}-l_{5}$.

7. $l_{2}-l_{3}, l_{3}-l_{4}, l_{4}-l_{5}$.

( $V$ contains five lines, only three of which pass through the double point.)

8. $l_{0}-l_{1}-l_{2}-l_{3}, l_{3}-l_{4}, l_{4}-l_{5}$.

( $V$ contains four lines, all of which pass through the double point.)

9. $l_{1}-l_{2}, l_{0}-l_{3}-l_{4}-l_{5}, l_{0}-l_{1}-l_{2}-l_{3}, l_{4}-l_{5}$.

10. $l_{1}-l_{2}, l_{2}-l_{3}, l_{0}-l_{1}-l_{2}-l_{3}, l_{4}-l_{5}$.

11. $l_{1}-l_{2}, l_{0}-l_{1}-l_{2}-l_{3}, l_{3}-l_{4}, l_{4}-l_{5}$

12. $l_{1}-l_{2}, l_{2}-l_{3}, l_{3}-l_{4}, l_{4}-l_{5}$.

13. $l_{2}-l_{3}, l_{3}-l_{4}, l_{0}-l_{1}-l_{2}-l_{3}, l_{4}-l_{5}$.

14. $l_{1}-l_{2}, l_{0}-l_{3}-l_{4}-l_{5}, l_{0}-l_{1}-l_{2}-l_{3}, l_{3}-l_{4}, l_{4}-l_{5}$.

15. $l_{1}-l_{2}, l_{2}-l_{3}, l_{3}-l_{4}, l_{0}-l_{1}-l_{2}-l_{3}, l_{4}-l_{5}$.

Moreover, all these cases occur; that is, for each one of the sets $R_{i}$ listed above, there exists an intersection of two quadrics $V \subset \mathbb{P}_{k}^{4}$ whose set of irreducible roots in Pic $V^{\prime}$ is the given set $R_{i}$.

Sketch of proof (see also [47, Table 1]). The realizability of all these types follows from the construction of the generalized Del Pezzo surfaces of higher degree (cf. Proposition 8.6 and Example 0.7(a)).

The classification of all possibilities is based on the fact that $R$ is a root system of type $D_{5}$, for which a standard realization is known [5, Chapter VI, $\S 4$, no. 8, 
and Table IV at the end of the volume]. However, to avoid confusion when referring to Proposition 5.5, we shall work with the intersection product on Pic $V^{\prime}$, which is negative definite on $Q$ (the orthogonal space to $\left.\omega_{V^{\prime}}\right)$. Thus $(\alpha . \beta)$ is the opposite of the standard inner product $(\alpha \mid \beta)$ appearing in Bourbaki.

Let $\left\{\varepsilon_{1}, \ldots, \varepsilon_{5}\right\}$ denote the canonical basis of $\mathbb{R}^{5}$, with intersection matrix $-I$. We know from [5, loc. cit.] that $R=\left\{ \pm \varepsilon_{i} \pm \varepsilon_{j} \mid 1 \leqslant i<j \leqslant 5\right\}$. We can identify these roots with elements of Pic $V^{\prime}$ through the following correspondence:

$$
\begin{aligned}
l_{i}-l_{j} & \leftrightarrow \varepsilon_{i}-\varepsilon_{j}, \\
l_{0}+l_{i}+l_{j}-\sum_{h=1}^{5} l_{h} & \leftrightarrow \varepsilon_{i}+\varepsilon_{j} .
\end{aligned}
$$

This amounts to setting

$$
\varepsilon_{i}=l_{i}+\frac{1}{2}\left(l_{0}-\sum_{h=1}^{5} l_{h}\right)
$$

and it provides an interesting realization of the root system $D_{5}$ in the space $\mathbb{R}^{6}$ spanned by the $l_{i}$, with the associated indefinite bilinear form. Further, the Weyl group $W(R)$ is known to be the semi-direct product of the symmetric group $\mathscr{S}_{5}$ (which acts by permuting the base vectors $\varepsilon_{i}$ ) with the group $(\mathbb{Z} / 2)^{4}$ consisting of all even combinations of sign changes $c_{i}: \varepsilon_{i} \mapsto-\varepsilon_{i}$.

Now it follows from Proposition 5.5 and an obvious geometric constraint that it suffices to classify the subsets $R_{i} \subset R$ with the following two properties:

(a) $R_{i}$ consists of linearly independent vectors (whence we already see that $R_{i}$ contains at most five elements);

(b) for any two distinct roots $\alpha, \beta \in R_{i}$, we have $(\alpha . \beta)=0$ or +1 .

This is done by induction on the cardinality of $R_{i}$.

\section{Diagrams of irreducible roots and lines}

With the help of Proposition 5.5 we can give a complete description of the lines that lie on a singular intersection of two quadrics in $\mathbb{P}_{k}^{4}$. We recall that the exceptional classes in Pic $V^{\prime}$ are the following:

$$
\begin{gathered}
l_{i} \quad \text { for } i \geqslant 1 ; \\
l_{i j}=l_{0}-l_{i}-l_{j} \quad \text { for } 1 \leqslant i<j ; \\
q=2 l_{0}-\sum_{h=1}^{5} l_{h} .
\end{gathered}
$$

As was already mentioned after Proposition 5.5, those exceptional classes which are represented by an irreducible effective divisor $L$ on $V^{\prime}$, correspond to the straight lines of $V$. By an abuse of language (already used in the Introduction), we shall also call them the lines of $V^{\prime}$.

We can represent on the same diagram both the lines and the irreducible roots of $V^{\prime}$, by using two kinds of vertices: the irreducible roots are represented by 
small circles, while the lines are represented by dots. Two vertices are joined by an edge if and only if the corresponding divisors meet (in which case the intersection number is always equal to 1 ). Then Proposition 5.6 can be restated as Proposition 6.1 below.

Though the diagrams of Proposition 6.1 are drawn over an algebraically closed field, it is important to notice that they contain a wealth of arithmetic information. Indeed, for an arbitrary perfect field $k$, they give very precise information on the Galois action (the action of $\mathrm{Gal}(\bar{k} / k)$ on Pic $\left.\bar{V}^{\prime}\right)$. An example: if $V$ has exactly three double points, can the action of $\operatorname{Gal}(\bar{k} / k)$ be transitive on these points? A glance at Diagram 5 shows this to be impossible. We shall use these diagrams in a very essential way in our proof of Theorem 7.2.

Proposition 6.1. The configuration of irreducible roots and lines on the minimal desingularization of a singular Del Pezzo surface of degree 4 is described by one of the Diagrams 1-15.

1. (12 lines)

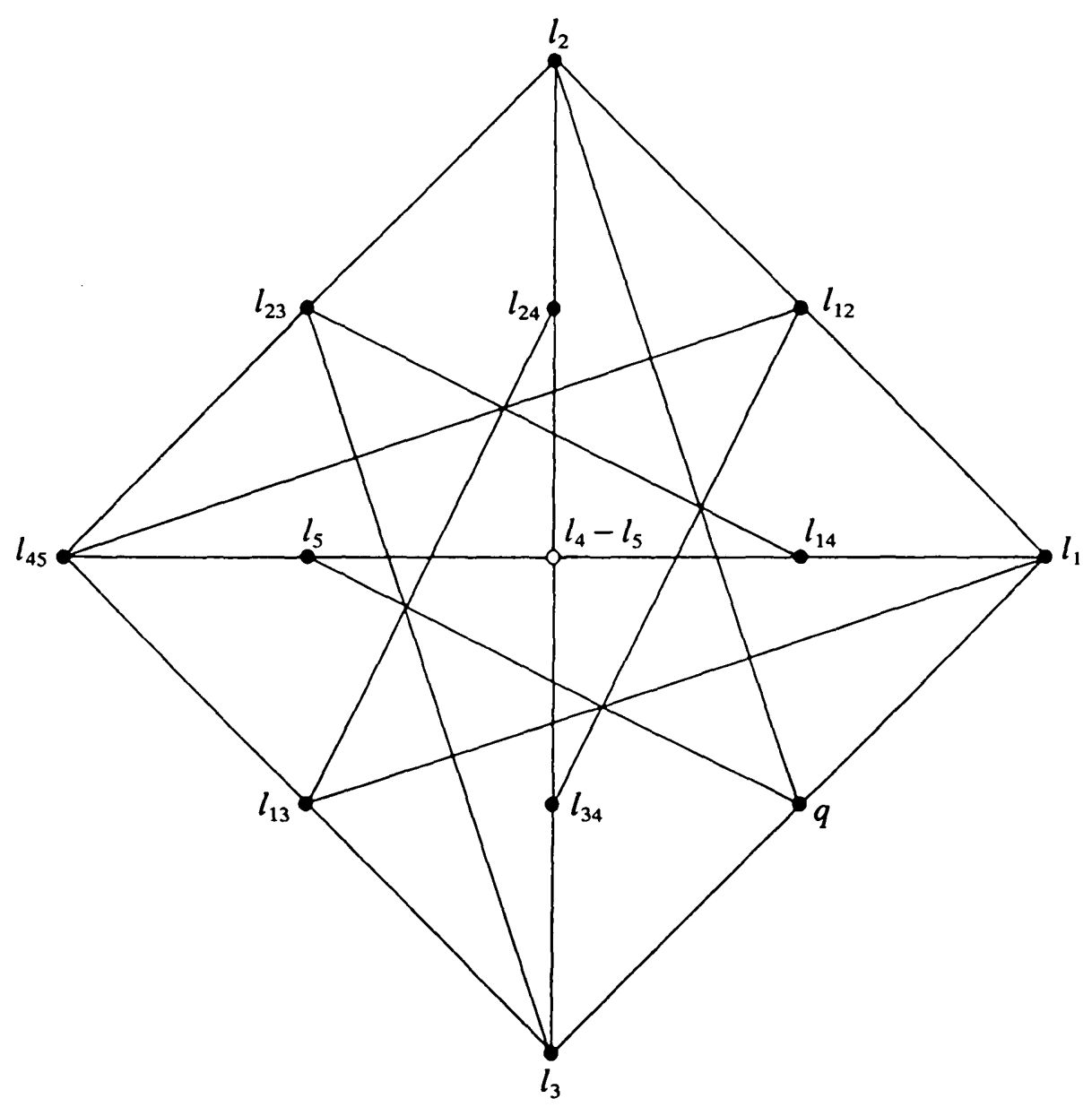


2. (9 lines)

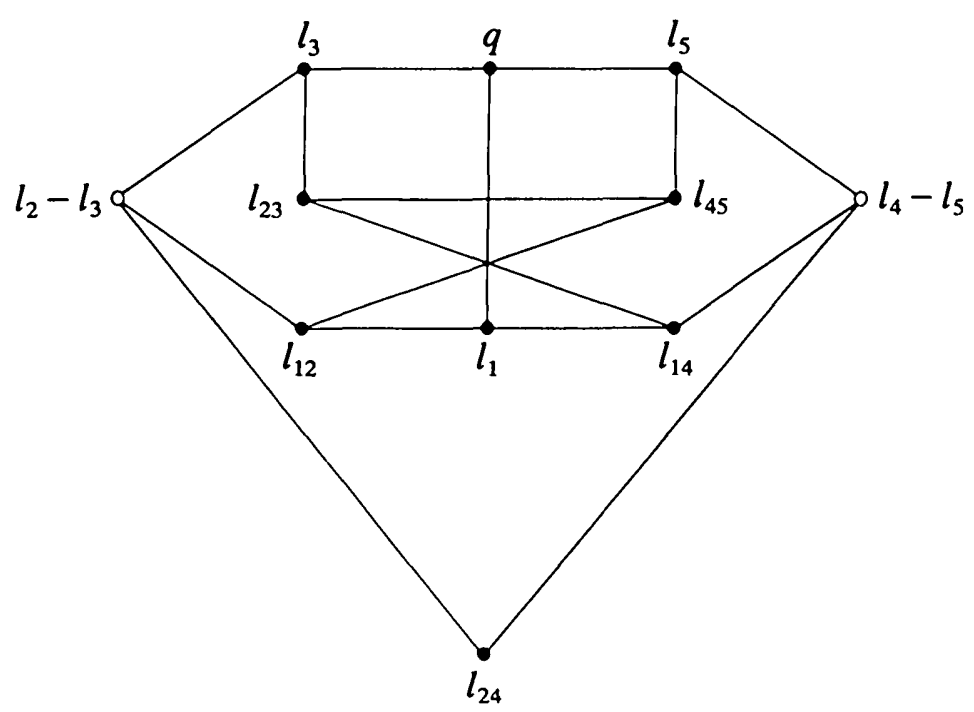

3. (8 lines)

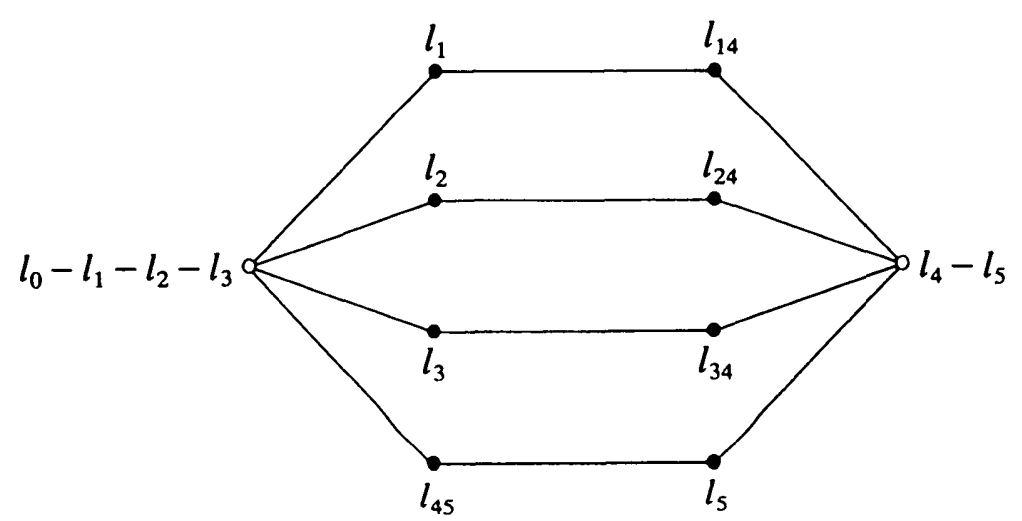

4. (8 lines)

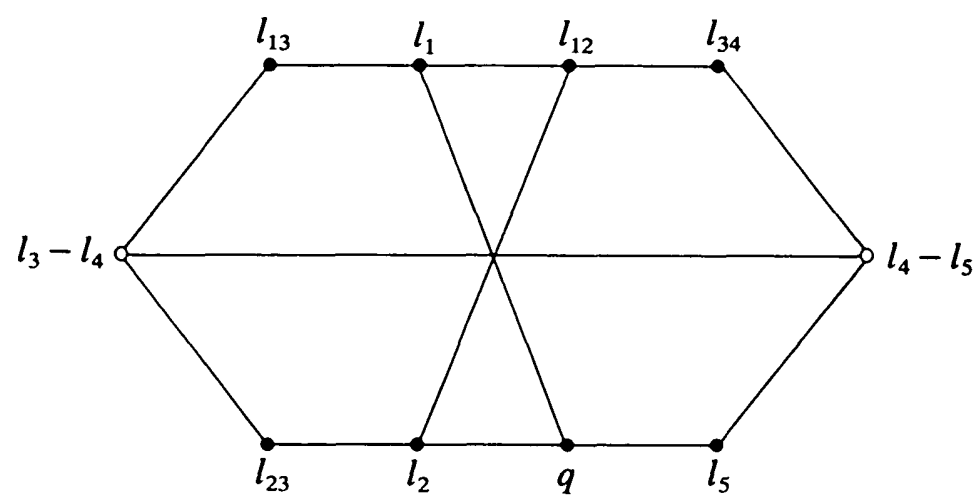


5. (6 lines)

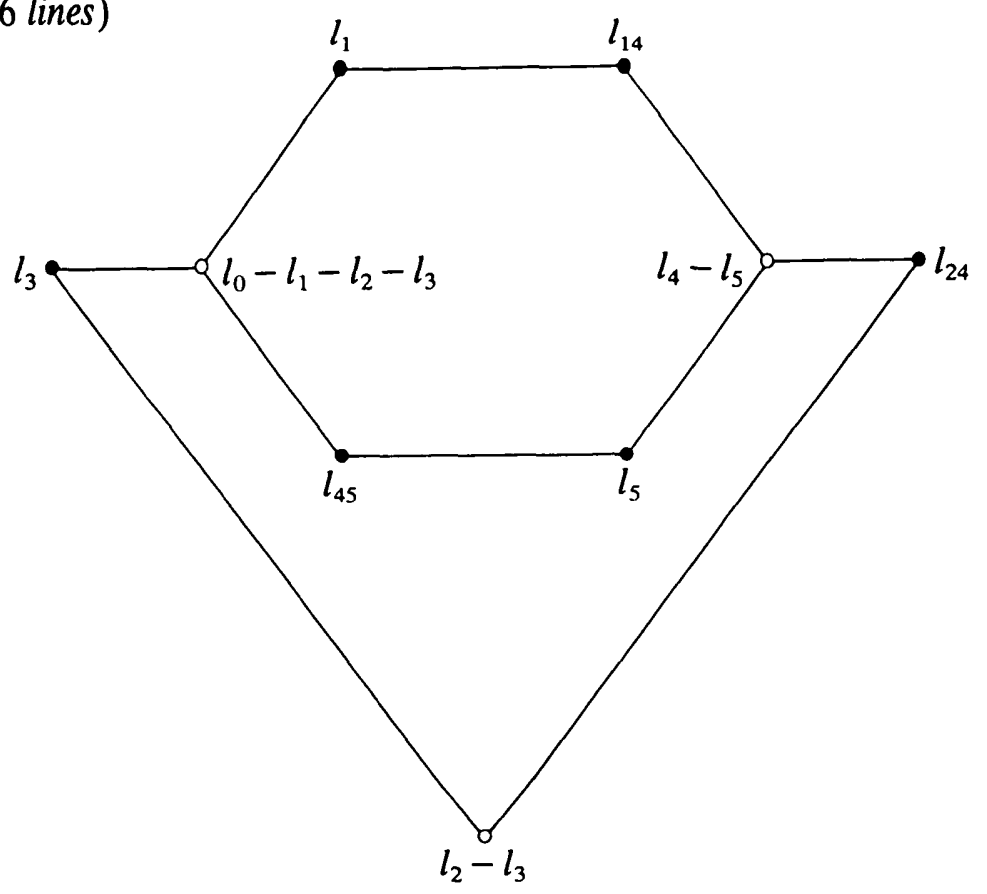

[3 $\left.A_{1}\right]$

6. (6 lines)

[ $\left.A_{1} A_{2}\right]$

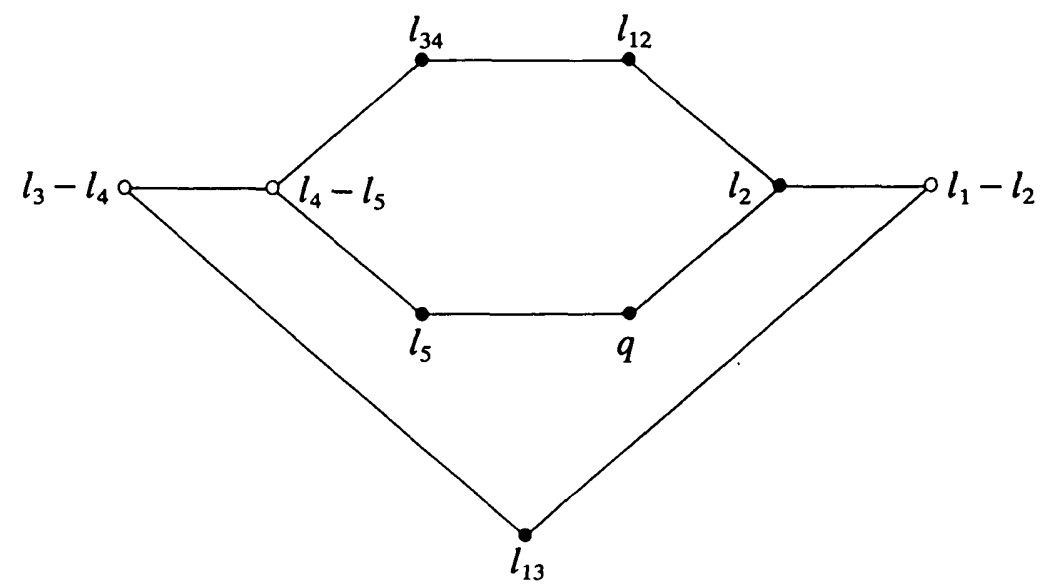

7. (5 lines)

[ $\left.A_{3}\right]$

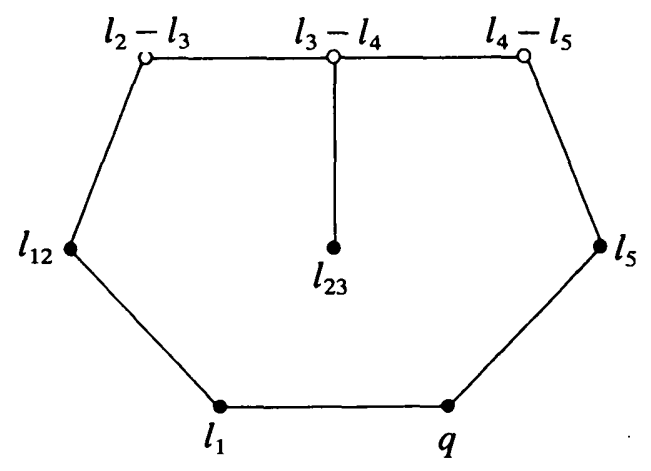


8. (4 lines)

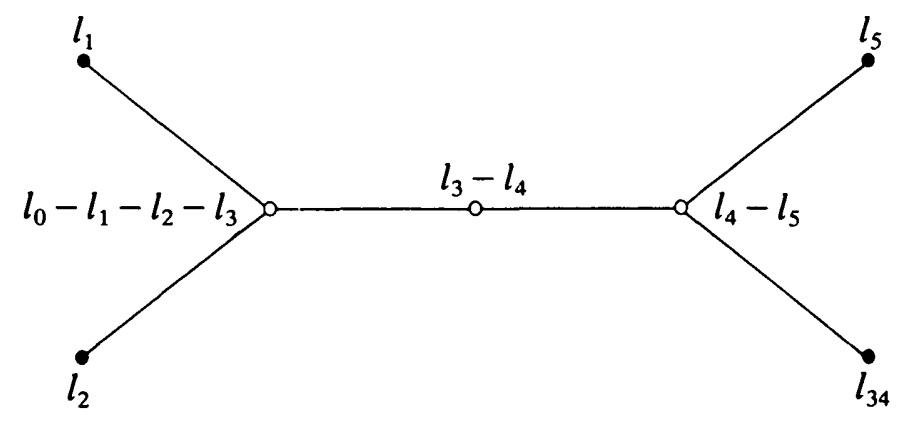

9. (4 lines)

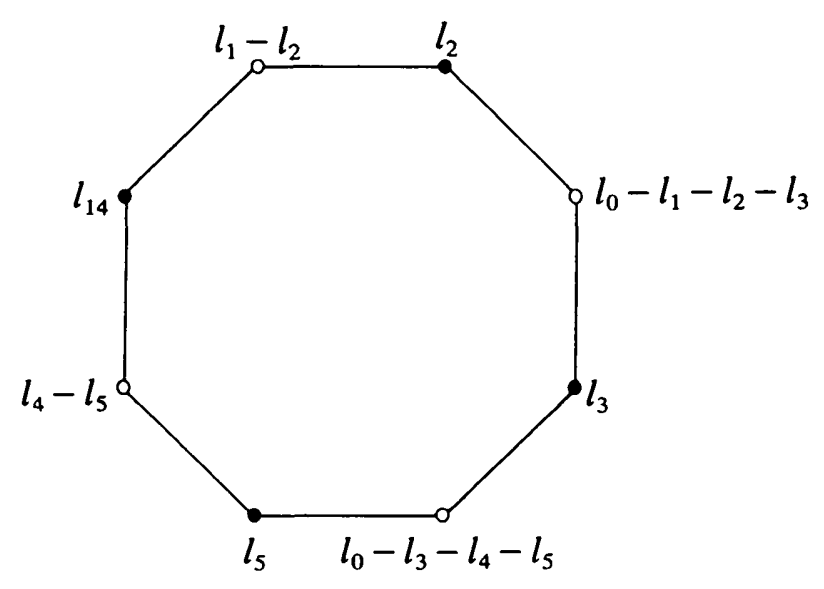

10. (4 lines)

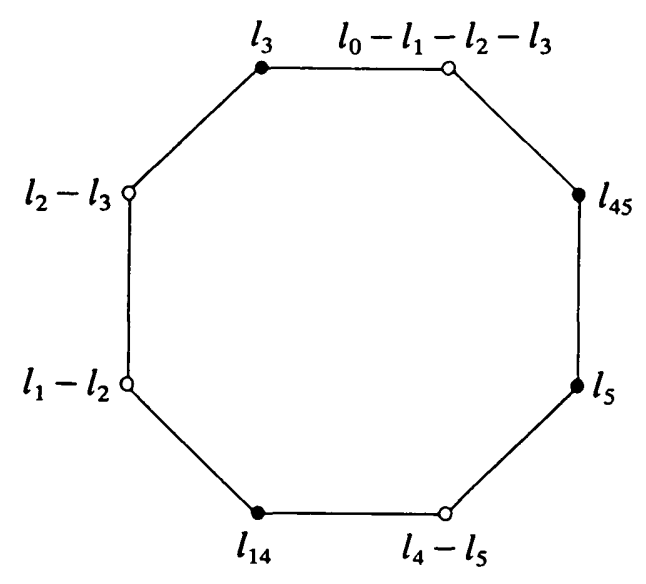


11. (3 lines)

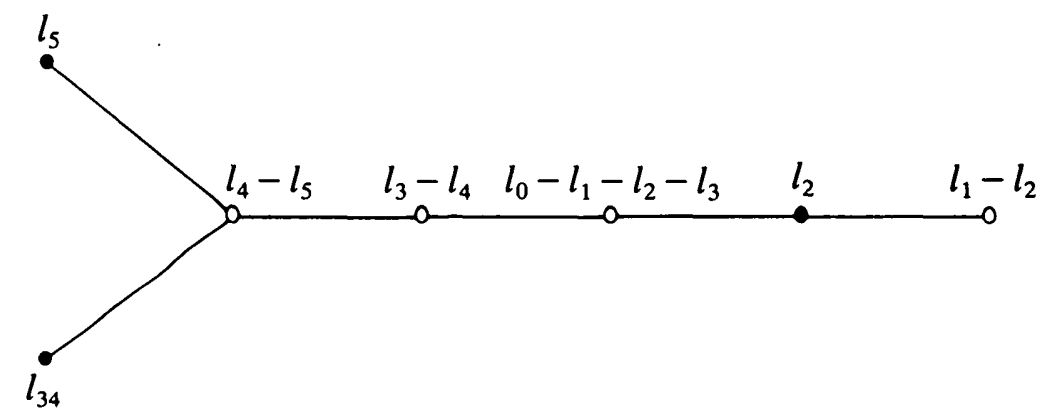

12. (3 lines)

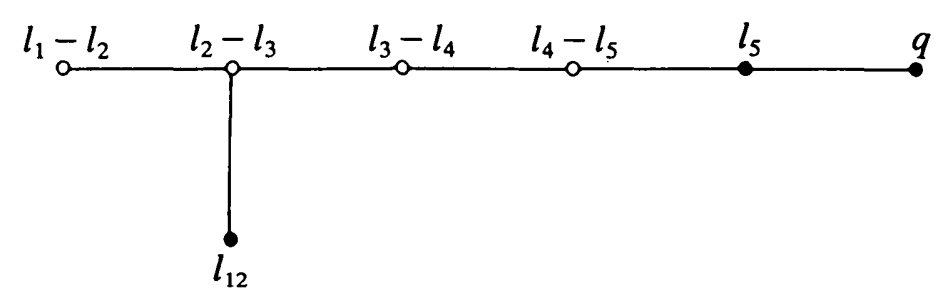

13. (2 lines)

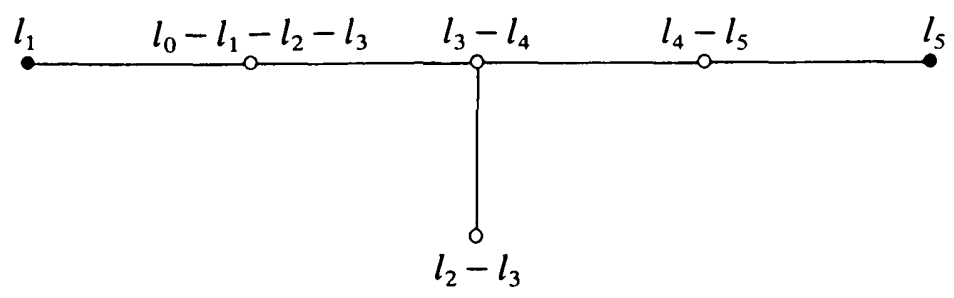

14. (2 lines)

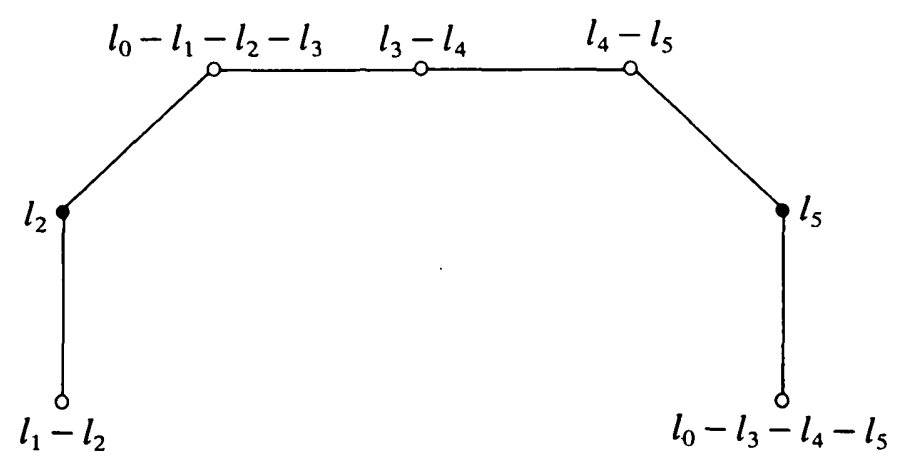


15. (1 line)

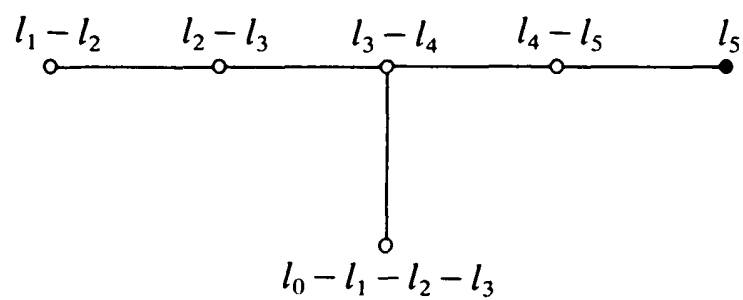

\section{Arithmetical applications}

In this section, $k$ will again denote an arbitrary perfect field. The intersections of two quadrics we consider will always be assumed irreducible, even when this is not mentioned explicitly. Among all singular intersections of two quadrics in $\mathbb{P}_{k}^{4}$, those belonging to the third type of Proposition 5.6 have special arithmetic properties:

Definition. We say that a singular intersection of two quadrics $V \subset \mathbb{P}_{k}^{4}$ is an Iskovskih surface if it has precisely two conjugate double points, with the property that the line joining them does not lie on $V$.

Proposition 5.6 shows that, in this case, both double points are of type $A_{1}$. Iskovskih [24] used a surface of this type to give the first example of an intersection of two quadrics in $\mathbb{P}_{Q}^{4}$ having smooth points everywhere locally, but no rational point. In Theorem 7.2 , we shall see that these are the only singular intersections of two quadrics in $\mathbb{P}_{k}^{4}$ for which the Clean Hasse Principle can fail.

Lemma 7.1. Let $V \subset \mathbb{P}_{k}^{4}$ be a singular intersection of two quadrics which has only isolated singularities and is not a cone. Suppose $V$ contains a smooth $k$-point. Then $V$ is $k$-unirational. More precisely, there is a dominant rational map $\mathbb{P}_{k}^{2} \cdots V$ of degree 1 or 2.

Proof. Let $S \in V(k)$ be a smooth $k$-point, and consider the projection $\varphi: V \ldots \mathbb{P}_{k}^{3}$ from $S$. By the argument of Proposition 4.5, the image $W=\varphi[V]$ is a cubic surface and $\varphi: V \cdots W$ is a birational equivalence. As we saw in the proof of Lemma 5.2,W is not a cone over an elliptic curve, since $\bar{V}$ (and hence $\bar{W}$ ) is $\bar{k}$-rational. Moreover, it follows from Nishimura's lemma (Lemma 1.0) that $W(k) \neq \varnothing$. This implies that $W$ (and hence $V$ ) is $k$-unirational. Indeed, as in the proof of Proposition 3.2(a), we may assume that the singularities of $W$ are isolated. Then the assertion follows from Lemmas 1.1 and 1.2. More precisely, $W$ is $k$-rational unless it has two conjugate double points, and no other singularities. But, in this case, Theorem 1.3(b) asserts that $W$ is birationally equivalent to a smooth Del Pezzo surface of degree 4 with a $k$-point. Now, such a surface is known to be $k$-unirational of degree 2 [32, Chapter IV, Theorem 7.8].

Remarks. (a) An easy refinement of this proof shows in fact that $V$ is $k$-rational if it is not an Iskovskih surface. We do not give the details, since Lemma 7.4 contains a more precise statement. 
(b) As we saw in $\S 3$, the Iskovskih surface $V_{2}$ of Example 3.3 contains a smooth $\mathbb{Q}$-point, but is not $\mathbb{Q}$-rational.

(c) Lemma 7.1 does not hold under the sole assumption that $V(k) \neq \varnothing$. Indeed one can give examples where all $k$-points on $V$ are singular, in which case $V$ is clearly not $k$-unirational. For instance, over $k=\mathbb{R}$ or $\mathbb{Q}_{2}$, we can take the variety $V$ defined by

$$
\left\{\begin{array}{l}
x_{0}^{2}-17 x_{1}^{2}=x_{2}^{2} \\
x_{0}^{2}-17 x_{1}^{2}=-x_{3}^{2}-x_{4}^{2}
\end{array}\right.
$$

This is 'Ellison's example' (cf. [12, Example 3.1.3]).

THEOREM 7.2. Let $\mathcal{V}$ be the family of all singular intersections of two quadrics $V \subset \mathbb{P}_{k}^{4}$ having only isolated rational double points. Let $\mathscr{V}_{0} \subset \mathscr{V}$ be the subfamily consisting of those varieties which are not Iskovskih surfaces.

(a) The minimal desingularization $V^{\prime}$ of any variety $V \in \mathscr{V}_{0}$ is a non-minimal surface (that is, $V^{\prime}$ contains an exceptional curve; cf. [32, Chapter III, §4.7]).

(b) If $k$ is a number field, then the 'Clean Hasse Principle' (called 'principe de Hasse fin' in [12]) holds for the class $\mathscr{V}_{0}$ : if $V \in \mathscr{V}_{0}$ contains a smooth point defined over $k_{v}$, for every completion $k_{v}$ of $k$, then every proper model of $V$ contains a $k$-point. (However, the usual Hasse principle may fail to hold; a counter-example is the system (1) over $k=\mathbb{Q}$.)

If $V \in \mathscr{V}_{0}$, let $Z$ be any smooth projective model of $V$.

(c) If $Z(k) \neq \varnothing$ then $Z$ is $k$-rational.

(d) The $\operatorname{Gal}(\bar{k} / k)$-module $\operatorname{Pic} \bar{Z}$ is stably a permutation module.

(e) The Néron-Severi torus of $Z$ is stably rational.

(f) For $k$ a local or global field of characteristic 0 , the Chow group $A_{0}(Z)$ is trivial.

(g) $R$-equivalence is trivial on $Z$, that is, $Z(k) / R=0$, provided $Z(k) \neq \varnothing$.

None of these assertions holds for the class $\mathscr{V} \backslash \mathscr{V}_{0}$ of Iskovskih surfaces.

Proof. Using the diagrams of $\S 6$, we shall establish a more precise version of (a), from which all the assertions derive. We begin by making some general remarks about the effect of blowing down a line $L$ on a generalized Del Pezzo surface $X_{1}$, defined over $\bar{k}$. This gives rise to a morphism $g: X_{1} \rightarrow X_{2}$, where $X_{2}$ is again a generalized Del Pezzo surface. (This is an easy consequence of the definition given in the Introduction.) In addition:

(i) $K_{X_{1}}=g^{*}\left(K_{X_{2}}\right)+L$, whence $\left(K_{X_{2}}^{2}\right)=\left(K_{X_{1}}^{2}\right)+1$;

(ii) if a curve $\Gamma_{1}$ meets $L$ with multiplicity $\mu=1$, then its image $\Gamma_{2}=g\left[\Gamma_{1}\right]$ verifies $\left(\Gamma_{2}^{2}\right)=\left(\Gamma_{1}^{2}\right)+1 ;$ indeed

$$
\left(\Gamma_{2}^{2}\right)=\left(g^{*}\left(\Gamma_{2}\right)^{2}\right)=\left(\Gamma_{1}+\mu L\right)^{2}=\left(\Gamma_{1}^{2}\right)+2 \mu\left(\Gamma_{1} . L\right)+\mu^{2}\left(L^{2}\right)=\left(\Gamma_{1}^{2}\right)+\mu^{2} ;
$$

(iii) any two curves meeting $L$ have intersecting images.

A useful consequence is the following:

CRITERION 7.3. When we blow down an exceptional curve $L=\sum L_{i}$ on a generalized Del Pezzo surface, the diagram of its irreducible roots and lines (as in 
§6) undergoes the following transformations:

a curve with self-intersection -2 (represented by a small circle) becomes exceptional (i.e. the small circle is replaced by a dot) if and only if it meets one and only one of the $L_{i}$;

on the other hand, it disappears from the picture if it meets two or more of the $L_{i}$;

an exceptional curve goes out of the picture if and only if it meets any of the $L_{i}$; nothing new can appear on the picture as long as we only blow down.

Armed with this criterion, we can now examine all fifteen cases of Proposition 5.6 and prove the following lemma:

LemMA 7.4. For $V \in \mathscr{V}_{0}$, the minimal disingularization $V^{\prime}$ contains an exceptional curve. In fact there exists a morphism $g: V^{\prime} \rightarrow V^{\prime \prime}$, which is obtained as a sequence of blowings-down, such that:

in Cases 2, 6, 7, 11, 12, and 15 of Proposition 5.6, $V^{\prime \prime}$ is isomorphic to $\mathbb{P}_{k}^{2}$;

in Cases $1,4,5,9,10$, and $14, V^{\prime \prime}$ is a form of $\mathbb{P}^{1} \times \mathbb{P}^{1}$;

in Cases 3, 8, and 13, $V^{\prime \prime}$ is a generalized Del Pezzo surface of degree 8, of type $A_{1}$, which is birationally equivalent to a form of $\mathbb{P}^{1} \times \mathbb{P}^{1}$.

Proof. For clarity we begin with Case 15; the reader should refer to the diagrams of $\S 6$.

15. There is a unique line; blow it down. By Criterion 7.3 we get a generalized Del Pezzo surface of degree 5, with diagram

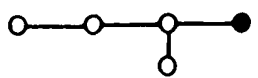

If we blow down the new line, we obtain

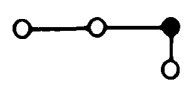

of degree 6. Another blowing down yields

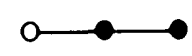

of degree 7. Now the middle dot corresponds to a line defined over $k$, which we can also blow down, thus obtaining $\bullet$, of degree 8 . This unique line can again be blown down, and the surface obtained is a Severi-Brauer surface, since it has degree 9 (cf. Proposition 0.4). As a matter of fact, this is a trivial Severi-Brauer variety, but we shall give a general argument further on.

14. Blowing down both lines, we get

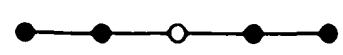

of degree 6. Blowing down the middle pair, we get a surface of degree 8 with no irreducible root and no exceptional curve, i.e. a form of $\mathbb{P}^{1} \times \mathbb{P}^{1}$.

13. Blowing down both lines, we get

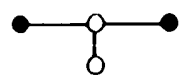


of degree 6. Blowing down the new pair of lines, we get $\circ$, that is, a surface of degree 8, of type $A_{1}$, which is a form of the ruled surface $F_{2}$.

The other cases are similar; so we shall deal with them more quickly. The resulting diagram is in brackets; the three possibilities are abbreviated $\mathbf{S B}, \mathbf{Q}$, and F respectively.

12. Blow down $l_{12}$ and $l_{5}(\bullet-\bullet)$. Blow down both lines (๑). Blow down once more (SB).

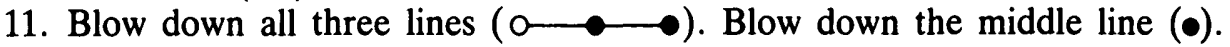
Blow down once more (SB).

10. Blow down $l_{3}$ and $l_{14}($ two roots $l_{0}-l_{1}-l_{2}-l_{3}$ and $l_{4}-l_{5}$, it is defined over $k$. So we can blow it down (Q).

9. Blow down all four lines $(\mathbf{Q})$.

8. Blow down all four lines $(\mathbf{F})$.

7. Blow down $l_{23}, l_{12}$, and $l_{5}(\longrightarrow)$. Blow down the ends (SB).

6. Blow down $l_{2}, l_{13}, l_{34}$, and $l_{5}(\bullet)$. Blow down once more (SB).

5. Blow down $l_{3}$ and $l_{24}\left(\right.$ Note that the root $l_{2}-l_{3}$ was defined over $k$. Since the other two roots give rise to the ends of the hexagon in brackets, we can blow down these two lines $(\mathbf{Q})$.

4. Blow down $l_{13}, l_{23}, l_{34}$, and $l_{5}(\mathbf{Q})$.

3. Since, by assumption, $V$ is not an Iskovskih surface, the two roots are individually defined over $k$. Thus we can blow down $l_{1}, l_{2}, l_{3}$, and $l_{45}(\mathbf{F})$.

2. Blow down $l_{24}, l_{3}, l_{5}, l_{12}$, and $l_{14}(\mathbf{S B})$.

1. Blow down $l_{5}, l_{14}, l_{24}$, and $l_{34}(\mathbf{Q})$.

Furthermore, the Severi-Brauer surface of Case SB is always trivial. Indeed it is obtained from a surface of degree 4 by blowing down 5 exceptional lines. Hence it contains a zero-cycle of degree 1 . Thus it is isomorphic to $\mathbb{P}_{k}^{2}$.

It remains to prove, in Case $\mathbf{F}$, that $V^{\prime \prime}$ is birationally equivalent to a form of $\mathbb{P}^{1} \times \mathbb{P}^{1}$. Now $V$, being a surface of degree 4 in $\mathbb{P}_{k}^{4}$, contains a Zariski-dense set of effective zero-cycles of degree 4 (it suffices to cut out $V$ by a family of $k$-rational planes). Hence we can find an effective 0 -cycle of degree 4 in general position on $V^{\prime \prime}$ and blow it up, thus obtaining a generalized Del Pezzo surface of degree 4 (cf. Proposition 0.4). Since there is only one root, we are in Case 1 of Proposition 5.6. But we proved above that a surface of this type is birationally equivalent to a form of $\mathbb{P}^{1} \times \mathbb{P}^{1}$.

We now proceed with the proof of the theorem.

(a) This follows from the more precise assertions of Lemma 7.4.

(b) Since the Clean Hasse Principle is birationallv invariant $[12, \S 3]$, it suffices to prove the usual Hasse principle for any smooth model of $V \in \mathscr{V}_{0}$. By Lemma 7.4 there exists a smooth model which is either $\mathbb{P}_{k}^{2}$ or a form of $\mathbb{P}^{1} \times \mathbb{P}^{1}$. The former case is trivial; in the latter case, the Hasse principle is well known to hold. (A very nice proof can be found in [9, pp. 19-20].)

However, the usual Hasse principle fails in general. Indeed the system (1) has solutions with $x_{0}=1$ over all completions of $\mathbb{Q}$, but no non-trivial rational solutions (just subtract the two equations!). The surface defined by (1), having four double points, is of Type 9. 
(c), (d) Again it suffices to prove these assertions for one smooth projective model. Now if $Z$ is a Severi-Brauer surface or a form of $\mathbb{P}^{1} \times \mathbb{P}^{1}$, they are well known to hold.

(e), (f) These follow from (d), as in Proposition 3.2.

(g) This follows trivially from (c).

Finally, none of the assertions of Theorem 7.2 holds for the class of Iskovskih surfaces. Indeed, a counter-example to $(\mathrm{c})-(\mathrm{g})$ is provided by the surface $V_{2}$ of Example 3.3, since we have already mentioned that in this case

$$
Z(\mathbb{Q}) / R=A_{0}(Z)=\uplus A_{0}(Z)=\uplus^{1}(\mathbb{Q}, S)=\mathbb{Z} / 2 .
$$

A counter-example to (b) is furnished by Iskovskih's example:

$$
\left\{\begin{array}{l}
x_{1}^{2}+x_{2}^{2}+x_{3}^{2}+x_{0} x_{3}=0 \\
3 x_{0}^{2}+x_{0} x_{3}-x_{4}^{2}=0 .
\end{array}\right.
$$

The singular points are $(0,1, \pm i, 0,0)$. This surface has smooth points everywhere locally, but no rational point [24]. This variety is also a counterexample to (a). Indeed the eight lines of the diagram of $\S 6$ can be computed explicitly. One sees that they form two orbits for the action of $\mathrm{Gal}(\overline{\mathbb{Q}} / \mathbb{Q})$ :

$$
\begin{aligned}
& x_{1} \pm i x_{2}=x_{4} \pm(\sqrt{ } 3) x_{0}=x_{3}=0 \\
& x_{1} \pm i x_{2}=x_{4} \pm(\sqrt{ } 2) x_{0}=x_{0}+x_{3}=0 .
\end{aligned}
$$

Thus $\operatorname{Gal}(\bar{Q} / \mathbb{Q})$, acting on this set, has no orbit consisting of skew lines. Hence there is no exceptional curve.

Alternatively, we may argue as follows. If the minimal desingularization $V^{\prime}$ of an Iskovskih surface is not a minimal surface, then there exists a birational morphism $g: V^{\prime} \rightarrow V^{\prime \prime}$, where $V^{\prime \prime}$ is a generalized Del Pezzo surface of degree $d \geqslant 5$. Consequently, assertions (b) and (c) of Corollary 9.4 hold for $V^{\prime \prime}$, and hence also for $V^{\prime}$ (cf. [29, Proposition 1.6]). Now it is known that any smooth model of $V_{2}$, respectively of the surface defined by equation (2), is a counterexample to (b), respectively to (c).

The next two propositions are analogues of Proposition 4.8 and Corollary 4.9, but here the existence of a $k$-point is not assumed (cf. Remark (a) after Corollary 4.9).

Proposition 7.5. Let $V \subset \mathbb{P}_{k}^{4}$ be an Iskovskih surface. Assume $(\operatorname{Pic} \bar{Z})^{G}=\operatorname{Pic} Z$, where $Z$ is any smooth projective model of $V$, and $\mathscr{G}=\operatorname{Gal}(\bar{k} / k)$. Then $V$ is $k$-birationally equivalent to a generalized Châtelet surface.

Proof. See [27, Theorem 4]. Alternatively, the assumption is equivalent to the condition $\mathrm{Br} k \hookrightarrow \mathrm{Br} k(V)$, via the standard exact sequence

$$
0 \rightarrow \operatorname{Pic} Z \rightarrow(\operatorname{Pic} \bar{Z})^{G} \rightarrow \operatorname{Br} k \rightarrow \operatorname{Br} Z .
$$

Now, the proposition under this condition is just Proposition 8(ii) of [15, Chapter IX].

PROPOSITION 7.6. Let $k$ be a number field, and $V \subset \mathbb{P}_{k}^{4}$ a singular intersection of two quadrics which has only isolated singularities and is not a cone. Let $Z$ be any smooth projective model of $V$. Then Manin's obstruction is the only obstruction to the Hasse principle for $Z$. 
Proof. By Proposition 0.3, $V$ has only rational double points. Hence the result follows from Theorem 7.2(b) if $V \in \mathscr{V}_{0}$. Indeed the Clean Hasse Principle, being birationally invariant, holds for $Z$ since it does for $V$.

Therefore we may assume that $V$ is an Iskovskih surface. Then we may apply Proposition 7.5. Indeed the assumption $Z\left(k_{v}\right) \neq \varnothing$ for all $v$ implies that $(\operatorname{Pic} \bar{Z})^{g}=$ Pic $Z$, as can be seen from the commutative diagram of exact sequences

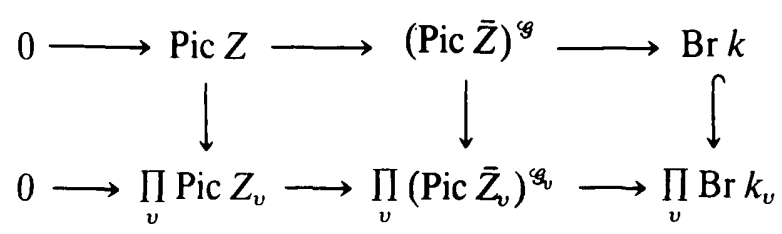

Thus $V$ is birationally equivalent to a generalized Châtelet surface. Hence the result follows from [15, Chapter VIII, Theorem 11].

Of course, many other properties can be obtained by combining Lemma 7.4 and Proposition 7.5 with the results of [15] on generalized Châtelet surfaces (cf. Corollary 4.9). The following result is also of interest:

Proposition 7.7. Let $V \subset \mathbb{P}_{k}^{4}$ be an Iskovskih surface. Suppose that the minimal desingularization $V^{\prime}$ of $V$ is a minimal surface. Then $V$ is not $k$-rational.

Proof. Using the diagram of $\S 6$ (Case 3), we describe a pencil of rational curves on $V^{\prime}$ : all four effective divisors $l_{1}+l_{14}, l_{2}+l_{24}, l_{3}+l_{34}$, and $l_{45}+l_{5}$, belong to the class $f=l_{0}-l_{4}$. Therefore $f$ lies in (Pic $\left.\bar{V}^{\prime}\right)^{G}$. We may clearly assume that $V(k) \neq \varnothing$, whence $f \in \operatorname{Pic} V^{\prime}$. As $\left(f^{2}\right)=0$ and $\left(f . \omega_{V^{\prime}}\right)=-2$, the curves in $f$ are rational and $h^{0}(f) \geqslant 2$. (This is the Riemann-Roch inequality, but in our case it is obvious, since we know four distinct effective divisors in the class!) Hence the effective divisors in the class $f$ describe a pencil of nonintersecting rational curves. (There is no superabundance, for $h^{0}(f)>2 \Rightarrow$ $\left(f^{2}\right) \neq 0$.) This shows that $V^{\prime}$ is a conic bundle surface with invariant $8-\left(\omega_{V^{\prime}}^{2}\right)=4$. By assumption $V^{\prime}$ is a minimal surface, and hence a relatively minimal conic bundle. Thus it follows from [25, Theorem 2], that $V^{\prime}$ is not birationally trivial over $k$.

Remark. The surfaces of degree 8 of type $A_{1}$ that occur in Lemma 7.4 include the minimal desingularizations of quadratic cones. Nevertheless, they seem to have been forgotten by almost everyone! As explained in the proof of Proposition 0.4, they were overlooked by Demazure (cf. [19, Proposition III.3(2.3)], in the case where $r=1$ ). Neither are these surfaces mentioned by Timms [44]. (But they do appear in Theorem 1.3 of [34].) A direct construction is as follows: take a point $x_{1}$ in $\mathbb{P}^{2}$, and an infinitely near point $x_{2}$ (i.e. a tangent direction at $x_{1}$ ); let $L$ be the line through $x_{1}$ in the direction $x_{2}$; blow up $x_{1}$ and then $x_{2}$; finally blow down the proper transform of $L$. This is analogous to the construction of smooth quadrics, except that $x_{2}$ is infinitely close to $x_{1}$. The image of $\mathbb{P}^{2}$ under the rational map defined by the linear system of conics through $x_{1}$ which are tangent to $x_{2}$, is a quadratic cone in $\mathbb{P}^{3}$. (If $x_{1}$ has coordinates $\left(X_{0}, X_{1}, X_{2}\right)=(1,0,0)$, and $x_{2}$ is in the direction $X_{2}=0$, then the map is defined by

$$
\left(X_{0}, X_{1}, X_{2}\right) \mapsto\left(X_{0} X_{2}, X_{1}^{2}, X_{1} X_{2}, X_{2}^{2}\right) \text {. }
$$

The equation of the image is $Y_{2}^{2}=Y_{1} Y_{3}$.) 


\section{Part III. SURfaces OF HIGHeR DEgReE}

\section{Classification of singularities}

This Part is devoted to a study of the singular Del Pezzo surfaces of degree $d \geqslant 5$. In this section we establish Proposition 0.8 in a stronger form, by proving, for each degree, the analogue of Proposition 5.6. Thus we may assume that $k$ is algebraically closed. Note, further, that $d \leqslant 8$. Indeed, as follows from Proposition 0.4, a generalized Del Pezzo surface of degree 9 contains no irreducible roots; so we can contract nothing. The reader will find it of some interest to compare the diagrams we obtain below with the pictures of Timms [44].

Proposition 8.1. Let $V$ be a singular Del Pezzo surface of degree $d=7$ or 8 . Then its minimal desingularization $V^{\prime}$ has exactly one irreducible root. More precisely,

if $d=8, V^{\prime}$ contains one irreducible root and no line:

if $d=7, V^{\prime}$ contains one irreducible root and two lines, with the following configuration:

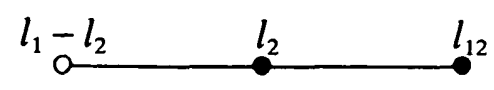

Proof. There is at least one irreducible root, since $V$ is singular. By Proposition 0.4 , this implies, for $d=8$, that $V^{\prime}$ is the ruled surface $F_{2}$. In this case the result follows from known properties of this surface (see [35, Proposition 2]). Alternatively, one may argue directly, using the fact that Pic $V^{\prime}$ is of rank $10-\left(\omega_{V^{\prime}}^{2}\right)=2$.

For $d=7, V^{\prime}$ is of the form $\mathbb{P}^{2}(\Sigma)$. So we can use the results of Demazure, in particular Proposition 5.5 and the analogue of Lemma 5.4. Up to sign, there is only one root, say $l_{1}-l_{2}$. Then the lines are determined as in $\S 6$, by means of Proposition 5.5.

Proposition 8.2. Let $V$ be a singular Del Pezzo surface of degree 6. Let $R_{i} \subset R$ be the set of irreducible roots on its minimal desingularization $V^{\prime}$. Then, up to the action of the Weyl group $W(R)$, the set $R_{i}$ belongs to one of the following types.

1. $l_{1}-l_{2}$.

2. $l_{0}-l_{1}-l_{2}-l_{3}$.

3. $l_{0}-l_{1}-l_{2}-l_{3}, l_{1}-l_{2}$.

4. $l_{1}-l_{2}, l_{2}-l_{3}$.

5. $l_{0}-l_{1}-l_{2}-l_{3}, l_{1}-l_{2}, l_{2}-l_{3}$.

Proof. The proof is on the same lines as that of Proposition 5.6. By the analogue of Lemma 5.4, we see that $\operatorname{Pic} V^{\prime} \simeq \mathbb{Z}^{4}$ and that $R$ is a root system of type $A_{1} \times A_{2}$ (apply [32, Chapter IV, Theorem 3.5]). We choose for simple roots the elements $\alpha=l_{0}-l_{1}-l_{2}-l_{3}, l_{1}-l_{2}$, and $l_{2}-l_{3}$ :

$$
\begin{aligned}
& \alpha \\
& \text { o } \\
& l_{1} \stackrel{\overbrace{}}{\stackrel{l_{2}}{\circ} l_{2}-l_{3}}
\end{aligned}
$$


Further, the Weyl group is known to be of the form

$$
W\left(A_{1}\right) \times W\left(A_{2}\right)=\mathscr{S}_{2} \times \mathscr{S}_{3} .
$$

The generator of $\mathscr{S}_{2}$ replaces $\alpha$ by its opposite, and $\mathscr{S}_{3}$ acts by permuting the $l_{i}$ $(i \neq 0)$. Thus the proof reduces to the following two independent assertions.

(i) Up to the action of $W\left(A_{1}\right)$, there are two possibilities: if $\alpha$ is not an irreducible root, then we may assume that neither $\alpha$ nor $-\alpha$ is irreducible.

(ii) Up to the action of $W\left(A_{2}\right)$, the other irreducible roots form one of the following sets:

$$
\varnothing, \quad\left\{l_{1}-l_{2}\right\}, \quad\left\{l_{1}-l_{2}, l_{2}-l_{3}\right\} .
$$

These assertions are proved quite easily. This yields $2.3=6$ different types, including the case in which $R_{i}$ is empty. This case is not mentioned in the statement, since $V$ is assumed to be singular.

As in $\S 6$, this proposition can be restated as follows:

Proposition 8.3. The configuration of irreducible roots and lines on the minimal desingularization of a singular Del Pezzo surface of degree 6 is described by one of the following diagrams:

1. (4 lines)

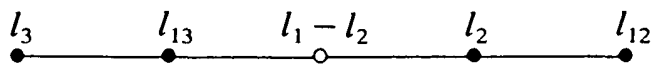

2. (3 lines)

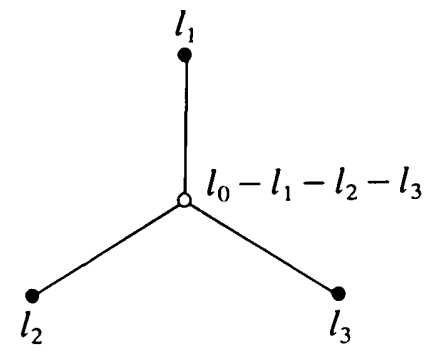

3. (2 lines)

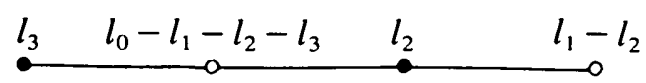

4. (2 lines)

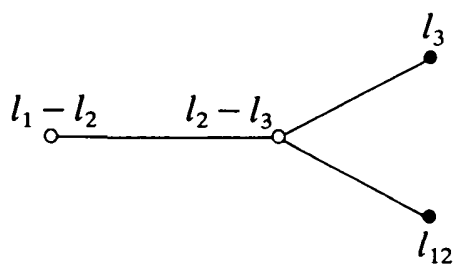


5. (1 line)

$$
\begin{array}{llll}
l_{1}-l_{2} & l_{2}-l_{3} & l_{3} & l_{0}-l_{1}-l_{2}-l_{3}
\end{array}
$$

Proposition 8.4. Let $V$ be a singular Del Pezzo surface of degree 5. Let $R_{i} \subset R$ be the set of irreducible roots on its minimal desingularization $V^{\prime}$. Then, up to the action of the Weyl group $W(R)$, the set $R_{i}$ belongs to one of the following types.

1. $l_{1}-l_{2}$.

2. $l_{1}-l_{2}, l_{3}-l_{4}$.

3. $l_{1}-l_{2}, l_{2}-l_{3}$.

4. $l_{1}-l_{2}, l_{2}-l_{3}, l_{0}-l_{1}-l_{2}-l_{3}$.

5. $l_{1}-l_{2}, l_{2}-l_{3}, l_{3}-l_{4}$.

6. $l_{1}-l_{2}, l_{2}-l_{3}, l_{3}-l_{4}, l_{0}-l_{1}-l_{2}-l_{3}$.

Proof. By the analogue of Lemma 5.4, we see that Pic $V^{\prime} \simeq \mathbb{Z}^{5}$ and that $R$ is a root system of type $A_{4}$ (apply [32, Chapter IV, Theorem 3.5]). We use the standard realization of this system in $\mathbb{R}^{5}$, as in [5, Chapter VI, $\S 4$, no. 7]. Note that, by Proposition 1 of [5, Chapter VI, $\S 4$, no. 2], we may identify

$$
\varepsilon_{1}-\varepsilon_{2}, \quad \varepsilon_{2}-\varepsilon_{3}, \quad \varepsilon_{3}-\varepsilon_{4}, \quad \text { and } \varepsilon_{4}-\varepsilon_{5}
$$

with

$$
l_{1}-l_{2}, \quad l_{2}-l_{3}, \quad l_{3}-l_{4}, \quad \text { and } \quad l_{0}-l_{1}-l_{2}-l_{3},
$$

in this order. Since we know that $W\left(A_{4}\right) \simeq \mathscr{S}_{5}$ acts by permuting the base vectors $\varepsilon_{i}$, the proof now proceeds exactly as in Proposition 5.6.

Proposition 8.5. The configuration of irreducible roots and lines on the minimal desingularization of a singular Del Pezzo surface of degree 5 is described by one of the following diagrams.

1. (7 lines)

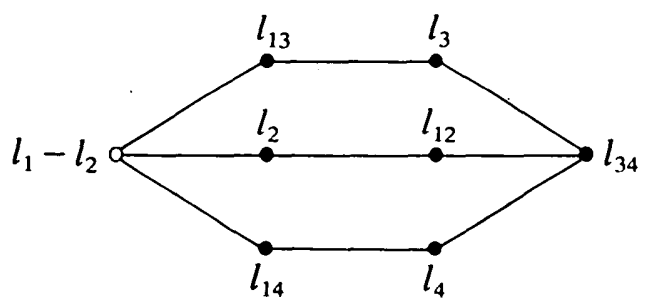

2. (5 lines)

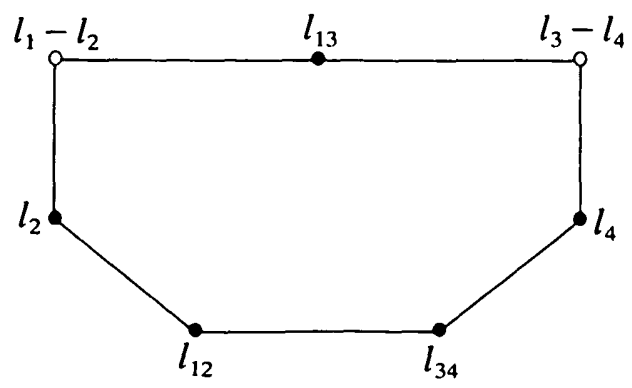


3. (4 lines)

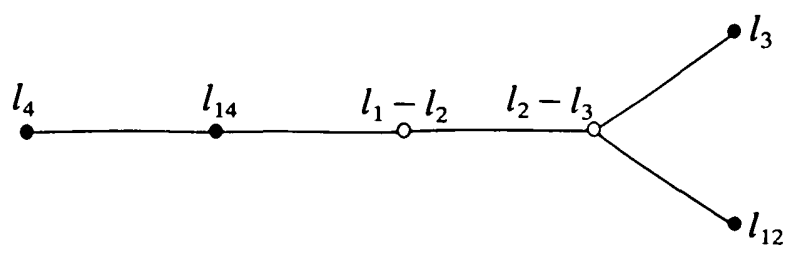

4. (3 lines)

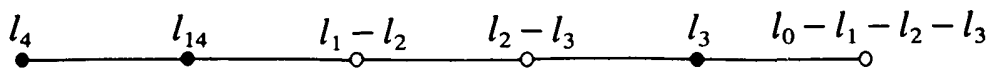

5. (2 lines)

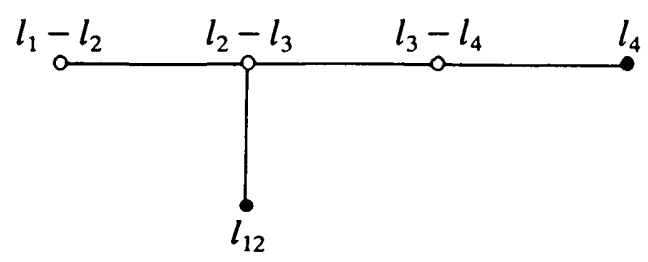

6. (1 line)

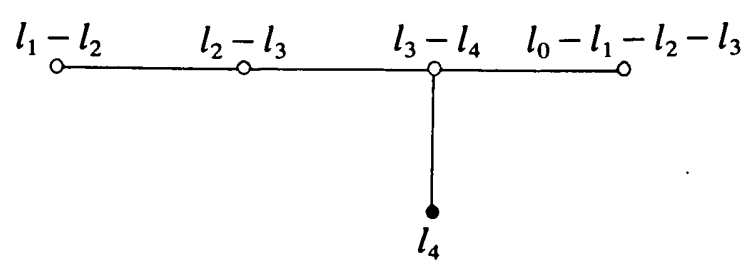

Proposition 8.6. All the cases described occur; that is, for each one of the sets $R_{i}$ listed in Propositions 8.1, 8.2, 8.4, and 5.6, there exists a singular Del Pezzo surface $V$ of the corresponding degree whose set of irreducible roots in Pic $V^{\prime}$ is the given set $R_{i}$.

Sketch of proof. It suffices to construct a generalized Del Pezzo surface $V^{\prime}$ with the prescribed set of irreducible roots $R_{i}$. Since we have a complete classification for all degrees, we can proceed by induction. Thus in most cases a surface of degree $d$ is obtained from a surface of degree $d+1$ by blowing up only one point. By Proposition 0.4 we know that it is enough to make sure that this point does not lie on any irreducible root, and this condition is easy to check on the diagrams. We omit the details (cf. $[44, \S 8]$ ).

\section{Arithmetical applications}

In this section, $k$ denotes an arbitrary perfect field. We use the classification of singularities over $\bar{k}$ to establish the following result (cf. Theorem 7.2). 
THEOREM 9.1. Let $\mathscr{V}_{d}$ be the family of all singular Del Pezzo surfaces of degree $d$.

(a) For $d=5,6$, or 7 , the minimal desingularization $V^{\prime}$ of any variety $V \in \mathscr{V}_{d}$ is a non-minimal surface.

(b) If $d=5$ or 7 , then any surface $V \in \mathscr{V}_{d}$ is $k$-rational.

(c) If $d \geqslant 5$, any surface $V \in \mathscr{V}_{d}$ with a smooth $k$-point is $k$-rational.

(d) Let $Z$ be any smooth projective model of a variety $V \in \mathscr{V}_{d}$, where $d \geqslant 5$. Then $A_{0}(Z)=0$.

(e) If $k$ is a number field and $d \geqslant 5$, then the 'Clean Hasse Principle' holds for the class $\mathscr{V}_{d}$ : if $V \in \mathscr{V}_{d}$ contains a smooth point defined over $k_{v}$, for every completion $k_{v}$ of $k$, then every proper model of $V$ contains a $k$-point.

Proof. To begin with, we consider the case of degree 8 , for which a special argument is required.

Lemma 9.2. Let $V$ be a singular Del Pezzo surface of degree 8. We denote by $B$ the irreducible root on its minimal desingularization $V^{\prime}$.

(i) The variety obtained from $V^{\prime}$ by blowing up a point not lying on $B$ is a generalized Del Pezzo surface of degree 7 (with an irreducible root).

(ii) If $B(k) \neq \varnothing$, then $V^{\prime}$ also contains a $k$-point that does not lie on $B$.

(iii) If $k$ is a number field, then the Hasse principle holds for $V^{\prime}$.

Proof. As we saw in the proof of Proposition 8.1, $\bar{V}^{\prime}$ is the ruled surface $F_{2}$. Recall $[35, \S 2]$ that this surface contains a unique irreducible curve with negative self-intersection, which is called the base line. (This is $B$, of course; and it is smooth.) Furthermore, it contains a unique pencil of non-intersecting rational curves, called the fibres, all of which are irreducible and smooth. If $F$ denotes any fibre, the following relations hold:

$$
\left(F^{2}\right)=0, \quad(B . F)=1, \quad\left(B^{2}\right)=-2 .
$$

Now we can proceed with the argument.

(i) If $P$ is a point of $V^{\prime}$ that does not lie on the base line $B$, it is known [35, Corollary to Proposition 1] that the elementary transformation centred at $P$ carries $\bar{V}^{\prime}$ into the ruled surface $F_{1}$, which is $\mathbb{P}^{2}$ with a point blown up. Therefore blowing up $P$ on $\bar{V}^{\prime}$ yields a surface of the form $\mathbb{P}^{2}(\Sigma)$, namely $F_{1}$ with a point blown up, whence the result follows.

(ii) If $P \in B(k)$, let $F$ be the fibre through $P$. It is uniquely determined, since $\left(F^{2}\right)=0$. Thus $F$ is defined over $k$, whence it is isomorphic to $\mathbb{P}_{k}^{1}$. Therefore we can find on $F$ a $k$-point that does not lie on $B$.

(iii) If $P \in V^{\prime}\left(k_{v}\right)$, let $F$ be the fibre through $P$. Since $(B . F)=1$, the intersection of $F$ with $B$ is a $k_{v}$-point. Thus $B\left(k_{v}\right) \neq \varnothing$. Now $B$, being a smooth rational curve, verifies the Hasse principle. Hence we have shown that

$$
\begin{aligned}
V^{\prime}\left(k_{v}\right) \neq \varnothing \text { for all } v & \Rightarrow B\left(k_{v}\right) \neq \varnothing \text { for all } v \\
& \Rightarrow B(k) \neq \varnothing \\
& \Rightarrow V^{\prime}(k) \neq \varnothing .
\end{aligned}
$$

Remark. A cone over a non-trivial conic provides an example of degree 8 where the word 'smooth' cannot be deleted in Theorem 9.1(c). This creates the interest of the second assertion in Lemma 9.2. (Cf. the proof of Corollary 9.4.) 
LEMMA 9.3. For $d=5,6$, or 7, the minimal desingularization $V^{\prime}$ of any variety $V \in \mathscr{V}_{d}$ contains an exceptional curve. In fact there exists a morphism $g: V^{\prime} \rightarrow V^{\prime \prime}$, which is obtained as a sequence of blowings-down, such that $V^{\prime \prime}$ is isomorphic to $\mathbb{P}_{k}^{2}$, except in Cases 1 and 4 of Proposition $8.3(d=6)$, where $V^{\prime \prime}$ is a form. of the ruled surface $F_{2}$. (In Case $1, V^{\prime \prime}$ may also be chosen to be a form of $\mathbb{P}^{1} \times \mathbb{P}^{1}$.)

Proof. As in the proof of Lemma 7.4, we apply Criterion 7.3 successively to the diagrams of Propositions 8.1, 8.3, and 8.5. The details are easy, but there are a few interesting cases.

(a) $d=7$. Begin by blowing down $l_{2}$ (not $l_{12}$, which leads to a form of $F_{2}$ !).

(b) $d=6$. 1. Blow down $l_{3}$ and $l_{12}$ to get a form of $F_{2}$. (By blowing down $l_{2}$ and $l_{13}$, we obtain a form of $\mathbb{P}^{1} \times \mathbb{P}^{1}$.)

2. By blowing down $l_{1}, l_{2}$, and $l_{3}$, we get a Severi-Brauer surface. To see that it is $\mathbb{P}_{k}^{2}$, note that the proper transform of the irreducible root is a smooth rational curve with self-intersection 1 . Hence it is isomorphic to $\mathbb{P}_{k}^{1}$, and the SeveriBrauer variety is trivial.

3 and 5. By blowing down $l_{3}$, we are reduced to the case of degree 7 .

4. Blow down $l_{3}$ and $l_{12}$.

(c) $d=5$. 1. By blowing down $l_{34}$, we are reduced to Case 2 of degree 6 .

2. Do not blow down $l_{13}$ ! Blow down $l_{2}$ and $l_{4}$. This yields a smooth Del Pezzo surface of degree 7 (—). Blow down the ends, so as to obtain a trivial Severi-Brauer surface (since $l_{13}$ is defined over $k$ ).

3. By blowing down $l_{3}$ and $l_{12}$, we are reduced to the case of degree 7 . (It would be a bad idea to blow down $l_{4}$.)

4 and 6 . By blowing down $l_{4}$, we are reduced to Case 5 of degree 6 .

5. By blowing down $l_{12}$, we are reduced to Case 3 of degree 6 .

Now it is easy to complete the proof of Theorem 9.1 . For $d=5$ or 7 , all the assertions follows trivially from Lemma 9.3 (where $V^{\prime \prime} \simeq \mathbb{P}_{k}^{2}$ ).

This lemma also implies assertion (a) for $d=6$. Now we remember that both assertions (d) and (e) are birationally invariant (cf. [11, Proposition 6.3; 12, § 3]). Hence, by another application of Lemma 9.3, it is enough to prove (c)-(e) for a form of the ruled surface $F_{2}$. Of course this will also settle the case where $d=8$, since a form of $F_{2}$ is nothing but the minimal desingularization $V^{\prime}$ of a singular Del Pezzo surface of degree 8.

We therefore appeal to Lemma 9.2. Assertion (e) for $V^{\prime}$ is already contained in (iii). And assertion (c) follows from (i) and (ii), since we have just seen that a generalized Del Pezzo surface of degree 7 is $k$-rational. Finally, we compute $A_{0}\left(V^{\prime}\right)$. Clearly, in view of (c), we may assume that $V^{\prime}(k)=\varnothing$. Further, as we saw in the proof of Lemma 9.2, we can view $V^{\prime}$ as a conic bundle surface $\varphi: V^{\prime} \rightarrow B$ over the base $B$, which is actually contained in $V^{\prime}$. Hence, by Lemma 3.7 of [11], every effective 0 -cycle $A$ on $V^{\prime}$ is rationally equivalent to an effective 0 -cycle of the same degree, $C=\varphi_{*}(A)$, contained in $B$. Since $B$ is a smooth rational curve, we can fix an effective 0 -cycle $C_{0}$ of degree 2 on $B$, and then every 0 -cycle on $V^{\prime}$ is rationally equivalent to some multiple of $C_{0}$. Hence $A_{0}\left(V^{\prime}\right)=0$.

CoRollary 9.4. Let $\mathcal{W}_{d}$ be the family of all generalized Del Pezzo surfaces of degree $d$.

(a) If $d=5$ or 7 , then any surface $W \in W_{d}$ is $k$-rational.

(b) If $d \geqslant 5$, any surface $W \in W_{d}$ with a $k$-point is $k$-rational. 
(c) If $k$ is a number field and $d \geqslant 5$, then the Hasse principle holds for the class $W_{d}$.

Proof. These results are known to hold for the class of smooth Del Pezzo surfaces (cf. [32, Chapter IV]). Thus we may assume that $W \in \mathcal{W}_{d}$ is the minimal desingularization of a singular Del Pezzo surface $V$ of degree $d$, and apply Theorem 9.1. There is one minor difficulty: for proving assertion (b), one must know that $V$ has a smooth $k$-point if $W(k) \neq \varnothing$. But the problem arises only for $d=6$ or 8 . By Lemma 9.3 , it suffices to consider the latter case. But, for $d=8$, the difficulty is resolved by the second assertion of Lemma 9.2.

\section{References}

1. M. ARTIN, 'Some numerical criteria for contractability of curves on algebraic surfaces', Amer. J. Math. 84 (1962) 485-496.

2. M. ARTIN, 'On isolated rational singularities of surfaces', Amer. J. Math. 88 (1966) 129-136.

3. A. Beauville, J-L. Colliot-Thélène, J-J. Sansuc, and Sir P. Swinnerton-Dyer, 'Variétés stablement rationnelles non rationnelles', Ann. of Math. 121 (1985) 283-318.

4. E. BOMBIERI and D. HUSEMOLLER, 'Classification and embeddings of surfaces', Proceedings of Symposia in Pure Mathematics 29 (American Mathematical Society, Providence, R.I., 1975), pp. 329-420.

5. N. BouRBAKI, Groupes et algebres de Lie (Hermann, Paris, 1968), Chapters 4-6.

6. J. W. BRUCE and C. T. C. WALL, 'On the classification of cubic surfaces', J. London Math. Soc. (2) 19 (1979) 245-256.

7. J. BRZEZIŃSKI, 'Arithmetical quadratic surfaces of genus 0, I', Math. Scand. 46 (1980) 183-208.

8. A. Cayley, 'A memoir on cubic surfaces', Philos. Trans. Roy. Soc. London 159 (1869) 231-326; Collected Papers VI (Cambridge University Press, 1893), pp. 359-455.

9. J-L. Colliot-ThÉlène, 'Quelques propriétés arithmétiques des surfaces rationnelles', Sém. de Théorie des Nombres, Bordeaux (1971-72), exposé 13, 22pp.

10. J-L. Colliot-ThÉlÈnE, 'Hilbert's Theorem 90 for $K_{2}$, with application to the Chow groups of rational surfaces', Invent. Math. 71 (1983) 1-20.

11. J-L. COLLIOT-THÉLĖNE and D. F. CORAY, 'L'équivalence rationnelle sur les points fermés des surfaces rationnelles fibrées en coniques', Compositio Math. 39 (1979) 301-332.

12. J-L. Colliot-Thélène, D. Coray, and J-J. Sansuc, 'Descente et principe de Hasse pour certaines variétés rationnelles', J. reine angew. Math. 320 (1980) 150-191.

13. J-L. Colliot-ThÉLĖNE and J-J. SANSUC, 'La descente sur les variétés rationnelles', Journées de géométrie algébrique d'Angers, 1979 (Sijthoff and Noordhoff, Alpen aan den Rijn, 1980), pp. 223-237.

14. J-L. Colliot-ThélÈne and J-J. SANSUC, 'On the Chow groups of certain rational surfaces: a sequel to a paper of S. Bloch', Duke Math. J. 48 (1981) 421-447.

15. J-L. Colliot-Thélène, J-J. SANSUC, and Sir P. Swinnerton-Dyer, 'Intersections of two quadrics and Châtelet surfaces', J. reine angew. Math. 373 (1987) 37-107, 374 (1987) 72-168.

16. K. R. CoOmbes and D. J. Muder, 'Zero-cycles on Del Pezzo surfaces over local fields', J. Algebra 97 (1985) 438-460.

17. D. F. CORAY, 'Arithmetic on singular cubic surfaces', Compositio Math. 33 (1976) 55-67.

18. H. S. M. COXETER, 'Finite groups generated by reflections, and their subgroups generated by reflections', Proc. Cambridge Philos. Soc. 30 (1934) 466-482.

19. M. Demazure, 'Surfaces de Del Pezzo', Séminaire sur les singularités des surfaces, Lecture Notes in Mathematics 777 (Springer, Berlin, 1980), pp. 21-69.

20. P. Du VAL, 'On isolated singularities of surfaces which do not affect the conditions of adjunction' (Parts II and III), Proc. Cambridge Philos. Soc. 30 (1934) 460-465, 483-491.

21. R. HARTSHORNE, Ample subvarieties of algebraic varieties, Lecture Notes in Mathematics 156 (Springer, Berlin, 1970).

22. H. P. HUDSON, Cremona transformations (Cambridge University Press, 1927).

23. V. A. IsKovSKIH, 'Rational surfaces with a pencil of rational curves and with positive square of the canonical class', Mat. Sb. 83 (1970) 90-119; Math. USSR-Sb. 12 (1970) 91-117.

24. V. A. IsKovsKiH, 'A counterexample to the Hasse principle for a system of two quadratic forms in five variables', Mat. Zametki 10 (1971) 253-257; Math. Notes 10 (1971) 575-577.

25. V. A. IsKovSKIH, 'Birational properties of a surface of degree 4 in $\mathbb{P}_{k}^{4}$ 'Mat. Sb. 88 (1972) 31-37; Math. USSR-Sb. 17 (1972) 30-36. 
26. H. KNÖRRER, Isolierte Singularitäten von Durchschnitten zweier Quadriken, dissertation, Bonner Mathematische Schriften 117, 1980, 132pp.

27. B. Ė. Kunjavskiì, A. N. Skorobogatov, and M. A. Tsfasman, 'Combinatorics and geometry of Del Pezzo surfaces of degree 4', Uspekhi Mat. Nauk 40:6 (1985) 145-146; Russian Math. Surveys 40 (1985) 131-132.

28. B. Ė. Kunjavskil, A. N. Skorobogatov, and M. A. Tsfasman, 'Del Pezzo surfaces of degree 4', Bull. Soc. Math. France, to appear.

29. B. E. KunjavSKiI and M. A. TsfaSman, 'Zero-cycles on rational surfaces, and Néron-Severi tori', Izv. Akad. Nauk SSSR 48 (1984) 631-654; Math. USSR-Izv. 24 (1985) 583-603.

30. J. LIPMAN, 'Rational singularities, with applications to algebraic surfaces and unique factorization', Publications Mathématiques 36 (Institut des Hautes Études Scientifiques, Paris, 1969), pp. 195-279.

31. E. J. N. LOOIJENGA, Isolated singular points on complete intersections, London Mathematical Society Lecture Note Series 77 (Cambridge University Press, 1984).

32. Ju. I. Manin, Cubic forms (Nauka, Moscow, 1972; North-Holland, Amsterdam, 1974).

33. Ju. I. MANIN and M. A. TSFaSman, 'Rational varieties: algebra, geometry, arithmetic', Uspekhi Mat. Nauk 41:2 (1986) 43-94; Russian Math. Surveys 41 (1986) 51-116.

34. J-Y. MÉRINDOL, 'Les singularités simples elliptiques, leurs déformations, les surfaces de Del Pezzo et les transformations quadratiques', Ann. Sci. École Norm. Sup. 15 (1982) 17-44.

35. M. Nagata, 'On rational surfaces I', Mem. Coll. Sci. Univ. Kyoto ser. A 32 (1960) 351-370.

36. I. NARUKI and T. URABE, 'On degenerate Del Pezzo surfaces of degree 1,2', preprint (announced in Proceedings of Symposia in Pure Mathematics 40 (American Mathematical Society, Providence, R.I., 1983), pp. 587-591).

37. H. C. Pinkham, 'Simple elliptic singularities, Del Pezzo surfaces and Cremona transformations', Proceedings of Symposia in Pure Mathematics 30 (American Mathematical Society, Providence, R.I., 1977), pp. 69-71.

38. J-J. SANSUC, 'A propos d'une conjecture arithmétique sur le groupe de Chow d'une surface rationnelle', Sém. de Théorie des Nombres, Bordeaux (1981-82), exposé 33, 38 pp.

39. L. SCHLÄFL, 'On the distribution of surfaces of the third order into species, in reference to the absence or presence of singular points, and the reality of their lines', Philos. Trans. Roy. Soc. London 153 (1863) 193-241; Gesammelte Abhandlungen, II (Birkhäuser, Basel, 1953), pp. 304-362.

40. B. SEGRE, 'On arithmetical properties of singular cubic surfaces', J. London Math. Soc. 19 (1944) 84-91.

41. TH. SKolem, 'Einige Bemerkungen über die Auffindung der rationalen Punkte auf gewissen algebraischen Gebilden', Math. Z. 63 (1955) 295-312.

42. H. P. F. SWINNERTON-DYer, 'The zeta function of a cubic surface over a finite field', Proc. Cambridge Philos. Soc. 63 (1967) 55-71.

43. H. P. F. SWINNERTON-DYer, 'The birationality of cubic surfaces over a given field', Michigan Math. J. 17 (1970) 289-295.

44. G. TIMms, 'The nodal cubic surfaces and the surfaces from which they are derived by projection', Proc. Roy. Soc. London Ser. A 119 (1928) 213-248.

45. M. A. TSFASMAN, 'Rational points on algebraic curves and surfaces' (in Russian), dissertation, Moscow State University, Moscow, 1982, $100 \mathrm{pp}$.

46. V. E. VosKreSENSKII, Algebraic tori (in Russian, Nauka, Moscow, 1977).

47. C. T. C. WALL, 'The first canonical stratum', J. London Math. Soc. (2) 21 (1980) 419-433.

Section de Mathématiques

Université de Genève

2-4, rue du Lièvre

CH-1211 Genève 24

Switzerland
10-75, ul.Udal'tsova

Moscow 117415

U.S.S.R. 